\author{
Universidade de São Paulo \\ Instituto de Física
}

\title{
Estados emaranhados quânticos tri-partidos com um qubit
}

\author{
Marcio Fernando Cornelio
}

Orientador: Prof. Dr. Antonio Fernando Ribeiro de Toledo Piza

Tese de doutorado submetida

ao Instituto de Física

para a obtenção do título

de Doutor em Ciências

Comissão examinadora:

Prof. Dr. Antonio Fernando Ribeiro de Toledo Piza (USP)

Prof. Dr. Arnaldo Gammal (USP)

Prof. Dr. Marcos Cesar de Oliveira (Unicamp)

Prof. Dr. Paulo Alberto Nussenzveig (USP)

Prof. Dr. Roberto Menezes Serra (UFABC)

São Paulo 


\section{FICHA CATALOGRÁFICA}

Preparada pelo Serviço de Biblioteca e Informação do Instituto de Física da Universidade de São Paulo

\begin{tabular}{|c|}
\hline Cornelio, Marcio Fernando \\
Estados emaranhados quânticos tri-partidos com um \\
qubit - 2008 \\
Tese (Doutorado) - Universidade de São Paulo. \\
Instituto de Física - Depto. de Física Matemática \\
\hline Orientador: Prof. Dr. Antonio Fernando Ribeiro de \\
\hline Toledo Piza \\
Área de Concentração: Física \\
\hline Unitermos: 1. Física; 2. Mecânica Quântica; \\
3.Informação Quântica. \\
USP/IF/SBI-041/2008
\end{tabular}


a meus pais 



\section{Resumo}

Estudamos o emaranhamento quântico de estados puros emaranhados tri-partidos quando uma das partes é um qubit. Apresentamos um método para encontrar as decomposições do estado tri-partido mais simples do que sucessivas decomposições de Schmidt. Esse método permite encontrar uma grande quantidade de diferentes famílias de estados emaranhados tri-partidos. Essas famílias são classificadas de acordo com dimensão dos blocos de Jordan de uma matriz obtida do estado emaranhado. Além disso, também demonstramos que estados pertencentes a famílias distintas não podem ser convertidos um no outro por operações locais estocásticas com comunicação clássica (SLOCC). No caso de dois estados pertencentes à mesma família, obtemos condições necessárias e suficientes para sabermos se estes podem ser convertidos um no outro por SLOCC. No caso de serão, também podemos obter a operação do tipo SLOCC que realiza a conversão. 



\begin{abstract}
We study the quantum entanglement of tripartite pure states when one of the parties is a qubit. We present a method to find the decompositions of tripartite entangled states which are simpler than two successive Schmidt decompositions. We will find many distinct families of entangled states with distinct decompositions. These families are classified according to the dimensions of the Jordan blocks of a matrix obtained from the entangled state. Furthermore, we show that states belonging to distinct families can not be converted into each other by stochastic local operations and classical communication (SLOCC). In case of two states belonging to the same family, we find necessary and sufficient conditions to convert one state to the other. We can also find the SLOCC which realizes this conversion.
\end{abstract}





\section{Sumário}

Agradecimentos $\quad$ xi

$\begin{array}{ll}\text { Introdução } & 1\end{array}$

1 O emaranhamento quântico 3

1.1 Estados emaranhados puros bi-partidos . . . . . . . . . . . . . . 3

1.1.1 O que são estados emaranhados? . . . . . . . . . . . . . 3

1.1.2 Correlações Quânticas em estados emaranhados: estados relativos . . 8

1.1.3 O estado dos subsistemas . . . . . . . . . . . . . . 9

1.1.4 A Representação de Schmidt ou bi-ortogonal . . . . . . . . . . . . . 11

1.1.5 Quantificação do emaranhamento . . . . . . . . . . . . . 13

1.2 Estados emaranhados multi-partidos . . . . . . . . . . . . . . . . . 14

1.3 Informação quântica e emaranhamento . . . . . . . . . . . . . . . . . . . . 17

1.3.1 Código denso . . . . . . . . . . . . . . . . . . . . . . . . 18

1.3.2 Teletransporte quântico . . . . . . . . . . . . . . . . . . 19

2 Transformações locais dos estados emaranhados $\quad 21$

2.1 Transformações unitárias locais . . . . . . . . . . . . . . . . 21

2.2 Operações locais estocásticas com comunicação clássica . . . . . . . . . . . . 22

2.2 .1 Operações Quânticas . . . . . . . . . . . . . . . . . . 23

2.2.2 Exemplos com dois qubits . . . . . . . . . . . . . . 26

2.2.3 Critério de convertibilidade para SLOCC . . . . . . . . . . . 29

2.2.4 Exemplo: Classificação SLOCC para o caso bi-partido . . . . . . . . 34

2.2.5 Operações SLOCC e sistemas multi-partidos . . . . . . . . . . . 35

$\begin{array}{lll}3 & \text { Emaranhamento tri-partido com um qubit } & \mathbf{3 7}\end{array}$

3.1 Decomposições de estados emaranhados com um qubit . . . . . . . . . . . . . 38

3.2 Exemplos . . . . . . . . . . . . . . . . . . . . . 45

3.2.1 Exemplos: Três qubits. . . . . . . . . . . . . . . . . 45

3.2.2 Exemplos: dois sistemas de 3 níveis e um qubit . . . . . . . . . . 47 
3.2.3 Exemplos: dois subsistemas de quatro níveis e um qubit . . . . . . . 54

3.2 .4 Contra-exemplos . . . . . . . . . . . . . . . 76

3.3 Decomposições sub-Schmidt e operações do tipo SLOCC . . . . . . . . . . . . 78

3.4 Discussão: Estados emaranhados tri-partidos mais gerais . . . . . . . . . . 82

$\begin{array}{ll}\text { Conclusão } & 85\end{array}$

$\begin{array}{lr}\text { Referências Bibliográficas } & 87\end{array}$ 


\section{Agradecimentos}

Primeiramente, gostaria de agradecer a meus pais, Luiz Fernando Cornelio e Vanice Maria Cornelio, pelo grande apoio e confiança que sempre depositaram em mim. Gostaria de agradecer ainda meus colegas do Dep. de Física Matemática dentre os quais fiz grandes amigos.

Gostaria de agradecer também ao Prof. Dr. Antonio Fernando Ribeiro de Toledo Piza pela orientação, pelos conselhos e sugestões sempre bastante relevantes. Não posso deixar de agradecer também ao Prof. Dr. Daniel Tausk pela ajuda na demonstração do Teorema 3.1 e ao Dr. Jacob Dunningham e ao Prof. Dr. Vlatko Vedral pela supervisão do meu estágio na Universidade de Leeds.

Por fim, gostaria de agradecer à FAPESP (Fundação de Amparo a Pesquisa do Estado de São Paulo) pelo suporte financeiro e também ao IFUSP (Instituto de Física da Universidade de São Paulo) pelo usufruto das excelentes instalações e recurso. 



\section{Introdução}

Atualmente, o interesse em informação quântica tem motivado fortemente o estudo do emaranhamento quântico. Embora o emaranhamento quântico multi-partido ainda seja pouco conhecido, não há dúvidas sobre a sua fundamental importância para a informação quântica. Isso deve-se principalmente a algumas aplicações simples dos estados emaranhados bi-partidos a tarefas de informação quântica como o código denso [1], o teletransporte quântico [2] e a distribuição de chave secreta [3]. Além disso, a coerência de um estado emaranhado multi-partido é fundamental para a implementação de algoritmos quânticos como os algoritmos de Shor e de Grover $[4,5]$. Nesse sentido, um melhor entendimento do emaranhamento quântico é algo bastante desejável.

Para atingirmos tal objetivo, primeiro gostaríamos de observar que o entendimento atual dos estados emaranhados puros de sistemas compostos bi-partidos é bastante embasado na decomposição de Schmidt [6] e também na decomposição em estados relativos $[7,8,9]$. Essas representações são centrais no desenvolvimento de diversos trabalhos fundamentais em mecânica quântica tais como a teoria de medição de von Neumann [10], o argumento de incompletude de Einstein, Podolski e Rosen [11] e o problema do gato de Schrödinger $[7,8,12]$. Hoje em dia, essas decomposições têm sido bastante utilizadas em vários resultados da teoria do emaranhamento bi-partido para estados puros [13, 14, 15]. Uma propriedade importante dessas decomposições é a sua simplicidade, pois o estado emaranhado é escrito com o menor número possível de estados produto.

Para sistemas multi-partidos, poderíamos utilizar essas decomposições recursivamente fazendo sucessivas bi-partições do sistema composto [16]. No caso de um sistema composto de três qubits, por exemplo, conseguiríamos sempre obter uma decomposição em no máximo quatro estados produto. No entanto, Dür et al [17] demonstraram que sempre existem decomposições mais simples com dois ou três estados produto.

Além disso, em geral, as aplicações do emaranhamento quântico à informação quântica estão relacionadas aos aspectos não locais dos estados emaranhados. Nesses casos, nos quais devemos considerar que os subsistemas estão espacialmente separados, é importante sabermos em quais estados um determinado estado emaranhado pode ser transformado por operações locais. Nesse sentido, Dür et al [17] também demonstraram que as decomposições 
mais simples, isto é, no menor número de estados produto, são úteis para entendermos as propriedades dos estados emaranhados multi-partidos com relação às operações locais estocásticas com comunicação clássica (SLOCC) [18].

No entanto, não existe nenhum método para encontrar tais decomposições e isso dificulta bastante o entendimento dos estados emaranhados multi-partidos. Nesse sentido, desenvolveremos um método capaz de encontrar essas decomposições para certos estados emaranhados tri-partidos sendo que um dos subsistemas é um qubit. As decomposições encontradas serão úteis para obtermos algumas propriedades desses estados bem como estabelecer quais deles são equivalentes por operações locais do tipo SLOCC.

Esta tese é organizada da seguinte maneira. No Capítulo 1, introduzimos o conceito de emaranhamento quântico e descrevemos em detalhes os estados puros de sistemas compostos bi-partidos. No Capítulo 2, discutimos as operações locais que diferentes observadores espacialmente separados podem realizar em um estado emaranhado compartilhado. No Capítulo 3, apresentamos nosso método das decomposições sub-Schmidt para estados emaranhados tri-partidos com um qubit. Apresentamos vários exemplos e discutimos suas propriedades. Além disso, mostramos como as diferentes decomposições implicam em diferentes classes de estados emaranhados por transformações do tipo SLOCC. Também apresentamos um critério necessário e suficiente para verificarmos se dois estados pertencem à mesma classe SLOCC. No caso de pertencerem, apresentamos ainda as operações do tipo SLOCC que transformam um estado no outro. 


\section{Capítulo 1}

\section{O emaranhamento quântico}

Neste Capítulo, faremos uma breve introdução ao emaranhamento quântico. Atualmente, o assunto emaranhamento quântico já se tornou bastante vasto. Um boa revisão do desenvolvimento atual foi redigida recentemente por Horodecki et al [15]. Aqui, enfatizaremos mais a descrição das decomposições dos estados emaranhados puros de sistemas compostos bi-partidos de dimensão finita. Também discutiremos o emaranhamento multipartido e algumas aplicações à informação quântica.

\subsection{Estados emaranhados puros bi-partidos}

\subsubsection{O que são estados emaranhados?}

"When two systems, ... enter into temporary physical interaction ... and ... separate again, then they can no longer be described ... by endowing each of them with a representative of its own. I would not call that one but rather the characteristic trait of quantum mechanics, the one that enforces its entire departure from classical lines of thought. By the interaction the two representatives have become entangled."

Schrödinger, 1935 [7]

Emaranhamento é um fenômeno quântico bastante comum quando consideramos diversos sistemas quânticos em conjunto. Esse conjunto de sistemas quânticos, em geral, é chamado de sistema composto e cada um dos sistemas que o constitui de subsistema ou parte do sistema composto. Como é bem conhecido, cada estado de um sistema físico é representado por um raio em um espaço de Hilbert. Assim, como o sistema composto e uma parte deste são sistemas físicos distintos, eles serão associados a representantes distintos em espaços de Hilbert distintos. O conceito de emaranhamento está intimamente 
relacionado com a relação entre esses representantes do estado quântico. Para certos estados do sistemas composto, podemos dizer que os "representantes dos subsistemas estão emaranhados" como disse Schrödinger [8]. Hoje em dia, esses estados são chamados de emaranhados ou dizemos que o sistema possui um certo emaranhamento.

Geralmente, um sistema físico dito composto possui uma grande quantidade de observáveis físicos e pode ser dividido em uma grande quantidade de subsistemas. Essa divisão em muitas partes pode dar origem a estados emaranhados bastante complexos, os quais, ainda aguardam por uma boa teoria que possa descrever suas propriedades de maneira satisfatória [15]. Neste capítulo introdutório nos restringiremos a estudar o caso dos estados puros bipartidos, os quais, são bem conhecidos. Como um exemplo, vamos então começar com o sistema composto mais simples que existe: dois sistemas de dois níveis ou qubits, os quais podem ser realizados através dos spins de duas partículas de spin 1/2 ou da polarização de dois fótons.

Para entendermos como a representação do sistema composto de dois qubits se relaciona com a representação de cada um dos qubits, é conveniente lembrarmos como construir a representação do spin de uma partícula. Neste caso, sabemos experimentalmente que o spin pode assumir dois valores. A cada um destes valores podemos associar um estado quântico, os quais denotaremos por $|0\rangle$ e $|1\rangle$. A partir do princípio da superposição, sabemos que qualquer estado da forma

$$
|\psi\rangle=\alpha|0\rangle+\beta|1\rangle
$$

onde $\alpha$ e $\beta$ são coeficientes complexos tais que $|\alpha|^{2}+|\beta|^{2}=1$, é também um estado válido. Dessa forma, os estados $|0\rangle$ e $|1\rangle$ são associados a uma base ortonormal para um espaço linear de dimensão 2 e qualquer estado do spin de uma partícula pode ser representado por um raio neste espaço.

Podemos agora discutir a representação de um sistema composto de dois qubits. Vamos chamar um dos qubits de subsistema $a, S_{a}$, e o outro de subsistema $b, S_{b}$. Diremos também que $S_{a}$ está com a observadora Alice e $S_{b}$ com o observador Bob. Os dois níveis destes qubits podem ser definidos como autoestados de certos observáveis, $Z_{a}$ para o qubit $S_{a}$ e $Z_{b}$ para o qubit $S_{b}$. Podemos escolher os dois autovalores destes observáveis como \pm 1 . Assim definimos:

$$
\begin{aligned}
Z_{a}\left|0_{a}\right\rangle & =-\left|0_{a}\right\rangle & Z_{b}\left|0_{b}\right\rangle & =-\left|0_{b}\right\rangle \\
Z_{a}\left|1_{a}\right\rangle & =+\left|1_{a}\right\rangle & Z_{b}\left|1_{b}\right\rangle & =+\left|1_{b}\right\rangle .
\end{aligned}
$$

Com essas convenções, $Z_{a}$ na base $\left\{\left|0_{a}\right\rangle,\left|1_{a}\right\rangle\right\}$ é a usual matriz de Pauli utilizada para representar o spin do qubit $S_{a}$ na direção $z$. O mesmo vale para $Z_{b}$ na base $\left\{\left|0_{b}\right\rangle,\left|1_{b}\right\rangle\right\}$.

Uma vez que $Z_{a}$ e $Z_{b}$ correspondem a observações que podem ser feitas independentemente em cada um dos subsistemas, isto é, $Z_{a}$ e $Z_{b}$ são observáveis compatíveis, podemos 
identificar quatro estados simultaneamente distinguíveis para o sistema composto de dois qubits, $S_{a b}$, os quais podem ser denotados por

$$
\left|0_{a}\right\rangle \otimes\left|0_{b}\right\rangle, \quad\left|0_{a}\right\rangle \otimes\left|1_{b}\right\rangle, \quad\left|1_{a}\right\rangle \otimes\left|0_{b}\right\rangle \quad \text { e } \quad\left|1_{a}\right\rangle \otimes\left|1_{b}\right\rangle .
$$

Além disso, pelo princípio da superposição, sabemos que qualquer combinação linear desses estados em (1.1) é também um estado válido do sistema composto. Assim, matematicamente, a representação do sistema composto aparece naturalmente como sendo o espaço linear complexo definido pelo produto tensorial dos espaços lineares dos subsistemas.

O próximo passo é entendermos como os estados dos qubits individuais estão relacionados com o estado do sistema composto de dois qubits, ou seja, queremos responder às seguintes perguntas:

1. Dado o estado do sistema composto, quais são os estados correspondentes dos subsistemas?

2. Dados os estados dos subsistemas, qual o estado correspondente do sistema composto?

Na física clássica, sabemos que um sistema físico composto pode ser representado pelas variáveis canônicas dos subsistemas, ou seja, se uma parte, $S_{a}$, é representada pelas variáveis $\left\{q_{a}, p_{a}\right\}$ e a outra, $S_{b}$, pelas variáveis $\left\{q_{b}, p_{b}\right\}$, o sistema composto, $S_{a b}=S_{a}+S_{b}$, é representado pelas variáveis $\left\{q_{a}, q_{b}, p_{a}, p_{b}\right\}$. Reciprocamente, qualquer estado de um sistema composto clássico pode ser descrito pelas variáveis $\left\{q_{a}, q_{b}, p_{a}, p_{b}\right\}$ e, dada, a representação do sistema composto, podemos inferir imediatamente a representação das partes: $S_{a}$ por $\left\{q_{a}, p_{a}\right\}$ e $S_{b}$ por $\left\{q_{b}, p_{b}\right\}$.

Na mecânica quântica, essa facilidade com que respondemos às perguntas 1 e 2 é um caso bastante particular. Quando a parte $S_{a}$ é representada pelo estado $\left|\psi_{a}\right\rangle$ e a parte $S_{b}$ por $\left|\psi_{b}\right\rangle$, o estado do sistema composto $\left|\psi_{a b}\right\rangle$ é representado por

$$
\left|\psi_{a b}\right\rangle=\left|\psi_{a}\right\rangle \otimes\left|\psi_{b}\right\rangle
$$

e vice-versa. Essa situação pode ser entendida como um análogo da situação clássica quanto à representação dos sistemas compostos. No entanto, uma superposição de dois estados do tipo (1.2), com $\left|\psi_{a}^{\prime}\right\rangle$ e $\left|\psi_{b}^{\prime}\right\rangle$ diferentes de $\left|\psi_{a}\right\rangle$ e $\left|\psi_{b}\right\rangle$, resulta em um estado

$$
\left|\psi_{e m a}\right\rangle=\alpha\left|\psi_{a}\right\rangle \otimes\left|\psi_{b}\right\rangle+\beta\left|\psi_{a}^{\prime}\right\rangle \otimes\left|\psi_{b}^{\prime}\right\rangle
$$

para o sistema composto que não pode ser escrito na forma de um simples produto tensorial como $\left|\psi_{a b}\right\rangle$ em (1.2). Assim, no caso do estado $\left|\psi_{e m a}\right\rangle$, não é possível definir um estado puro para os subsistemas $S_{a}$ e $S_{b}$. Estados como $\left|\psi_{e m a}\right\rangle$, que não podem ser representados 
como um simples produto tensorial como em (1.2) são chamados de estados emaranhados. Os estados que podem ser representados na forma (1.2) são chamados de estados produto ou separáveis. Os quatro estados em (1.1) são estados produto. Superpondo esses estados, podemos escrever quatro exemplos de estados emaranhados que serão bastante úteis,

$$
\begin{aligned}
\left|\psi^{-}\right\rangle & =\frac{1}{\sqrt{2}}\left(\left|0_{a}\right\rangle \otimes\left|1_{b}\right\rangle-\left|1_{a}\right\rangle \otimes\left|0_{b}\right\rangle\right) \\
\left|\psi^{+}\right\rangle & =\frac{1}{\sqrt{2}}\left(\left|0_{a}\right\rangle \otimes\left|1_{b}\right\rangle+\left|1_{a}\right\rangle \otimes\left|0_{b}\right\rangle\right) \\
\left|\phi^{+}\right\rangle & =\frac{1}{\sqrt{2}}\left(\left|0_{a}\right\rangle \otimes\left|0_{b}\right\rangle+\left|1_{a}\right\rangle \otimes\left|1_{b}\right\rangle\right) \\
\left|\phi^{-}\right\rangle & =\frac{1}{\sqrt{2}}\left(\left|0_{a}\right\rangle \otimes\left|0_{b}\right\rangle-\left|1_{a}\right\rangle \otimes\left|1_{b}\right\rangle\right) .
\end{aligned}
$$

Esses quatro estados são chamados de base de Bell e aparecem frequentemente na literatura. O estado $\left|\psi^{-}\right\rangle$foi usado por Bohm [19] na sua reformulação do famoso argumento de incompletude da mecânica quântica de Einstein, Podolski e Rosen [11]. Esses estados também podem ser usados como exemplos da violação das desigualdades de Bell [20] pela mecânica quântica. No campo da informação quântica, esses estados têm papel importante no fenômeno do teletransporte de um estado quântico $[2,4,5]$ e em outras tarefas de informação quântica.

Dessa forma, obtemos uma definição bastante matemática de um estado emaranhado. Dado um sistema composto com um conjunto completo de variáveis dinâmicas $\{A, B\}$, podemos definir os espaços lineares complexos, $\mathcal{H}_{a}$ e $\mathcal{H}_{b}$, gerados pelas bases de autovetores de $A$ e $B,\left\{\left|a_{i}\right\rangle\right\}_{i=1,2,3 \ldots}$ e $\left\{\left|b_{i}\right\rangle\right\}_{i=1,2,3 \ldots}$, dos subsistemas $S_{a}$ e $S_{b}$, respectivamente. Podemos então definir o espaço do sistema composto como $\mathcal{H}_{a} \otimes \mathcal{H}_{b}$. Nessas condições, dizemos que um estado $|\psi\rangle$ do sistema composto é emaranhado se não pode ser representado na forma de um produto tensorial de um estado de $S_{a}$ e outro de $S_{b}$,

$$
|\psi\rangle \neq\left|\psi_{a}\right\rangle \otimes\left|\psi_{b}\right\rangle
$$

onde $\left|\psi_{a}\right\rangle \in \mathcal{H}_{a}$ e $\left|\psi_{b}\right\rangle \in \mathcal{H}_{b}$. É importante ressaltarmos também que $A$ e $B$ também podem representar um conjunto completo de variáveis dinâmicas para os respectivos subsistemas ao invés de uma única variável dinâmica.

Essa definição matemática esconde o conceito físico de emaranhamento. Vamos voltar ao nosso exemplo do sistema composto de dois spins. Ao invés do spin de cada uma das partículas, vamos escolher como conjunto completo os observáveis $A_{\psi, \phi}$ e $B_{ \pm}$atuando na 
base de Bell (1.3) da seguinte forma:

$$
\begin{array}{rlll}
A_{\psi, \phi}\left|\phi^{ \pm}\right\rangle & =\left|\phi^{ \pm}\right\rangle & \text {e } & A_{\psi, \phi}\left|\psi^{ \pm}\right\rangle=-\left|\psi^{ \pm}\right\rangle ;
\end{array}
$$

Ou seja, $A_{\psi, \phi}$ nos diz se o estado do sistema composto é do tipo $\psi$ ou $\phi$ e $B_{ \pm}$nos diz se o estado é do tipo + ou - em (1.2). Dessa forma, podemos definir dois sistemas de dois níveis correspondentes a cada um dos observáveis $A_{\psi, \phi}$ e $B_{ \pm}$. Os estados da base de Bell, nesta partição do sistema composto, são todos estados produtos

$$
\begin{aligned}
& \left|\phi^{+}\right\rangle=|\phi\rangle \otimes|+\rangle, \quad\left|\phi^{-}\right\rangle=|\phi\rangle \otimes|-\rangle, \\
& \left|\psi^{+}\right\rangle=|\psi\rangle \otimes|+\rangle, \quad \text { e } \quad\left|\psi^{-}\right\rangle=|\psi\rangle \otimes|-\rangle \text {. }
\end{aligned}
$$

Isso significa que o fato de que um estado do sistema composto está ou não emaranhado depende de como dividimos o sistema composto em subsistemas, isto é, quais variáveis dinâmicas de um conjunto completo escolhemos como pertencentes às partes $S_{a}$ ou $S_{b}$. E, matematicamente, existe uma infinidade de conjuntos completos para o sistema composto. Então qual conjunto devemos preferir para dizermos que um estado é ou não emaranhado? Essa questão é exatamente a parte física da definição de emaranhamento. É usual considerarmos emaranhamento entre o spin de duas partículas porque podemos dar cada uma das partículas para observadores distintos, digamos Alice e Bob, os quais podem separar-se e então realizar medidas do spin de suas partículas. Nesse sentido, se Alice e Bob compartilham um estado da base de Bell tal que Alice está com uma partícula e Bob está com a outra, eles são capazes de usar esse emaranhamento para verificar as desigualdades de Bell, teletransportar um estado quântico entre outras tarefas que não seriam possíveis classicamente. No entanto, para que algum observador possa medir os observáveis $A_{\psi, \phi}$ e $B_{ \pm}$, ele precisa ter as duas partículas consigo ou precisa fazer as partículas interagirem antes da observação. Assim, não é possível separar espacialmente os "subsistemas" definidos pelos observáveis $A_{\psi, \phi}$ e $B_{ \pm}$. Também é difícil entendermos fisicamente os observáveis $A_{\psi, \phi} \mathrm{e}$ $B_{ \pm}$independentemente. É comum utilizararmos uma medida na base de Bell, a qual pode ser entendida com envolvendo uma medida em conjunto dos observáveis $A_{\psi, \phi}$ e $B_{ \pm}$. Um exemplo é o teletransporte [2] e um caso mais complicado é o da computação baseada em medidas [21]. No entanto, não conhecemos nenhum caso em que a medida de apenas $A_{\psi, \phi}$ ou $B_{ \pm}$tem algum significado ou importância.

Dessa forma, devemos escolher os observáveis $A$ e $B$ de forma que estes possam ser medidos por observadores espacialmente separados. É bastante comum que $A$ e $B$ sejam observáveis de partículas distintas como o spin de duas partículas. No entanto, o emaranhamento quântico também vem sendo estudado em sistemas de partículas idêntidas $[22,23]$. Nestes casos, os subsistemas não estão associados a uma partícula bem definida 
e sim ao número de partículas em determinado estado.

\subsubsection{Correlações Quânticas em estados emaranhados: estados relativos}

Uma característica dos estados emaranhados é a presença de correlações entre as observações realizadas por Alice e Bob nos seus respectivos subsistemas. Quando temos um sistema composto clássico e temos o conhecimento completo deste, não existem correlações entre os subsistemas. No entanto, na mecânica quântica, quando o estado sistema composto é puro e emaranhado, os observáveis dos subsistemas apresentam correlações.

Para começarmos a entender essas correlações, vamos supor que a Alice e Bob compartilhem um sistema quântico bi-partido, isto é, Alice possui uma parte do sistema quântico que pode ser descrito por um observável $A$ com possíveis auto-valores $a_{i}$ e, similarmente, Bob possui a outra parte do sistema descrito por um observável $B$ e possíveis valores $b_{i}$. Assim, um estado qualquer $|\psi\rangle$ do sistema composto pode ser representado na base produto $\left\{\left|a_{i}\right\rangle \otimes\left|b_{j}\right\rangle\right\}$

$$
|\psi\rangle=\sum_{i j} c_{i j}\left|a_{i}\right\rangle \otimes\left|b_{j}\right\rangle
$$

onde $\left|a_{i}\right\rangle$ é o autovetor de $A$ com autovalor $a_{i},\left|b_{i}\right\rangle$ é o autovetor de $B$ com auto-valor $b_{i}$ e $c_{i j}$ são os coeficientes complexos da expansão de $|\psi\rangle$ nesta base. Por termos escolhido a base dos autovetores de $A$, podemos reescrever facilmente $|\psi\rangle$ de maneira a explicitarmos as correlações que Alice obtém observando o valor de $A$,

$$
\begin{aligned}
|\psi\rangle & =\sum_{i} \sqrt{p_{i}}\left|a_{i}\right\rangle \otimes\left(\frac{1}{\sqrt{p_{i}}} \sum_{j} c_{i j}\left|b_{j}\right\rangle\right) \\
& =\sum_{i} \sqrt{p_{i}}\left|a_{i}\right\rangle \otimes\left|r_{a_{i}}\right\rangle
\end{aligned}
$$

onde

$$
\left|r_{a_{i}}\right\rangle=\frac{1}{\sqrt{p_{i}}} \sum_{j} c_{i j}\left|b_{j}\right\rangle \quad \text { e } \quad p_{i}=\sum_{j}\left|c_{i j}\right|^{2}
$$

é uma constante de normalização. Assim, se Alice obtém o autovalor $a_{i}$ na sua observação correspondente ao auto-estado $\left|a_{i}\right\rangle$, ela pode inferir que o estado do subsistema do Bob é $\left|r_{a_{i}}\right\rangle$. Os estados $\left\{\left|r_{a_{i}}\right\rangle\right\}$ foram introduzidos primeiramente por Schrödinger [8] e, também, posteriormente na tese de Everett [9], o qual os chamou de estados relativos. É importante notarmos que os estados $\left\{\left|r_{a_{i}}\right\rangle\right\}$ não são necessariamente ortogonais entre si, podendo tanto serem todos iguais, o que correspondente ao caso em que não existem correlações entre os subsistemas $S_{a}$ e $S_{b}$, i. e., $|\psi\rangle$ é um estado produto, quanto ortogonais o que corresponde a um caso particular bastante interessante que será discutido na seção 1.1.4.

Devemos notar também que, como a notação já sugere, cada estado $\left|r_{a_{i}}\right\rangle$ depende 
apenas do estado $\left|a_{i}\right\rangle$, sendo independente dos outros elementos da base $\left\{\left|a_{j \neq i}\right\rangle\right\}$ e do observável $A$. Particularmente, podemos definir $\left|r_{a_{i}}\right\rangle$ de forma independente destes outros elementos como

$$
\left|r_{a_{i}}\right\rangle=\frac{1}{\sqrt{p_{i}}}\left\langle a_{i} \mid \psi\right\rangle_{\mathcal{H}_{S_{a}}},
$$

onde $\left\langle a_{i} \mid \psi\right\rangle_{\mathcal{H}_{s_{a}}}$ representa o produto escalar parcial no subsistema de Alice, o qual resulta em um estado no subsistema de Bob. Matematicamente, podemos entender esse produto escalar parcial como uma aplicação linear definida por $|\psi\rangle$ do espaço dual do subsistema $S_{a}, \mathcal{H}_{S_{a}}^{*}$, em $\mathcal{H}_{S_{b}}$. Essa interpretação será bastante útil no estudo dos sistemas compostos tri-partidos desenvolvido no Capítulo 3. Assim, essa aplicação linear mapeia o resultado da medida obtida por Alice com o respectivo estado do subsistema de Bob.

Outra observação relevante é que essas correlações não são necessariamente recíprocas, isto é, o estado relativo de $\left|r_{a_{i}}\right\rangle$ em geral não é o estado $\left|a_{i}\right\rangle$. Para vermos isso, basta considerarmos o produto escalar parcial no subsistema de Bob

$$
\left\langle r_{a_{l}} \mid \psi\right\rangle_{\mathcal{H}_{s_{b}}}=\left|a_{l}\right\rangle+\sum_{i \neq l} \sqrt{p_{i}}\left|a_{i}\right\rangle\left\langle r_{a_{i}} \mid r_{a_{l}}\right\rangle
$$

Portanto, $\left|a_{l}\right\rangle$ e $\left|r_{a_{l}}\right\rangle$ serão reciprocamente relativos se, e somente se, o estado relativo $\left|r_{a_{l}}\right\rangle$ for ortogonal aos outros estados relativos $\left|r_{a_{i}}\right\rangle$ para $i \neq l$. Como veremos na seção 1.1.4, essa situação corresponde uma escolha particular do estado $\left|a_{l}\right\rangle$, na qual ele é um elemento da chamada base Schmidt.

\subsubsection{O estado dos subsistemas}

Na seção 1.1.1, deixamos em aberto a questão do estado dos subsistemas quando o sistema composto está emaranhado. Nesta seção vamos discutir esta questão. Como vimos naquela seção, os sistemas físicos $S_{b}$ e $S_{a b}$ são sistemas físicos distintos e possuem dois representantes distintos. No entanto, dado um observável $\mathcal{B}$ do subsistema $S_{b}$, ele é também um observável do sistema composto $S_{a b}$. Assim sendo, temos duas representações para um mesmo observável, ou seja, duas maneiras de calcularmos o valor esperado de $\mathcal{B}$. Como o valor esperado é um dado empírico, ele deve ser independente da representação escolhida e, portanto, essas duas maneiras necessariamente devem coincidir. Esse é o nosso ponto de partida para entendermos como descrever o estado de um subsistema de um sistema físico emaranhado.

Vamos escolher um observável qualquer do subsistema do Bob $\mathcal{B}$. Esse observável é representado no subsistema pelo operador $B$. No sistema composto, esse observável deve ser representado pelo operador $I_{a} \otimes B$, pois, para qualquer estado produto $\left|\psi_{a}\right\rangle \otimes\left|\psi_{b}\right\rangle$, o 
valor médio de $\mathcal{B}$ deve ser independente do estado do subsistema de Alice $\left|\psi_{a}\right\rangle$, isto é,

$$
\langle\mathcal{B}\rangle=\left\langle\psi_{a}\left|\otimes\left\langle\psi_{b}\left|I_{a} \otimes B\right| \psi_{a}\right\rangle \otimes\right| \psi_{b}\right\rangle=\left\langle\psi_{b}|B| \psi_{b}\right\rangle .
$$

Utilizando a decomposição em estados relativos (1.4), fica fácil calcularmos valor médio de $\mathcal{B}$ para um estado arbitrário $|\psi\rangle$ do sistema composto,

$$
\begin{aligned}
\langle\mathcal{B}\rangle & =\operatorname{Tr}\left[|\psi\rangle\langle\psi| I_{a} \otimes B\right] \\
& =\sum_{n m}\left\langle a_{n}\right| \otimes\left\langleb _ { m } \left|( \sum _ { i ^ { \prime } } \sqrt { p _ { i ^ { \prime } } } | a _ { i ^ { \prime } } \rangle \otimes | r _ { a _ { i ^ { \prime } } } \rangle ) \left(\sum_{i} \sqrt{p_{i}}\left\langle a_{i}\left|\otimes\left\langle r_{a_{i}}\right|\right) I_{a} \otimes B \mid a_{n}\right\rangle \otimes\left|b_{m}\right\rangle\right.\right.\right.
\end{aligned}
$$

A soma em $n$, das componentes $\left|a_{n}\right\rangle$, é chamada de traço parcial sobre o subsistema e resulta no produto $\delta_{n, i} \delta_{n, i^{\prime}}$. O valor médio de $\mathcal{B}$ fica

$$
\langle\mathcal{B}\rangle=\sum_{m}\left\langle b_{m}\left|\sum_{i} p_{i}\right| r_{a_{i}}\right\rangle\left\langle r_{a_{i}}|B| b_{m}\right\rangle
$$

que representa um traço no subsistema de Bob. Podemos então definir o operador

$$
\rho_{b}=\sum_{i} p_{i}\left|r_{a_{i}}\right\rangle\left\langle r_{a_{i}}\left|=\operatorname{Tr}_{\mathcal{H}_{a}}\right| \psi\right\rangle\langle\psi|,
$$

onde $\operatorname{Tr}_{\mathcal{H}_{a}}$ denota o traço parcial sobre o espaço do subsistema $S_{a}$, e reescrever o valor médio

$$
\langle\mathcal{B}\rangle=\operatorname{Tr}\left[\rho_{b} B\right]
$$

O operador definido em (1.5) é chamado de operador densidade reduzido e tem a mesma interpretação de uma mistura estatística. Assim, os fatores de normalização $p_{i}$ podem ser interpretados como os pesos da mistura dos estados $\left|r_{a_{i}}\right\rangle$. Como existe uma infinidade de possíveis decomposições em estados relativos, podemos, então, dizer que os estados relativos representam diferentes maneiras de se construir a mistura $\rho_{b}$. Dessa interpretação, concluímos, ainda, que o valor de qualquer dos $p_{i}$ 's deve estar entre o menor e maior autovalor de $\rho_{b}$, pois, caso contrário, poderia haver um estado com probabilidade maior (menor) que a do autoestado com maior (menor) autovalor.

Esse resultado representa uma grande quebra do pensamento clássico, pois embora tenhamos todo o conhecimento possível do sistema composto segundo a mecânica quântica, representado pelo estado puro $|\psi\rangle$, não somos capazes de descrever completamente o estado de um subsistema. Em alguns casos, como nos estados da base de Bell (1.3), a matriz densidade reduzida de um subsistema é proporcional à identidade que, estatisticamente, representa um estado de máxima entropia. Dessa forma, nossa descrição dos subsistemas fica restrita a correlações entre os mesmos, as quais descrevemos na seção 1.1.2. Em 1966, 
Bell [20] mostrou que essas correlações são maiores do que se poderia encontrar em qualquer sistema físico descrito pela física clássica ${ }^{1}$.

\subsubsection{A Representação de Schmidt ou bi-ortogonal}

Nesta seção, vamos mostrar que é sempre possível escolhermos uma determinada base $\left\{\left|\lambda_{i}^{a}\right\rangle\right\}$ para o subsistema de Alice tal que os respectivos estados relativos $\left\{\left|r_{\lambda_{i}^{a}}\right\rangle\right\}$ também sejam ortogonais entre si. Isso nos permitirá escrever o estado emaranhado de uma forma em que as correlações ficam mais evidentes e que, além disso, é extremamente útil.

Uma maneira simples de obtermos esse resultado é calculando a matriz densidade de Alice em uma base $\left|a_{i}\right\rangle$. De (1.4) obtemos

$$
\begin{aligned}
\rho_{a} & =\operatorname{Tr}_{b}[|\psi\rangle\langle\psi|] \\
& =\sum_{j}\left\langleb _ { j } \left|( \sum _ { i } \sqrt { p _ { i } } | a _ { i } \rangle \otimes | r _ { a _ { i } } \rangle ) \left(\sum_{i^{\prime}} \sqrt{p_{i^{\prime}}}\left\langle a_{i^{\prime}}\left|\otimes\left\langle r_{a_{i^{\prime}}}\right|\right) \mid b_{j}\right\rangle\right.\right.\right. \\
& =\sum_{i i^{\prime}} \sqrt{p_{i} p_{i^{\prime}}}\left\langle r_{a_{i^{\prime}}}\left|\left(\sum_{j}\left|b_{j}\right\rangle\left\langle b_{j}\right|\right)\right| r_{a_{i}}\right\rangle\left|a_{i}\right\rangle\left\langle a_{i^{\prime}}\right| \\
& =\sum_{i i^{\prime}} \sqrt{p_{i} p_{i^{\prime}}}\left\langle r_{a_{i^{\prime}}} \mid r_{a_{i}}\right\rangle\left|a_{i}\right\rangle\left\langle a_{i^{\prime}}\right|
\end{aligned}
$$

onde $\left\{\left|b_{i}\right\rangle\right\}$ é uma base para o subsistema do Bob. Agora, podemos supor que o estados $\left|a_{i}\right\rangle$ 's são os autovetores do operador densidade reduzido $\rho_{a},\left|a_{i}\right\rangle=\left|\lambda_{i}^{a}\right\rangle$. Como sabemos que $\rho_{a}$ deve ser diagonal nesta base, é necessário que os estados relativos de $\left|\lambda_{i}^{a}\right\rangle$ sejam tais que

$$
\left\langle r_{\lambda_{i^{\prime}}^{a}} \mid r_{\lambda_{i}^{a}}\right\rangle=\delta_{i j}
$$

em (1.6) para que tenhamos ${ }^{2}$

$$
\rho_{a}=\sum_{i} p_{i}\left|\lambda_{i}^{a}\right\rangle\left\langle\lambda_{i}^{a}\right|
$$

Da equação (1.5) sabemos também que o operador densidade de Bob, $\rho_{b}$, pode ser representado como uma mistura dos estados relativos e, como sabemos que os $\left|r_{\lambda_{i}^{a}}\right\rangle$ 's constituem uma base ortonormal para esse subsistema, temos que $\rho_{b}$ é diagonal nesta base

$$
\rho_{b}=\sum_{i} p_{i}\left|r_{\lambda_{i}^{a}}\right\rangle\left\langle r_{\lambda_{i}^{a}}\left|=\sum_{i} p_{i}\right| \lambda_{i}^{b}\right\rangle\left\langle\lambda_{i}^{b}\right|
$$

\footnotetext{
[11].

${ }^{1}$ Ou qualquer teoria que obedecesse os princípios de localidade e realidade de Einstein, Podolski e Rosen

${ }^{2}$ Em alguns caso, podemos ter alguns $p_{i}$ 's igual a zero, o que corresponde a $\rho_{a}$ ter um núcleo não trivial. Neste caso, podemos escolher arbitrariamente os autovetores deste núcleo tanto para $\rho_{a}$ e $\rho_{b}$ uma vez que probabilidade de encontrarmos algum estado neste subespaço é nula.
} 
onde fizemos $\left|\lambda_{i}^{b}\right\rangle=\left|r_{\lambda_{i}^{a}}\right\rangle$. Voltando ao estado compartilhado $|\psi\rangle$, podemos obter de (1.4) a representação do mesmo nestas bases,

$$
|\psi\rangle=\sum_{i} \sqrt{p_{i}}\left|\lambda_{i}^{a}\right\rangle \otimes\left|\lambda_{i}^{b}\right\rangle
$$

Essa forma simples (1.7) é conhecida como decomposição de Schmidt [6]. É também dita bi-ortogonal devido a ortogonalidade das bases $\left\{\left|\lambda_{i}^{a}\right\rangle\right\}$ e $\left\{\left|\lambda_{i}^{b}\right\rangle\right\}$, as quais denominaremos de bases de Schmidt. Porém, devemos ressaltar aqui que as bases de Schmidt não são necessariamente bases em $S_{a}$ e $S_{b}$. Mas, elas podem ser ditas bases dos suportes locais de $|\psi\rangle$ em $\mathcal{H}_{S_{a}}$ e $\mathcal{H}_{S_{b}}$, isto é, dos subespaços onde o estado dos subsistemas tem alguma probabilidade diferente de zero de ser encontrado. Obviamente sempre podemos completar as bases dos suportes locais para obtermos bases para $\mathcal{H}_{S_{a}}$ e $\mathcal{H}_{S_{b}}$. Dessa forma, podemos observar que, se as dimensões de $\mathcal{H}_{S_{a}}$ e $\mathcal{H}_{S_{b}}$ forem diferentes, segue de (1.7) que os suportes locais de $|\psi\rangle$ nos dois subsistemas têm a mesma dimensão e, portanto, a partir de agora, suporemos sempre que as dimensões de $\mathcal{H}_{S_{a}}, \mathcal{H}_{S_{b}}$ e de seus respectivos suportes locais são sempre iguais.

Uma situação interessante acontece quando Alice resolve medir um observável diagonal na base de Schmidt, $A_{\lambda}$. Nesse caso, se ela obtiver o resultado $a_{\lambda_{l}}$, correspondente ao estado $\left|\lambda_{l}^{a}\right\rangle$, o estado do sistema composto reduz-se ao elemento da decomposição de Schmidt $\left|\lambda_{l}^{a}\right\rangle \otimes\left|\lambda_{l}^{b}\right\rangle$. Assim, o resultado de uma medida realizada por Bob de um observável também compatível com a base de Schmidt, $B_{\lambda}$, resultará sempre em $b_{\lambda_{l}}$, correspondente ao estado $\left|\lambda_{l}^{b}\right\rangle$, isto é, o sistema apresenta uma correlação perfeita entre os observáveis $A_{\lambda}$ e $B_{\lambda}$. Devido a não-ortonormalidade dos estados relativos, essa correlação perfeita entre observáveis existe apenas para observáveis compatíveis com a base de Schmidt.

A unicidade da decomposição (1.7) dependerá da degenerescência dos autovalores de $\rho_{a}$ ou $\rho_{b}$, pois, no caso não degenerado os autovetores $\left|\lambda_{i}^{a}\right\rangle$ e $\left|\lambda_{i}^{b}\right\rangle$ são definidos unicamente e, portanto, também é única a decomposição (1.7). No entanto, se alguns dos autovalores forem degenerados, haverá uma arbitrariedade na escolha de autovetores ortogonais pertencentes aos subespaços invariantes desses autovalores, mas, escolhido um autovetor $\left|\lambda_{l}^{a}\right\rangle$ com autovalor degenerado $\lambda_{l}$ no subsistema de Alice, automaticamente estará definido o autovetor correspondente $\left|\lambda_{l}^{b}\right\rangle$ através do estado $|\psi\rangle$,

$$
\left|\lambda_{l}^{b}\right\rangle=\left\langle\psi \mid \lambda_{l}^{a}\right\rangle
$$

No caso extremo de degenerescência, os operadores densidades $\rho_{a}$ e $\rho_{b}$ são proporcionais ao operador identidade, assim, qualquer base ortonormal em um dos subsistemas é uma base de Schmidt e cada um de seus elementos está associado, através do estado $|\psi\rangle$, com um elemento de uma base de Schmidt no outro subsistema. Além disso, qualquer observável de 
Alice estará perfeitamente correlacionado com um observável correspondente do subsistema de Bob.

Outro caso bastante particular e importante é quando um dos autovalores $\rho_{a}$ e $\rho_{b}$ é igual a $1, \lambda_{l}=1 \mathrm{e}$, portanto, todos os outros autovalores são nulos, $\lambda_{i \neq l}=0$. Nesse caso, o sistema composto $S_{a b}$ reduz-se a um estado produto $|\psi\rangle=\left|\lambda_{l}^{a}\right\rangle \otimes\left|\lambda_{l}^{b}\right\rangle$ e não existem correlações entre os subsistemas, os quais são puros e podem ser representados pelos respectivos vetores de estado $\left|\lambda_{l}^{a}\right\rangle$ e $\left|\lambda_{l^{\prime}}^{b}\right\rangle$. Dessa forma, o fato de o operador densidade reduzido ter apenas um ou vários autovalores não nulo constitui um critério simples para sabermos se um estado $|\psi\rangle$ de um sistema composto está ou não emaranhado.

\subsubsection{Quantificação do emaranhamento}

Nós vimos na seção anterior 1.1.4 que, quando o estado do subsistema é o de entropia máxima, existe uma correlação perfeita entre qualquer observável de um subsistema com um do outro. Além disso, no caso do sistema composto encontrar-se em um estado produto, o estado dos subsistemas é puro e não existem correlações entre os observáveis dos subsistemas. Intuitivamente, esta interpretação sugere que a, medida em que a entropia do estado dos subsistemas aumenta, aumentam também as correlações quânticas entre os subsistemas. Dessa forma, uma maneira de quantificarmos essas correlações ou o emaranhamento de um estado $|\psi\rangle$ do sistema composto seria através da entropia de von Neumann de um dos subsistemas,

$$
E_{S}(|\psi\rangle)=-\operatorname{Tr}\left[\rho_{r} \log \rho_{r}\right]=-\sum_{i=1}^{N} p_{i} \log p_{i} .
$$

Essa não é a única maneira de quantificarmos o emaranhamento de um estado puro bi-partido. Porém, $E_{S}$ é uma das medidas mais utilizadas. De um ponto de vista mais rigoroso e axiomático [15], para que uma quantidade seja uma medida de emaranhamento, basta que ela não aumente por operações locais com comunicação clássica (LOCC) ${ }^{3}$. Como outras quantidades que podem ser consideradas medidas do emaranhamento podemos citar o posto de Schmidt do estado. Dessa forma, principalmente no caso multi-partido, é possível definirmos uma grande quantidade de medidas. Entretanto, é desejável também que uma medida do emaranhamento tenha uma interpretação física e é por isso que a entropia de von Neumann apresenta-se como uma boa medida. Isso deve-se em grande parte aos protocolos de destilação e diluição do emaranhamento introduzidos por Bennett et al [14].

Embora seja um tópico de bastante importância, nesta tese, não discutiremos muito as medidas do emaranhamento. Usaremos $E_{S}$ como uma referência para compararmos

\footnotetext{
${ }^{3}$ Devemos considerar que essa quantidade não aumenta em média para o caso de operações locais estocásticas com comunicação clássica (SLOCC). No Capítulo 2, discutiremos melhor essas operações.
} 
estados emaranhados de sistemas bi-partidos no Capítulo 2. Discutiremos um pouco mais sobre medidas do emaranhamento para sistemas multi-partidos na seção 1.2.

\subsection{Estados emaranhados multi-partidos}

Nos últimos anos, estados emaranhados multi-partidos vêm sendo produzidos em laboratório envolvendo cada vez mais subsistemas. Cabe destacar os recentes experimentos demonstrando o emaranhamento de oito íons armadilhados realizados por Häffner et al [24] e de seis fótons realizados por Lu et al [25]. Além de representarem um grande avanço da técnica experimental, esses experimentos representam importantes demonstrações dos aspectos menos clássicos da física quântica. Apesar disso, o entendimento teórico do emaranhamento multi-partido ainda deixa muito a desejar. Atualmente, somente estados com poucas partes e baixa dimensionalidade são bem compreendidos [15]. Nesta seção, discutiremos um pouco estas dificuldades em descrevermos as propriedades dos estados emaranhados multi-partidos. Particularmente, focaremos um pouco a discussão na questão da representação do estado quântico emaranhado, pois esta questão está relacionada com as decomposições sub-Schmidt que desenvolveremos no Capítulo 3.

A definição de estado emaranhado puro pode ser facilmente estendida para o caso em que o sistema composto é subdividido em diversos subsistemas. Vamos considerar $N$ observadores $O_{i}, i=1,2, \ldots, N$, e cada um deles possui um subsistema $S_{i}$. Assim sendo, um estado $|\psi\rangle$ do sistema composto destes $N$ subsistemas $S_{i}$ é dito emaranhado se não puder ser escrito na forma de um produto tensorial de $N$ estados dos subsistemas,

$$
|\psi\rangle \neq\left|\psi_{1}\right\rangle \otimes\left|\psi_{2}\right\rangle \otimes \ldots \otimes\left|\psi_{N}\right\rangle
$$

onde $\left|\psi_{i}\right\rangle$ é um estado do subsistema $S_{i}$. O estado de cada subsistema $S_{i}$ pode ser definido de maneira análoga à seção 1.1.3,

$$
\rho_{i}=\operatorname{Tr}_{j \neq i}[|\psi\rangle\langle\psi|],
$$

onde $\operatorname{Tr}_{j \neq i}$ denota o traço parcial sobre os outros subsistemas $S_{j}$ diferentes de $S_{i}$. Dessa forma, o estado $|\psi\rangle$ será emaranhado se, e somente se, pelo menos um dos postos dos operadores densidade reduzidos $\rho_{i}$ for maior que 1 .

Embora tenhamos generalizado facilmente a definição de emaranhamento e o critério de separabilidade para estados puros de sistemas compostos multi-partidos, as facilidades terminam neste ponto. Apesar da grande atenção que a comunidade científica tem dispensado ao emaranhamento quântico [15], pouco de fato é conhecido sobre o emaranhamento multipartido. Essa dificuldade começa já na descrição dos estados emaranhados multi-partidos, pois é difícil representá-los de uma maneira simples. No caso de sistemas compostos bi- 
partidos, nós temos a decomposição de Schmidt que torna simples a representação e o estudo dos estados puros nesses sistemas. No entanto, já para o caso tri-partido, não é possível encontrar uma decomposição similar à de Schmidt para todos os estados. De fato, veremos que um estado emaranhado de um sistema $N$-partido com uma decomposição do tipo da de Schmidt torna-se um caso cada vez mais particular com o aumento do número de partes $N$.

Para entendermos isso melhor, basta fazermos um raciocínio de contagem de parâmetros. Considerando um sistema composto de $N$ qubits $^{4}$, um estado arbitrário $|\psi\rangle$ deste sistema pode ser expandido em uma base qualquer

$$
|\psi\rangle=\sum_{b} c_{b}|b\rangle
$$

onde $b$ são as seqüência binárias de $N$ bits. Existem $2^{N}$ seqüências e $2^{N}$ coeficientes complexos $c_{b}$ na expansão de $|\psi\rangle$. Portanto, precisamos de $\left(2^{N+1}-2\right)$ parâmetros reais para especificar um estado arbitrário em um sistema composto de $N$ qubits.

Vamos escolher agora um estado $\left|\psi_{S}\right\rangle$ deste sistema para o qual existe um decomposição tipo Schmidt, isto é, $\left|\psi_{S}\right\rangle$ pode ser decomposto como

$$
\left|\psi_{S}\right\rangle=\sum_{i=1}^{2} \lambda_{i}\left|s_{i}^{(1)}\right\rangle \otimes\left|s_{i}^{(2)}\right\rangle \otimes \ldots \otimes\left|s_{i}^{(N)}\right\rangle,
$$

onde $\left\{\left|s_{i}^{(j)}\right\rangle\right\}$ é uma base no subsistema $j$ e $\lambda_{i}$ são os coeficientes da expansão de $\left|\psi_{S}\right\rangle$. Vamos agora contar o número de parâmetros necessários para especificarmos $\left|\psi_{S}\right\rangle$. Precisamos de dois $\lambda_{i}$ 's complexos, e dois parâmetros reais para cada um dos $2 N$ elementos das bases de Schmidt dos subsistemas. Considerando ainda que $\left|s_{2}^{(i)}\right\rangle$ é definido como ortogonal a $\left|s_{1}^{(i)}\right\rangle$, o número de parâmetros reais necessários para especificarmos um estado do tipo $\left|\psi_{S}\right\rangle$ é $2 N+2$ parâmetros.

Dessa forma, temos que existe uma diferença que aumenta exponencialmente com $N$ entre o número de parâmetros necessários para especificarmos um estado arbitrário e um estado particular que pode ser decomposto numa decomposição tipo Schmidt $N$-partida como em (1.8).

Uma alternativa para decompormos um estado arbitrário é utilizarmos sucessivas decomposições de Schmidt. De fato, esta alternativa foi desenvolvida por Partovi [16], porém ela nos leva sempre a uma decomposição de qualquer estado $|\psi\rangle$ em um número de estados produto da ordem de $2^{N}$, independentemente de $|\psi\rangle$ ter ou não uma decomposição mais simples. Pelo nosso argumento de contagem de parâmetros, podemos ver que a maioria dos estados de sistemas $N$-partidos realmente necessita de uma decomposição em algo da

\footnotetext{
${ }^{4}$ Este argumento pode ser generalizado facilmente para subsistemas de dimensão arbitrária.
} 
ordem de $2^{N}$ estados produto ${ }^{5}$. Entretanto, seria interessante sabermos quando temos um estado que pode ser decomposto em uma forma mais simples.

Uma alternativa para isso é procurarmos encontrar a decomposição de $|\psi\rangle$ com menor número de estados produto possíveis. Chamaremos estas decomposições de decomposiçôes minimas e o número de estados produto nesta decomposição de posto de Schmidt do estado. Entretanto, não existe um método para encontrar tais decomposições ou determinar o posto de Schmidt de um estado.

Dür et al [17] demonstraram que o número de termos de um estado na sua decomposição mínima não pode aumentar por operações locais. Neste sentido, o número de termos na decomposição mínima pode ser entendido como uma medida do emaranhamento que reduz-se ao posto de Schmidt no caso bi-partido. Com isso, eles também foram capazes de classificar os estados de sistemas compostos de três qubits em duas classes distintas: as classes Greenberger-Horne-Zeilinger ${ }^{6}$ (GHZ) e W. Os estados da classe GHZ caracterizamse por poderem ser decompostos em dois estados produto. O exemplo padrão desta classe é o estado

$$
|G H Z\rangle=\frac{1}{\sqrt{2}}(|000\rangle+|111\rangle) .
$$

Já a classe W caracteriza-se por necessitar de ao menos três estados produto na sua decomposição mínima. O exemplo característico desta classe é o estado

$$
|W\rangle=\frac{1}{\sqrt{3}}(|001\rangle+|010\rangle+|100\rangle) .
$$

Dür et al [17] mostraram também que qualquer estado puro de um sistema de três qubits pertence a uma destas classes e pode ser transformado por operações locais estocásticas e comunicação clássica (SLOCC) em um destes estados. Nós discutiremos as operações do tipos SLOCC no Capítulo 2 e reproduziremos o resultado de Dür et al [17] utilizando nossos métodos no Capítulo 3.

Além disso, podemos facilmente imaginar estados similares aos estados $|G H Z\rangle$ e $|W\rangle$ para sistemas compostos de vários qubits. Definimos os estados $N$-partidos do tipo GHZ como

$$
|G H Z\rangle_{N}=\frac{1}{\sqrt{2}}\left(\bigotimes_{i=1}^{N}|0\rangle+\bigotimes_{i=1}^{N}|1\rangle\right)
$$

e do tipo W como

$$
\left|W_{N}\right\rangle=\frac{1}{\sqrt{N}}(|0 \ldots 001\rangle+|0 \ldots 010\rangle+|0 \ldots 0100\rangle+\ldots+|10 \ldots 00\rangle) .
$$

\footnotetext{
${ }^{5}$ Nielsen e Chuang [4] discutem em mais detalhes esta questão do número de parâmetros e relacionam isso com o fato de que produzir um estado emaranhado arbitrário a partir de um estado produto é uma tarefa de complexidade exponencial.

${ }^{6}$ Esse nome vem de um trabalho de Greenberger, Horne e Zeilinger [26, 27], no qual eles utilizam um estado deste tipo com quatro qubits para obter uma generalização do Teorema de Bell [20].
} 
Além dos estados $\left|G H Z_{N}\right\rangle$ e $\left|W_{N}\right\rangle$, outros estados multi-partidos que merecem destaque são estados cluster definidos por Raussendorf e Briegel [28, 29], os quais são importantes para a computação quântica baseada em medidas.

No entanto, esses estados não são os únicos para sistemas compostos de vários qubits. Para quatro qubits, Verstraete et al [30] mostraram que existem pelo menos nove famílias de estados distintas e não equivalentes por operações locais. Para sistemas compostos de mais qubits, o problema permanece em aberto. Miyake e Verstaetre [31] demonstrarm uma classificação dos estados tri-partidos para sistemas compostos de 2 qubits e um sistema de $n$ níveis. No Capítulo 3, mostraremos um método construtivo para encontrarmos famílias de estados emaranhados de sistemas compostos de dois sistemas de $n$ níveis e um qubit. Entretanto, não seremos capazes de obter uma classificação completa, pois o método que será apresentado falha para alguns estados.

É importante ressaltarmos que recentemente estados multi-partidos de até oito qubits já foram produzidos em laboratório. Leibfreid et al [32] demonstraram a existência de um estado tipo GHZ com seis íons aprisionados e Lu et al [25] com seis fótons. Um estado tipo W com notáveis oito íons também foi demonstrado por Häffner et al [24]. Estados cluster também já foram produzidos com quatro fótons por Walther et al [33] e com seis fótons por Lu et al [25]. Dessa forma, é desejável estendermos o nosso entendimento teórico atual dos estados multi-partidos caracterizando os demais estados para que estes possam ser observados. Além disso, tal entendimento promete ser bastante útil para o desenvolvimento da informação quântica.

\subsection{Informação quântica e emaranhamento}

Em computação clássica, quando queremos avaliar a eficiência de um algoritmo ou a dificuldade de um problema computacional, costumamos avaliar a quantidade de bits de memória necessária para armazenar os dados dos estágios intermediários da computação, assim como a quantidade de operações lógicas necessárias em todo o processo computacional. Dessa forma, memória e operações lógicas podem ser entendidos como os recursos fundamentais da computação clássica, pois o custo computacional de um dado algoritmo pode ser medido através do número de bits e do número de portas lógicas ${ }^{7}$ necessárias. Dessa forma, é possível compararmos dois algoritmos comparando o número de bits ou de portas lógicas necessárias para a computação de cada um deles.

Na computação quântica, por analogia à computação clássica, poderíamos dizer que os recursos computacionais fundamentais seriam as operações lógicas quânticas e a memória quântica. O custo computacional quântico poderia então ser medido através do número de portas lógicas de um dado sistema universal e em termos do número de qubits. No entanto,

\footnotetext{
${ }^{7} \mathrm{O}$ sistema universal de portas lógicas mais conhecido é o constituído das portas E, OU e NÃO.
} 
existe um entendimento na comunidade científica de que o principal fator que diferencia a computação clássica da computação quântica é o emaranhamento.

Dessa forma, é natural pensarmos no emaranhamento como um recurso computacional quântico e esta idéia é bastante clara no uso do emaranhamento em tarefas de comunicação quântica. Essa idéia pode ser entendida melhor ao analisarmos dois exemplos simples e bem conhecidos do uso do emaranhamento na comunicação quântica: o caso de teletransporte [2] e do código denso [1] discutidos a seguir. Nas próximas seções descreveremos essas tarefas, as quais servirão também para exemplificar um pouco a idéia de operações locais que será discutida no Capítulo 2.

\subsubsection{Código denso}

O código denso consiste no uso de um estado emaranhado para aumentar a capacidade de comunicação clássica entre Alice e Bob. Primeiramente, devemos observar que a simples introdução de um canal quântico de comunicação não aumenta a capacidade de comunicação clássica, pois, em cada qubit, podemos codificar no máximo um bit de informação em estados ortogonais pré-definidos. Essa situação muda se Alice e Bob compartilharem estados emaranhados. De fato, vamos mostrar que esse código permite dobrarmos a capacidade de comunicação entre eles.

Para mostrarmos isso, suponhamos que Alice e Bob compartilhem o estado emaranhado

$$
\left|\phi^{+}\right\rangle=\frac{1}{\sqrt{2}}(|00\rangle+|11\rangle) .
$$

No caso de uma comunicação de Alice para Bob, Alice realiza uma das seguintes quatro operações no seu qubit: $\sigma_{0}^{a}, \sigma_{1}^{a}, \sigma_{2}^{a}$ ou $\sigma_{3}^{a}$, onde $\sigma_{0}^{a}$ é a operação identidade e $\sigma_{1}^{a}, \sigma_{2}^{a}$ e $\sigma_{3}^{a}$ são os usuais operadores de Pauli nas respectivas direções $x, y$ e $z$. Essas operações resultam respectivamente nos estados da base de Bell

$$
\begin{aligned}
\sigma_{0}^{a}\left|\phi^{+}\right\rangle & =\left|\phi^{+}\right\rangle=\frac{1}{\sqrt{2}}(|00\rangle+|11\rangle) \\
\sigma_{1}^{a}\left|\phi^{+}\right\rangle & =\left|\psi^{+}\right\rangle=\frac{1}{\sqrt{2}}(|10\rangle+|01\rangle) \\
\sigma_{2}^{a}\left|\phi^{+}\right\rangle & =\left|\psi^{-}\right\rangle=\frac{\imath}{\sqrt{2}}(|10\rangle-|01\rangle) \\
\sigma_{3}^{a}\left|\phi^{+}\right\rangle & =\left|\phi^{-}\right\rangle=\frac{1}{\sqrt{2}}(|00\rangle-|11\rangle) .
\end{aligned}
$$

Dessa forma, Alice é capaz de transformar o estado inicial $\left|\phi^{+}\right\rangle$em qualquer um dos outros elementos da base de Bell. Como esses quatro estados são todos ortogonais, eles podem armazenar 2 bits de informação clássica. Isso significa que Alice é capaz de codificar 4 bits de informação clássica no estado do sistema composto atuando apenas no seu qubit. Após 
realizar uma das quatro operações, Alice envia seu qubit a Bob. Então Bob realiza uma medida na base de Bell do sistema composto para descobrir qual operação Alice aplicou no seu qubit, isto é, Bob lê a mensagem de 2 bits realizando uma medida na base de Bell.

Não podemos deixar de ressaltar a importância da coerência e do emaranhamento nesse processo. Por exemplo, em um estado com correlações clássicas como

$$
\rho=\frac{1}{2}(|00\rangle\langle 00|+| 11\rangle\langle 11|)
$$

a aplicação de $\sigma_{3}^{a}$ seria impossível de ser observada por Bob. O mesmo vale para o caso de um qubit transmitido não emaranhado, pois, neste caso a aplicação de $\sigma_{3}^{a}$ introduziria apenas uma fase global nos estados da base $\{|0\rangle,|1\rangle\}$. Dessa forma, temos que são exatamente as correlações puramente quânticas que nos permitem aumentar a capacidade do canal de comunicação entre Alice e Bob.

\subsubsection{Teletransporte quântico}

O teletransporte é um fenômeno ainda mais surpreendente e sutil que o código denso. Nesse caso, usamos o emaranhamento e um canal clássico de comunicação para transmitir informação quântica sem o canal quântico. Para mostrarmos que isso é possível, vamos supor novamente que Alice e Bob compartilham o estado emaranhado

$$
\left|\phi^{+}\right\rangle=\frac{1}{\sqrt{2}}(|00\rangle+|11\rangle) .
$$

e que Alice deseja transmitir um terceiro qubit em um estado desconhecido $|\psi\rangle$ para Bob, mas não dispõe de um canal quântico de comunicação. Nesse caso, Alice pode fazer uma medida na base de Bell do sistema constituído do seu qubit compartilhado e do qubit que ela quer transmitir. Lembrando que cada um dos estados na base de Bell pode ser obtido a partir do estado $\left|\phi^{+}\right\rangle$através de rotações em um dos qubits, podemos escrever os quatro operadores de projeção nos elementos da base de Bell como

$$
\frac{1}{\sqrt{2}} \sigma_{i}\left|\phi^{+}\right\rangle\left\langle\phi^{+}\right| \sigma_{i}
$$

Assim, quando Alice realiza a observação, ela obtém um dos seguintes estados $i$ não normalizados

$$
\left|p_{i}\right\rangle=\frac{1}{\sqrt{2}} \sigma_{i}\left|\phi^{+}\right\rangle\left\langle\phi ^ { + } \left|\sigma_{i}(|\psi\rangle|00\rangle+|\psi\rangle|11\rangle) .\right.\right.
$$

Como um operador de Pauli agindo no qubit de Alice de um estado de Bell é equivalente ao mesmo operador de Pauli agindo no qubit de Bob (a menos de um fator de fase irrelevante), 
temos que

$$
\begin{aligned}
\left|p_{i}\right\rangle & =\frac{1}{\sqrt{2}} \sigma_{i}\left|\phi^{+}\right\rangle \frac{1}{\sqrt{2}}\left(\left\langle00|+\langle 11|)\left(|\psi\rangle|0\rangle \sigma_{i}|0\rangle+|\psi\rangle|1\rangle \sigma_{i}|1\rangle\right)\right.\right. \\
& =\frac{1}{2} \sigma_{i}\left|\phi^{+}\right\rangle\left(\langle 0 \mid \psi\rangle \sigma_{i}|0\rangle+\langle 1 \mid \psi\rangle \sigma_{i}|1\rangle\right) \\
& =\frac{1}{2} \sigma_{i}\left|\phi^{+}\right\rangle \sigma_{i}|\psi\rangle
\end{aligned}
$$

Dessa forma, podemos ver que o qubit de Bob resulta no estado $\sigma_{i}|\psi\rangle$, o qual é o estado que Alice queria transferir para Bob multiplicado pelo operador de Pauli $\sigma_{i}$ que depende do resultado $i$ da medida realizada por Alice. Assim, Alice precisa informar Bob qual o resultado de sua medida na base de Bell o que custa 2 bits de comunicação clássica. Sabendo o resultado da medida de Alice, Bob aplica a operação $\sigma_{i}$ correspondente no seu qubit obtendo $|\psi\rangle$.

É importante observarmos que a probabilidade de Alice obter cada um dos estados $\left|p_{i}\right\rangle$ é um $1 / 4$ e que esta medida não traz nenhuma informação sobre qual é o estado $|\psi\rangle$. Além disso, ao final do processo, Alice fica com dois qubits em um estado de Bell e qualquer informação sobre o antigo estado $|\psi\rangle$ esté perdida para Alice, o que está de acordo com o teorema da não-clonagem $[4,5,34,35]$. 


\section{Capítulo 2}

\section{Transformações locais dos estados emaranhados}

\subsection{Transformações unitárias locais}

Como uma introdução e um exemplo, vamos começar com as transformações locais mais simples que Alice e Bob podem realizar em um estado emaranhado. Suponhamos que Alice realize uma operação unitária no seu subsistema dada pelo operador $U_{a}$ e, similarmente, Bob realize uma operação unitária $U_{b}$ no subsistema dele. O resultado desta operação combinada é uma operação que pode ser escrita na forma

$$
U_{a} \otimes U_{b}
$$

no sistema composto $S_{a b}$. É evidente que eles podem realizar essas operações sem interagirem seus subsistemas e estando espacialmente separados. Por essa razão este tipo de operação é chamada de operação local unitária (LU).

Nós já vimos um exemplo de uma transformação deste tipo no capítulo anterior, no protocolo do código denso na seção 1.3.1. Nesse protocolo, Alice aplica uma das quatro operações unitárias locais $\sigma_{i}^{a}$ no seu qubit de acordo com a informação que ela quer transmitir. Dessa forma, Alice é capaz de transformar o estado de Bell $\left|\phi^{+}\right\rangle$em qualquer outro elemento da base de Bell (1.3) atuando apenas localmente. Além disso, ela pode reverter essa transformação aplicando novamente o operador $\sigma_{i}^{a}$ no seu qubit. Dessa forma, todos os estados de Bell são equivalente por transformações LU e, por isso, possuem a mesma quantidade de emaranhamento.

No caso de estados emaranhados bipartidos puros, é fácil entendermos a ação de uma 
operação LU. Utilizando a decomposição de Schmidt, temos

$$
\begin{aligned}
U_{a} \otimes U_{b}|\psi\rangle & =U_{a} \otimes U_{b} \sum_{i} \sqrt{p_{i}}\left|\lambda_{i}^{a}\right\rangle \otimes\left|\lambda_{i}^{b}\right\rangle \\
& =\sum_{i} \sqrt{p_{i}}\left(U_{a}\left|\lambda_{i}^{a}\right\rangle\right) \otimes\left(U_{b}\left|\lambda_{i}^{b}\right\rangle\right) .
\end{aligned}
$$

Ou seja, os coeficientes de Schmidt são invariantes por operações LU. Qualquer operação LU atua apenas "rodando" a base de Schmidt. Além disso, se dois estados, $|\psi\rangle$ e $\left|\psi^{\prime}\right\rangle$, possuem os mesmo coeficientes de Schmidt, eles estão relacionados através da operação LU que mapeia as bases de Schmidt de $|\psi\rangle$ nas bases de Schmidt de $\left|\psi^{\prime}\right\rangle$. Dessa forma, a identificação dos coeficientes de Schmidt é um critério necessário e suficiente para sabermos se dois estados $|\psi\rangle$ e $\left|\psi^{\prime}\right\rangle$ podem ser interconvertidos por uma operação LU. Por essa razão, ainda, todos os aspectos do emaranhamento bipartido de estados puros, incluindo medidas do emaranhamento, devem depender apenas desses coeficientes. Como cada operação LU $U_{a} \otimes U_{b}$ é reversível através da operação LU $U_{a}^{\dagger} \otimes U_{b}^{\dagger}$, elas definem um conjunto de classes de estados emaranhados. Dois estados pertencem a mesma classe se estão relacionados por uma operação LU.

Por fim, as operações LU podem ser facilmente generalizadas para sistema multipartidos. Uma transformação do estado emaranhado $N$-partido é uma operação LU se puder ser escrita na forma

$$
U_{1} \otimes U_{2} \otimes \ldots \otimes U_{N}
$$

onde $U_{k}, k=1,2, \ldots, N$, é um operador unitário na parte $k$.

\subsection{Operações locais estocásticas com comunicação clássica}

Operações LU não são as únicas operações que Alice e Bob podem realizar em seus subsistemas. Eles também podem interagir seus subsistemas com sistemas auxiliares locais e realizar medidas nos subsistemas. Para que as medidas de Alice e Bob sejam úteis, é necessário que eles também possam comunicar classicamente o resultado destas medidas um ao outro. Essas habilidades adicionais permitem a Alice e Bob realizar um conjunto de operações mais complexas que as operações LU. Dentro deste conjunto ainda teremos dois tipos de operações:

1. as com probabilidade de sucesso 1, as quais são denominadas simplesmente operações locais com comunicação clássica (LOCC) e são bastante importantes para estados emaranhados bi-partidos. É interessante notarmos que podemos realizar operações com probabilidade de sucesso 1 utilizando medidas como veremos no exemplo 1.

2. as com probabilidade de sucesso $p, 0<p \leq 1$, as quais são denominadas de operações 
locais estocásticas com comunicação clássica (SLOCC). Essas operações são mais úteis no estudo dos sistemas compostos tri-partidos do que nos bi-partidos, como veremos a seguir, e serão importantes no desenvolvimento do Capítulo 3.

Esses dois tipos de transformações locais foram definidos por Bennett et al [18]. Eles também discutiram diversos outros tipos de transformações. Porém, nos restringiremos a estas duas aqui.

Nas próximas seções, descreveremos um exemplo de uma operação do tipo LOCC e outro do tipo SLOCC. Para isso, primeiro introduziremos o formalismo de operações quânticos na próxima seção. Depois discutiremos o caso geral das operações SLOCC que é o principal objetivo deste Capítulo, pois isso será bastante útil nos resultados do Capítulo 3.

\subsubsection{Operações Quânticas}

Para entendermos quais transformações podem ser realizadas com as operações do tipo LOCC e SLOCC, vamos utilizar o formalismo de operações quânticas [4, 5, 36]. Esse formalismo foi inicialmente desenvolvido por Hellwig e Kraus [37, 38, 36] e existe uma vasta literatura sobre o assunto relacionada com o tratamento de sistemas quânticos abertos. Ele será bastante útil para avaliarmos quais operações Alice e Bob podem realizar em um estado emaranhado fazendo uso de sistemas auxiliares e medidas.

Vamos considerar então que Alice possui um sistema físico de interesse, $S_{\text {int }}$, e um sistema auxiliar, $S_{a u x}$. O sistema de interesse encontra-se inicialmente no estado $\rho_{i}$ e sistema auxiliar no estado $\left|e_{0}\right\rangle$. Nós podemos supor o estado do sistema auxiliar como puro sem perda de generalidade, pois um estado misto pode ser sempre interpretado como um estado emaranhado puro de um sistema auxiliar de maior dimensão. Vamos considerar também que Alice é capaz de fazer esses sistemas físicos interagirem através de qualquer operador unitário e é capaz de realizar qualquer observação no sistema auxiliar. Dessa forma, se Alice fizer esses dois sistemas interagirem por um certo tempo, o efeito dessa interação pode ser descrito no sistema composto por uma operação unitária $U$. O estado do sistema de interesse de Alice depois da interação fica

$$
\rho_{f}=\operatorname{Tr}_{a u x}\left[U \rho_{i} \otimes\left|e_{0}\right\rangle\left\langle e_{0}\right| U^{\dagger}\right],
$$

onde $\operatorname{Tr}_{\text {aux }}$ denota o traço parcial sobre o sistema auxiliar. Definindo uma base para o sistema auxiliar $\left\{\left|e_{k}\right\rangle\right\}, k=0,1,2, \ldots, N$, onde $N$ é a dimensão do sistema auxiliar, tal que 
$\left|e_{0}\right\rangle$ é um dos elementos desta base, temos

$$
\begin{aligned}
\rho_{f} & =\sum_{k}^{N}\left\langle e_{k}\left|U \rho_{i} \otimes\right| e_{0}\right\rangle\left\langle e_{0}\left|U^{\dagger}\right| e_{k}\right\rangle \\
\rho_{f} & =\sum_{k}^{N} E_{k} \rho_{i} E_{k}^{\dagger},
\end{aligned}
$$

onde $E_{k}=\left\langle e_{k}|U| e_{0}\right\rangle$ é um operador no sistema de interesse chamado de operador de transição e tem componentes

$$
\left[E_{k}\right]_{i j}=\left\langle\varphi_{i}\left|\otimes\left\langle e_{k}|U| e_{0}\right\rangle \otimes\right| \varphi_{j}\right\rangle
$$

onde $\left\{\left|\varphi_{i}\right\rangle\right\}$ é uma base para o sistema de interesse. A expressão (2.1) é chamada de representação de soma de operadores. Dessa forma, utilizando a expressão (2.1), seremos capazes de descrever a dinâmica do sistema de interesse sem nos preocuparmos com a dinâmica global do sistema composto descrita pelo operador $U$.

Entretanto, precisamos verificar ainda quais restrições o conjunto de operadores $\left\{E_{k}\right\}$ deve obedecer para que um operador unitário $U$ exista. Uma condição importante que (2.1) deve satisfazer é a preservação do traço do operador densidade $\rho_{f}$. Essa condição nos leva a

$$
\begin{aligned}
\operatorname{Tr} \rho_{f} & =\operatorname{Tr}\left[\sum_{k}^{N} E_{k} \rho_{i} E_{k}^{\dagger}\right] \\
1 & =\operatorname{Tr}\left[\sum_{k}^{N} E_{k}^{\dagger} E_{k} \rho_{i}\right]
\end{aligned}
$$

Como essa expressão deve ser válida para qualquer $\rho_{i}$, temos que os operadores $E_{k}$ devem satisfazer a condição

$$
\sum_{k}^{N} E_{k}^{\dagger} E_{k}=I .
$$

A dedução desta condição (2.2) mostra que ela é necessária. Para mostrarmos que ela também é uma condição suficiente, vamos mostrar que é sempre possível, dado $N$ operadores $E_{k}$ satisfazendo (2.2), construir um operador unitário $U$ que implementa a operação quântica utilizando um sistema auxiliar $N$-dimensional. Sejam $\left\{\left|\varphi_{j}\right\rangle\right\}$ uma base para o sistema de interesse e $\left\{\left|e_{k}\right\rangle\right\}$ uma base para o sistema auxiliar, então definimos $U$ atuando nas componentes $\left\{\left|\varphi_{j}\right\rangle \otimes\left|e_{0}\right\rangle\right\}$ da base produto do sistema composto como

$$
U\left|\varphi_{j}\right\rangle \otimes\left|e_{0}\right\rangle=\sum_{k}^{N} E_{k}\left|\varphi_{j}\right\rangle \otimes\left|e_{k}\right\rangle .
$$


Podemos verificar facilmente que a ação de $U$ realiza a operação descrita pela soma de operadores (2.1). Utilizando a base $\left\{\left|\varphi_{j}\right\rangle\right\}$, podemos escrever $\rho_{i}$ como

$$
\rho_{i}=\sum_{m n} c_{m n}\left|\varphi_{m}\right\rangle\left\langle\varphi_{n}\right|
$$

Assim, considerando o sistema auxiliar inicialmente no estado $\left|e_{0}\right\rangle$, a ação de $U$ no sistema de interesse fica

$$
\begin{aligned}
\operatorname{Tr}_{\text {aux }}\left[U \rho \otimes\left|e_{0}\right\rangle\left\langle e_{0}\right| U^{\dagger}\right] & =\operatorname{Tr}_{\text {aux }}\left[\sum_{m n} c_{m n} U\left|\varphi_{m}\right\rangle \otimes\left|e_{0}\right\rangle\left\langle\varphi_{n}\right| \otimes\left\langle e_{0}\right| U^{\dagger}\right] \\
& =\operatorname{Tr}_{\text {aux }}\left[\sum_{m n} c_{m n}\left(\sum_{k}^{N} E_{k}\left|\varphi_{m}\right\rangle \otimes\left|e_{k}\right\rangle\right)\left(\sum_{k^{\prime}}^{N}\left(\left\langle\varphi_{n}\right| E_{k^{\prime}}^{\dagger}\right) \otimes\left\langle e_{k^{\prime}}\right|\right)\right] \\
& =\sum_{k}^{N} E_{k}\left(\sum_{m n} c_{m n}\left|\varphi_{m}\right\rangle\left\langle\varphi_{n}\right|\right) E_{k}^{\dagger} \\
& =\sum_{k}^{N} E_{k} \rho_{i} E_{k}^{\dagger} .
\end{aligned}
$$

Para mostrarmos que $U$ é unitário, verificaremos que a ação de $U$ neste subespaço preserva o produto escalar,

$$
\begin{aligned}
\left\langle\varphi_{j}\left|\otimes\left\langle e_{0}\left|U^{\dagger} U\right| \varphi_{i}\right\rangle \otimes\right| e_{0}\right\rangle & =\left(\sum_{k^{\prime}}^{N}\left(\left\langle\varphi_{j}\right| E_{k^{\prime}}^{\dagger}\right) \otimes\left\langle e_{k^{\prime}}\right|\right)\left(\sum_{k}^{N} E_{k}\left|\varphi_{i}\right\rangle \otimes\left|e_{k}\right\rangle\right) \\
& =\sum_{k}\left\langle\varphi_{j}\left|E_{k}^{\dagger} E_{k}\right| \varphi_{i}\right\rangle \\
& =\left\langle\varphi_{j} \mid \varphi_{i}\right\rangle \\
& =\delta_{i j} .
\end{aligned}
$$

Assim, podemos estender a ação de $U$ a todos os elementos da base $\left\{\left|\varphi_{i}\right\rangle \otimes\left|e_{k}\right\rangle\right\}$ tal que este é de fato unitário atuando em todo o espaço do sistema composto. Dessa forma, temos que a condição (2.2) é necessária e suficiente para que Alice possa utilizar um sistema auxiliar para implementar uma dinâmica no sistema de interesse descrita pela soma de operadores (2.1).

A expressão (2.1) também nos diz o que acontece quando Alice faz uma medida na base $\left\{\left|e_{k}\right\rangle\right\}$ do sistema auxiliar. Caso Alice obtenha o elemento $\left|e_{m}\right\rangle$ desta base, a expressão da soma de operadores (2.1) reduz-se ao elemento

$$
E_{m} \rho_{i} E_{m}^{\dagger}
$$


A probabilidade de Alice obter esse elemento é dado por

$$
p_{m}=\operatorname{Tr}\left[E_{m} \rho_{i} E_{m}^{\dagger}\right]
$$

Assim, o estado final do sistema de interesse de Alice normalizado fica

$$
\rho_{f}=\frac{E_{m} \rho_{i} E_{m}^{\dagger}}{\operatorname{Tr}\left[E_{m} \rho_{i} E_{m}^{\dagger}\right]} .
$$

É importante ressaltarmos que a representação de soma de operadores não é única. Particularmente, os operadores $E_{k}$ em (2.1) resultam de termos escolhido a base $\left\{\left|e_{k}\right\rangle\right\}$ para fazermos a dedução. A escolha de outra base resultaria em outros operadores em (2.1), que evidentemente resultaria na mesma relação entre $\rho_{i}$ e $\rho_{f}$, os quais seriam combinações lineares dos operadores $E_{k}$. Para maiores detalhes recomendamos as referências $[4,5]$.

\subsubsection{Exemplos com dois qubits}

Para ilustrarmos como o formalismo de operações quânticas é útil para entendermos as operações locais que Alice e Bob podem realizar em um estado emaranhado, vamos apresentar dois exemplos simples. O primeiro retiramos do livro de Nielsen e Chuang [4]. O segundo exemplo é, na verdade, o da transformação inversa do primeiro exemplo.

\section{Exemplo 1}

Suponhamos que Alice e Bob compartilham o estado emaranhado

$$
\left|\phi^{+}\right\rangle=\frac{1}{\sqrt{2}}(|00\rangle+|11\rangle)
$$

e desejam transformá-lo no estado

$$
\left|\phi_{1}\right\rangle=\cos \theta|00\rangle+\operatorname{sen} \theta|11\rangle,
$$

onde $0 \leq \theta \leq \pi / 4$.

É fácil vermos que eles sempre podem realizar essa transformação com probabilidade 1. Basta Alice preparar o estado $\left|\phi_{1}\right\rangle$ utilizando dois qubits auxiliares e teletransportar um dos qubits para Bob. No entanto, para ilustrarmos o uso dos operadores quânticos, que nos permitirão obter resultados mais gerais nas próximas seções, vamos dar continuidade a um segundo protocolo fazendo uso desses operadores. Nesse segundo protocolo, Alice 
utiliza um sistema auxiliar para aplicar os seguintes operadores quânticos no seu qubit

$$
M_{1}=\left(\begin{array}{cc}
\cos \theta & 0 \\
0 & \operatorname{sen} \theta
\end{array}\right) \quad \text { e } \quad M_{2}=\left(\begin{array}{cc}
\operatorname{sen} \theta & 0 \\
0 & \cos \theta
\end{array}\right)
$$

É fácil verificarmos que $M_{1}$ e $M_{2}$ satisfazem (2.2). A operação quântica no sistema composto de Alice e Bob resulta

$$
\rho_{f}=M_{1} \otimes I_{b}\left|\phi^{+}\right\rangle\left\langle\phi^{+}\left|M_{1}^{\dagger} \otimes I_{b}+M_{2} \otimes I_{b}\right| \phi^{+}\right\rangle\left\langle\phi^{+}\right| M_{2}^{\dagger} \otimes I_{b},
$$

onde $I_{b}$ é o operador identidade do qubit do Bob. Dessa forma, Alice pode realizar uma medida no sistema auxiliar para saber quais das operações, $M_{1}$ e $M_{2}$, ocorreu. Se Alice obtiver que a operação realizada foi $M_{1}$, temos

$$
M_{1}\left|\phi^{+}\right\rangle=\frac{1}{\sqrt{2}}(\cos \theta|00\rangle+\operatorname{sen} \theta|11\rangle)=\frac{1}{\sqrt{2}}\left|\phi_{1}\right\rangle .
$$

Então ela comunica a Bob que o resultado foi $M_{1}$ e que o estado foi transformado corretamente. É importante observarmos que a probabilidade disso acontecer é 1/2. Caso Alice obtenha $M_{2}$, o estado resultante é

$$
M_{2}\left|\phi^{+}\right\rangle=\frac{1}{\sqrt{2}}(\operatorname{sen} \theta|00\rangle+\cos \theta|11\rangle)=\frac{1}{\sqrt{2}}\left|\phi_{2}\right\rangle
$$

e a primeira impressão é que Alice e Bob falharam em obter o estado $\left|\phi_{1}\right\rangle$. No entanto, esse exemplo é interessante porque eles podem corrigir essa falha, pois $\left|\phi_{1}\right\rangle$ e $\left|\phi_{2}\right\rangle$ possuem os mesmos coeficientes de Schmidt e portanto podem ser relacionados por uma operação LU. Assim, caso Alice obtenha $M_{2}$ ela pode aplicar a operação $\sigma_{x}$ no seu qubit e dizer para Bob aplicar também a operação $\sigma_{x}$ no qubit dele. Dessa forma, eles obtêm o estado $\left|\phi_{1}\right\rangle$ de $\left|\phi_{2}\right\rangle$ e a conversão de $\left|\phi^{+}\right\rangle$para $\left|\phi_{1}\right\rangle$ pode ser feita com probabilidade de sucesso 1.

Esse exemplo mostra que é possível obtermos alguns estados emaranhados a partir de outros mesmo que eles não estejam relacionados por uma operação LU.

\section{Exemplo 2}

Uma questão natural agora é: podemos converter $\left|\phi_{1}\right\rangle$ novamente em $\left|\phi^{+}\right\rangle$? Vamos então tentar encontrar os operadores que transformariam $\left|\phi_{1}\right\rangle$ em $\left|\phi^{+}\right\rangle$. Nesse caso, podemos escrever o operador $M_{1}^{-1}$

$$
M_{1}^{-1}=\sqrt{\frac{p_{1}}{2}}\left(\begin{array}{cc}
\frac{1}{\cos \theta} & 0 \\
0 & \frac{1}{\operatorname{sen} \theta}
\end{array}\right)
$$


onde $p_{1}$ é uma normalização que introduzimos para que possamos satisfazer a eq. (2.2). Com um cálculo simples, temos

$$
M_{1}^{-1}\left|\phi_{1}\right\rangle=\sqrt{p_{1}}\left|\phi^{+}\right\rangle
$$

de onde podemos interpretar $p_{1}$ como a probabilidade de obtermos a operação $M_{1}^{-1}$. Agora temos um operador que faz a operação desejada por Alice e Bob, mas precisamos da operação complementar, a qual pode ser obtida da condição (2.2). Temos

$$
M_{1}^{-1}\left(M_{1}^{-1}\right)^{\dagger}+M_{2}^{-1}\left(M_{2}^{-1}\right)^{\dagger}=I
$$

que implica em

$$
M_{2}^{-1}\left(M_{2}^{-1}\right)^{\dagger}=\left(\begin{array}{cc}
1-\frac{p_{1}}{2 \cos ^{2} \theta} & 0 \\
0 & 1-\frac{p_{1}}{2 \operatorname{sen}^{2} \theta}
\end{array}\right) .
$$

Como $0 \leq \theta \leq \pi / 4$, que implica em $\operatorname{sen} \theta \leq \cos \theta$, e $M_{2}^{-1}\left(M_{2}^{-1}\right)^{\dagger}$ deve ser um operador positivo temos que $p_{1} \leq 2 \operatorname{sen}^{2} \theta$. Dessa forma, temos que a probabilidade máxima de sucesso é $p_{1}=2 \operatorname{sen}^{2} \theta$. No caso de escolhermos $p_{1}$ máximo, temos

$$
M_{2}^{-1}=\left(\begin{array}{cc}
1-\tan ^{2} \theta & 0 \\
0 & 0
\end{array}\right)
$$

e se Alice obtiver $M_{2}^{-1}$ o estado resultante é $|00\rangle$, i. e. , um estado produto. Assim, se Alice obtiver que a operação realizada foi $M_{2}^{-1}$, todo o emaranhamento do estado $\left|\phi_{1}\right\rangle$ é perdido. Podemos ver também que quanto mais próximo $\theta$ estiver de $\pi / 4$, mais próximo $p_{1}$ está de 1 e o estado $\left|\phi_{1}\right\rangle$ possui mais emaranhamento. Isso significa que $p_{1}$ aumenta à medida que $\left|\phi_{1}\right\rangle$ aproxima-se de um estado maximamente emaranhado. Dessa forma, temos que é possível transformar $\left|\phi_{1}\right\rangle$ novamente em $\left|\phi^{+}\right\rangle$por operações locais, mas essa operação tem uma probabilidade de sucesso que depende do emaranhamento de $\left|\phi_{1}\right\rangle$.

\section{Discussão sobre os exemplos}

Esses exemplos revelam uma irreversibilidade nas transformações dos estados emaranhados, pois verificamos que o estado $\left|\phi^{+}\right\rangle$pode ser sempre transformado no estado $\left|\phi_{1}\right\rangle$, mas essa operação não pode ser invertida sempre. Essa idéia está de acordo com a fato de que $\left|\phi^{+}\right\rangle$é um estado maximamente emaranhado e possui mais emaranhamento que $\left|\phi_{1}\right\rangle$. Nesse sentido, interpretamos que perdemos certo emaranhamento na transformação de $\left|\phi^{+}\right\rangle$em $\left|\phi_{1}\right\rangle$ e este emaranhamento não pode ser recuperado com operações locais.

Nielsen [13] demonstrou um critério necessário e suficiente para sabermos se podemos converter um estado puro emaranhado bi-partido $|\psi\rangle$ em outro $\left|\psi^{\prime}\right\rangle$. Esse critério confirma que uma condição necessária para transformarmos $|\psi\rangle$ em $\left|\psi^{\prime}\right\rangle$ é que $|\psi\rangle$ tenha mais ema- 
ranhamento que $\left|\psi^{\prime}\right\rangle$. No entanto essa não é uma condição suficiente e existem estados de sistemas compostos bi-partidos com dimensão maior que 2 que não podem ser convertidos um no outro e vice-versa com probabilidade 1 . Nestes casos, podemos dizer que o emaranhamento destes estados não é comparável por LOCC. Outro resultado importante foi deduzido por Bennett et al [18] e afirma que dois estados são interconversíveis por LOCC se, e somente se, estão relacionados por uma operação LU. Este último resultado estende-se também para estados multipartidos.

\subsubsection{Critério de convertibilidade para SLOCC}

Nesta seção, vamos apresentar um critério para sabermos se dois estados emaranhados podem ser convertidos um no outro através de SLOCC. Esse resultado foi estabelecido por Dür et al [17] e nós vamos separá-lo nos Teoremas 2.1 e 2.2. O Teorema 2.1 nos dirá quando um estado puro pode ser obtido de outro estado também puro por SLOCC e o Teorema 2.2 nós dirá quando dois estados são equivalentes por SLOCC, isto é, quando dois estados podem ser convertidos um no outro por SLOCC. Quando dois estados forem equivalentes, teremos que eles podem ser usados para as mesmas tarefas de informação ou comunicação quântica, mas com probabilidades de sucesso distintas. Além disso, será possível mostrarmos que certas propriedades dos estados emaranhados são invariantes por este tipo de operações.

Teorema 2.1: Um estado puro $|\psi\rangle$ de um sistema composto $N$-partido pode ser transformado em um outro estado puro $\left|\psi^{\prime}\right\rangle$ através de uma operação do tipo SLOCC se, e somente se, existirem operadores lineares locais $L_{i}$ tais que

$$
\left|\psi^{\prime}\right\rangle=L_{1} \otimes L_{2} \otimes \ldots \otimes L_{N}|\psi\rangle
$$

onde $L_{i}$ é um operador linear no subsistema $i, i=1,2, \ldots, N$. Os operadores $L_{i}$ são definidos a menos de uma constante complexa multiplicativa e na eq. (2.3) nós desconsideramos a normalização de $|\psi\rangle$ e $\left|\psi^{\prime}\right\rangle$.

Prova: $(\Rightarrow)$ Vamos mostrar primeiro que se a eq. (2.3) é verdade então podemos construir uma operação do tipo SLOCC que transforma $|\psi\rangle$ em $\left|\psi^{\prime}\right\rangle$. Seja $O_{i}$ o observador que possui o subsistema $i$. Assim, vamos começar com as operações do observador $O_{1}$ que realiza a seguinte operação no seu subsistema:

$$
E_{s}=\sqrt{p_{1}} L_{1} \quad \text { e } \quad E_{f}=\sqrt{I_{1}-p_{1} L_{1} L_{1}^{\dagger}}
$$

onde $I_{1}$ é o operador identidade no subsistema 1 e $0<p_{1} \leq 1 / \lambda_{M}$, onde $\lambda_{M}$ é o maior autovalor do operador positivo $L_{1} L_{1}^{\dagger}$. Dessa forma, $O_{1}$ realiza uma medida para verificar quais das operações $E_{s}$ e $E_{f}$ ele obtém. Caso ele obtenha $E_{s}$, o que ocorre com probabilidade 
máxima $p_{1}$, temos que a operação

$$
L_{1} \otimes I_{2} \otimes \ldots \otimes I_{N}
$$

onde $I_{i}$ é o operador identidade no subsistema $i$, é aplicada ao estado $|\psi\rangle$ e ele comunica aos outros observadores que foi bem sucedido na transformação. Caso $O_{1}$ obtenha $E_{f}$ ele comunica a eles que o protocolo falhou.

Os outros observadores procedem de maneira similar a $O_{1}$. Cada observador $O_{i}$ realiza a operação

$$
E_{s}^{(i)}=\sqrt{p_{i}} L_{i} \quad \text { e } \quad E_{f}^{(i)}=\sqrt{I_{i}-p_{i} L_{i} L_{i}^{\dagger}}
$$

no seu respectivo subsistema, $i=2,3, \ldots, N$, e comunica aos outros observadores se a operação foi bem sucedida, que corresponde a obter a operação $E_{s}^{(i)}$, ou se falhou, que corresponde a obter a operação $E_{f}^{(i)}$. O resultado final, no caso em que todos os observadores são bem sucedidos, é a aplicação da operação

$$
L_{1} \otimes L_{2} \otimes \ldots \otimes L_{N}
$$

em $|\psi\rangle$, a qual transforma-o em $\left|\psi^{\prime}\right\rangle$, como queríamos demonstrar. Ainda temos que a probabilidade de sucesso na operação completa é no máximo

$$
p=\prod_{i=1}^{N} p_{i}
$$

Também verificamos que $1 / p$ é o fator de normalização que ignoramos na eq. (2.3).

$(\Leftarrow)$ Vamos provar agora que a validade da eq. (2.3) é também uma condição necessária. Se cada observador $O_{i}$ faz seu subsistema interagir com um sistema auxiliar, o resultado destas operações pode ser expresso na representação de soma de operadores como

$$
\rho_{f}=\sum_{k_{1}, k_{2}, \ldots, k_{N}} E_{k_{1}}^{(1)} \otimes E_{k_{2}}^{(2)} \otimes \ldots \otimes E_{k_{n}}^{(N)}|\psi\rangle\langle\psi| E_{k_{1}}^{(1) \dagger} \otimes E_{k_{2}}^{(2) \dagger} \otimes \ldots \otimes E_{k_{n}}^{(N) \dagger},
$$

onde $E_{k_{i}}^{(i)}$ são operadores lineares no subsistema $i$. Cada um dos observadores $O_{i}$ pode agora realizar medidas nos seus respectivos sistemas auxiliares e verificar quais operadores $E_{k_{i}}^{(i)}$ foram realizados. Assim, se $\left|\psi^{\prime}\right\rangle$ pode ser obtido de $|\psi\rangle$ por operações SLOCC, então, pelo menos uma das possíveis realizações é tal que

$$
\left|\psi^{\prime}\right\rangle=E_{m_{1}}^{(1)} \otimes E_{m_{2}}^{(2)} \otimes \ldots \otimes E_{m_{n}}^{(N)}|\psi\rangle
$$

onde $E_{m_{i}}^{(i)}$ são os operadores lineares realizados no subsistema $i$.

Nós temos agora um critério para saber se um estado $|\psi\rangle$ pode ser convertido em 
um outro estado $\left|\psi^{\prime}\right\rangle$ por SLOCC. Uma questão importante agora é: Dado que $|\psi\rangle$ pode ser transformado em $\left|\psi^{\prime}\right\rangle$ por SLOCC, quando é possível transformarmos $\left|\psi^{\prime}\right\rangle$ novamente em $|\psi\rangle$ por SLOCC? Para respondermos essa pergunta, é importante entendermos o que acontece com a dimensão dos suportes locais de $|\psi\rangle$ nos subsistemas quando realizamos uma operação do tipo SLOCC. Como esse resultado será importante para a demonstração do Teorema 2.2, vamos enuncia-lo como um Lema.

Lema 2.1: Sejam $|\psi\rangle$ e $\left|\psi^{\prime}\right\rangle$ dois estados $N$-partidos tais que $\left|\psi^{\prime}\right\rangle$ pode ser obtido de $|\psi\rangle$ por SLOCC. Sejam também $\rho_{i}$ e $\rho_{i}^{\prime}, i=1,2, \ldots, N$, os operadores densidade reduzidos do subsistema $i$ para os estados $|\psi\rangle$ e $\left|\psi^{\prime}\right\rangle$, respectivamente. Então,

$$
\operatorname{posto} \rho_{i}^{\prime} \leq \operatorname{posto} \rho_{i}
$$

Prova: Se $\left|\psi^{\prime}\right\rangle$ pode ser obtido por SLOCC de $|\psi\rangle$, então, pelo teorema 2.1, temos que existem operadores lineares locais $L_{i}$ 's atuando nos $N$ subsistemas $i$, satisfazendo (2.3). Vamos supor primeiramente que esses operadores são da forma

$$
L_{1} \otimes I_{2} \otimes I_{3} \otimes \ldots I_{N}
$$

onde $I_{i}$ é o operador identidade no subsistema $i$. Então vamos considerar a decomposição de Schmidt de $|\psi\rangle$ entre o subsistema 1 e os outros subsistemas,

$$
|\psi\rangle=\sum_{i}^{n_{1}} \lambda_{i}\left|\lambda_{1}^{(1)}\right\rangle \otimes\left|\lambda_{i}^{(2-N)}\right\rangle
$$

onde $\left\{\left|\lambda_{i}^{(1)}\right\rangle\right\}$ e $\left\{\left|\lambda_{i}^{(2-N)}\right\rangle\right\}$ são as respectivas bases de Schmidt no espaço do subsistema 1 e no espaço dos outros subsistemas e $n_{1}$ é o número de termos da decomposição. Assim, sabemos que

$$
n_{1}=\text { posto } \rho_{1}=\text { posto } \rho_{2-N},
$$

onde $\rho_{2-N}$ é o operador densidade reduzido dos outros subsistemas. Independentemente de qual seja o operador $L_{1}$, a ação de $L_{1}$ no suporte de $\rho_{1}$ pode ser descrita como

$$
L_{1}=\sum_{i}^{n_{1}}\left|\varphi_{i}\right\rangle\left\langle\lambda_{i}^{(1)}\right|,
$$

onde $\left|\varphi_{i}\right\rangle$ são estados arbitrários no espaço do subsistema 1 . Dessa forma, temos que

$$
\rho_{1}^{\prime}=L_{1} \rho_{1} L_{1}^{\dagger}=\lambda_{i}\left|\varphi_{i}\right\rangle\left\langle\varphi_{i}\right|
$$

Assim, se $\left\{\left|\varphi_{i}\right\rangle\right\}$ for um conjunto linearmente independente teremos posto $\rho_{1}^{\prime}=n_{1}$ e, caso contrário, posto $\rho_{1}^{\prime}<n_{1}$. Portanto, posto $\rho_{1}^{\prime} \leq \operatorname{posto} \rho_{1}=n_{1}$. 
Além disso, por causa da decomposição de Schmidt, temos também que

$$
\text { posto } \rho_{2-N}^{\prime} \leq \operatorname{posto} \rho_{2-N},
$$

onde $\rho_{2-N}^{\prime}$ é o operador densidade reduzido de $\left|\psi^{\prime}\right\rangle$ nos subsistemas 2 até $N$. Dessa forma, podemos utilizar o mesmo procedimento para mostrar que uma operação SLOCC em qualquer um dos outros subsistemas também não pode aumentar o posto de $\rho_{1}$.

Além disso, podemos proceder de forma análoga para os subsistemas 2 até $N$. Como uma operação SLOCC pode ser escrita como o produto

$$
\left(L_{1} \otimes I_{2} \otimes I_{3} \otimes \ldots I_{N}\right)\left(I_{1} \otimes L_{2} \otimes I_{3} \otimes \ldots I_{N}\right) \ldots\left(I_{1} \otimes I_{2} \otimes I_{3} \otimes \ldots L_{N}\right)
$$

temos que posto $\rho_{i}^{\prime} \leq \operatorname{posto} \rho_{i}$.

Utilizando o Lema 2.1, podemos ver que, para que dois estados puros $N$-partidos $|\psi\rangle$ e $\left|\psi^{\prime}\right\rangle$ sejam equivalentes, é necessário que a dimensão dos suportes locais $|\psi\rangle$ e $\left|\psi^{\prime}\right\rangle$ em cada um dos subsistemas seja idêntica. Esse fato é uma das motivações para introdução do conceito de dimensionalidade do emaranhamento. Além disso, essa definição simplificará o tratamento dos estados emaranhados na seqüência deste Capítulo e no Capítulo 3.

Definição 2.1: (dimensionalidade do emaranhamento) Vamos dizer que um estado emaranhado puro $N$-partido $|\psi\rangle$ tem dimensionalidade $n_{1}$ por $n_{2}$ por $\ldots$ por $n_{N}$, ou que $|\psi\rangle$ é um estado emaranhado com dimensionalidade $n_{1}$ por $n_{2}$ por ... por $n_{N}$ se seus respectivos suportes locais no espaço dos subsistemas $\mathcal{H}_{1}, \mathcal{H}_{2}, \ldots \mathcal{H}_{N}$ tiverem dimensão $n_{1}, n_{2}, \ldots, n_{n}$, respectivamente. Além disso, vamos denotar a dimensionalidade do emaranhamento por $\left(n_{1}, n_{2}, \ldots, n_{N}\right)$.

(dimensionalidade maior e menor) Também diremos que um estado $|\psi\rangle$ de dimensionalidade $\left(n_{1}, n_{2}, \ldots, n_{N}\right)$ tem dimensionalidade menor (ou maior) que um outro estado $\left|\psi^{\prime}\right\rangle$ de dimensionalidade $\left(n_{1}^{\prime}, n_{2}^{\prime}, \ldots, n_{N}^{\prime}\right)$ se $n_{i} \leq n_{i}^{\prime}$ (ou $n_{i} \geq n_{i}^{\prime}$ ) e pelo menos para algum $i, n_{i}<n_{i}^{\prime}\left(\right.$ ou $\left.n_{i}>n_{i}^{\prime}\right)$.

Esta definição é útil porque, para entendermos o emaranhamento de um estado $N$ partido $|\psi\rangle$, a dimensão dos suportes locais é mais importante do que a dimensão dos subsistemas. De fato, a dimensão do espaço dos subsistemas só é importante para estabelecer um limite superior para a dimensionalidade do estado emaranhado. Sendo assim, consideramos mais simples especificar logo os suportes locais de $|\psi\rangle$ em cada subsistemas e procederemos desta forma no restante desta tese.

Além disso, já podemos introduzir uma simplificação no nosso formalismo de operadores quânticos para operações locais. Quando consideramos o operador $L_{i}$ no Teorema 2.1, ele pode atuar no espaço inteiro do subsistema $i$. No entanto, a ação de $L_{i}$ fora do suporte local de $|\psi\rangle$ no subsistema $i$ é totalmente irrelevante. Então, no que diz respeito ao estado emaranhado $|\psi\rangle$ e as possíveis transformações locais que podem ser realizadas neste estado, 
podemos considerar a dimensão do subsistema $i$ igual a dimensão do suporte local de $|\psi\rangle$ e os operadores $L_{i}$ agindo apenas sobre o suporte local de $|\psi\rangle$ dos respectivos subsistemas. A demonstração do Teorema 2.2 também fica mais simples utilizando esta convenção.

Teorema 2.2: Dois estados puros $N$-partidos $|\psi\rangle$ e $\left|\psi^{\prime}\right\rangle$ de mesma dimensionalidade são equivalentes por SLOCC se, e somente se, existirem operadores lineares inversiveis $L_{i}$ tais que

$$
\left|\psi^{\prime}\right\rangle=L_{1} \otimes L_{2} \otimes \ldots \otimes L_{N}|\psi\rangle,
$$

onde $L_{i}$ é um operador linear inversivel no subsistema $i, i=1,2, \ldots, n$. Os operadores $L_{i}$ novamente são definidos a menos de uma constante complexa multiplicativa e nós desconsideramos a normalização de $|\psi\rangle$ e $\left|\psi^{\prime}\right\rangle$.

Prova: $(\Rightarrow)$ Vamos demonstrar primeiro que, se $|\psi\rangle$ e $\left|\psi^{\prime}\right\rangle$ são equivalentes por SLOCC, então os operadores $L_{i}$ são todos inversíveis. Novamente, começaremos demonstrando o caso particular em que apenas o observador 1 realiza operações no seu subsistema. Mostraremos que, se

$$
\left|\psi^{\prime}\right\rangle=L_{1} \otimes I_{2} \otimes \ldots \otimes I_{n}|\psi\rangle
$$

e $|\psi\rangle$ pode ser obtido novamente de $\left|\psi^{\prime}\right\rangle$ por SLOCC, então

$$
|\psi\rangle=L_{1}^{-1} \otimes I_{2} \otimes \ldots \otimes I_{n}|\psi\rangle,
$$

onde $I_{i}$ é o operador identidade no subsistema $i$. Para tanto, suponhamos que $L_{1}$ não seja inversível. Neste caso, a dimensão suporte local de $\left|\psi^{\prime}\right\rangle$ no subsistema 1 seria igual ao posto de $L_{1}$ que é menor que a dimensão do suporte local de $|\psi\rangle$ no subsistema 1. Portanto, $\left|\psi^{\prime}\right\rangle$ não poderia ser transformado novamente em $|\psi\rangle$ por SLOCC, pois sabemos, pelo Lema 2.1, que a dimensão do suporte local de $\left|\psi^{\prime}\right\rangle$ não pode aumentar. Assim, é necessário que $L_{1}$ seja um operador inversível. Podemos proceder de forma análoga para os subsistemas 2 até $N$ e o produto destas operações é a operação desejada e todos os operadores $L_{i}$ são inversíveis.

$(\Leftarrow)$ Uma vez que existem operadores $L_{i}$ inversíveis relacionando $|\psi\rangle$ e $\left|\psi^{\prime}\right\rangle$ em (2.4) temos, pelo Teorema 2.1, que $|\psi\rangle$ pode ser transformado em $\left|\psi^{\prime}\right\rangle$ e vice-versa por SLOCC.

Uma conseqüência importante do Teorema 2.2 é o fato de que o posto de Schmidt de um estado $N$-partido $|\psi\rangle$ é invariante por operações do tipo SLOCC. Para mostrarmos isso, basta escrevermos $|\psi\rangle$ na sua decomposição mínima

$$
|\psi\rangle=\sum_{i}^{R} c_{i}\left|\varphi_{i}^{(1)}\right\rangle \otimes\left|\varphi_{i}^{(2)}\right\rangle \otimes \ldots \otimes\left|\varphi_{i}^{(N)}\right\rangle,
$$

onde $R$ é número mínimo de estados produto no qual $|\psi\rangle$ pode ser decomposto, $c_{i}$ são 
os coeficientes apropriados da expansão e $\left|\varphi_{i}^{(j)}\right\rangle$ são estados do subsistema $j$. A ação de operadores locais inversíveis em $|\psi\rangle$ fica

$$
\left|\psi^{\prime}\right\rangle=L_{1} \otimes L_{2} \otimes \ldots L_{N}|\psi\rangle=\sum_{i}^{R} c_{i} L_{1}\left|\varphi_{i}^{(1)}\right\rangle \otimes L_{2}\left|\varphi_{i}^{(2)}\right\rangle \otimes \ldots \otimes L_{N}\left|\varphi_{i}^{(N)}\right\rangle .
$$

Dessa forma, $\left|\psi^{\prime}\right\rangle$ também terá uma decomposição mínima com $R$ estados produto.

Além disso, esse resultado motivou Eisert e Briegel [?] a definirem uma medida do emaranhamento multi-partido baseada no posto de Schmidt do estado emaranhado. Esse fato também é uma das motivações para buscarmos as decomposições mínimas dos estados tri-partidos como faremos no Capítulo 3.1.

\subsubsection{Exemplo: Classificação SLOCC para o caso bi-partido}

Como um exemplo da aplicação dos resultados gerais obtidos na seção anterior 2.2.3, desenvolveremos a classificação dos estados puros de sistemas compostos bi-partidos por SLOCC. Essa classificação foi introduzida por Dür et al [17] e é bastante simples. Nós vimos na seção 2.2.3, que o posto de Schmidt de um estado quântico é invariante por SLOCC. No caso dos sistemas bi-partidos, mostraremos que Alice e Bob podem transformar, utilizando operações do tipo SLOCC, um estado com posto de Schmidt $n$ em qualquer outro estado com posto de Schmidt menor ou igual a $n$.

Esse resultado pode ser obtido construindo um operador linear que faz a transformação desejada. Suponhamos que Alice e Bob compartilhem um estado puro bi-partido $|\psi\rangle$ com posto de Schmidt $n$. O estado $|\psi\rangle$ pode ser escrito na sua decomposição de Schmidt

$$
|\psi\rangle=\sum_{i}^{n} \sqrt{\lambda_{i}}\left|a_{i}\right\rangle \otimes\left|b_{i}\right\rangle
$$

onde escolhemos arbitrariamente as bases de Schmidt $\left\{\left|a_{i}\right\rangle\right\}$ e $\left\{\left|b_{i}\right\rangle\right\}$ nos subsistemas de Alice e Bob. Como podemos transformar estas bases em quaisquer outras duas bases através de operações LU, basta construirmos um operador que leve os coeficientes de Schmidt $\sqrt{\lambda_{i}}$ em coeficientes arbitrários $\sqrt{\lambda_{i}^{\prime}}$. Definimos então o operador linear local

$$
A \otimes I_{b}
$$

onde $I_{b}$ é o operador identidade no subsistema de Bob e

$$
A=\sum_{i=1}^{n^{\prime}} \sqrt{\frac{\lambda_{i}^{\prime}}{\lambda_{i}}}\left|a_{i}\right\rangle\left\langle a_{i}\right|
$$


Dessa forma, temos

$$
\left|\psi^{\prime}\right\rangle=A \otimes I_{b}|\psi\rangle=\sum_{i=1}^{n^{\prime}} \sqrt{\lambda_{i}^{\prime}}\left|a_{i}\right\rangle \otimes\left|b_{i}\right\rangle,
$$

onde $n^{\prime}$ é o posto de Schmidt de $\left|\psi^{\prime}\right\rangle$ que pode ser menor ou igual a $n$ dependendo de $A$.

Dessa forma, para estados puros de sistemas bi-partidos, temos uma classificação completa dos estados emaranhados caracterizada pelo posto de Schmidt do estado. Além disso, temos também uma hierarquia destas classes, pois os estados com posto de Schmidt $n$ podem ser transformados em quaisquer estados com posto de Schmidt menor que $n$. Esse ordenamento das classes só acontece no caso de sistemas bi-partidos. Para sistemas compostos de três qubits já temos duas classes não equivalentes: as classes W e GHZ.

\subsubsection{Operações SLOCC e sistemas multi-partidos}

Para sistemas multi-partidos, um trabalho importante é o já citado Dür et al [17] onde eles obtêm a classificação SLOCC para sistemas compostos de três qubits. Outros resultados importantes são a classificação dos estados emaranhados de dimensionalidade $(2,2,3)$ e $(2,2,4)$ obtida por Miyake e Verstraete [31] e também a identificação de nove famílias não equivalentes por SLOCC de sistemas compostos de quatro qubits por Verstraete et al [30]. Depois disso, temos o método de Cornelio e Piza [39] que descreveremos em detalhe no Capítulo 3. Posteriormente, outros trabalhos foram desenvolvidos por Miyake [40], Chen et al [41, 42], Lamata et al [43, 44] e Li et al [45]. 
36 CAPÍTULO 2. TRANSFORMAÇÕES LOCAIS DOS ESTADOS EMARANHADOS 


\section{Capítulo 3}

\section{Emaranhamento tri-partido com um qubit}

No capítulo anterior, verificamos que o posto de Schmidt de um estado emaranhado multi-partido é uma medida do emaranhamento. Também vimos que o posto de Schmidt é invariante por SLOCC inversíveis. No entanto, no Capítulo 1, mostramos que uma decomposição do tipo Schmidt existe apenas para estados bastante particulares de sistemas compostos multi-partidos. Além disso, no caso multi-partido, encontrar as decomposições mínimas para um estado arbitrário, é um problema bastante difícil. Na maioria dos casos, o único método disponível é a solução de um complicado sistema de equações algébricas que, geralmente, admite apenas soluções numéricas.

Motivado pelo fato de que o posto de Schmidt de um estado multi-partido é invariante por SLOCC, neste capítulo, nós mostraremos como encontrar as decomposições mínimas de certos estados emaranhados tri-partidos com um qubit. Seremos capazes de resolver muitos casos, mas não todos. Nos casos em que o método a seguir funciona, seremos capazes de encontrar todas as decomposições do estado emaranhado com menos estados produtos que sucessivas decomposições de Schmidt. Chamaremos essas decomposições de sub-Schmidt. Seremos capazes de identificar diferentes famílias de estados, as quais terão diferentes decomposições sub-Schmidt. Para tanto, vamos introduzir o conceito de família de Jordan que será definido na seção 3.1 e é uma pequena variação do conceito de forma canônica de Jordan usual da algebra linear [47]. Dessa forma, mostraremos que as diferentes famílias de estados emaranhados corresponderão a diferentes famílias de Jordan e que, portanto, para obtermos as possíveis famílias de estados emaranhados, bastará listar as possíveis famílias de Jordan. Além disso, também mostraremos que um estado pertencente a uma determinada família não pode ser convertido em um estado pertencente a outra família por SLOCC. Também estabeleceremos condições necessárias e suficientes para que estados pertencentes à mesma família possam ser convertidos um no outro. 
Este capítulo é organizado da seguinte maneira. Na seção 3.1 descrevemos como obter as decomposições sub-Schmidt e o conceito de família de Jordan. Na seção 3.2 mostramos vários exemplos de estados com diferentes decomposições sub-Schmidt. Também mostramos alguns exemplos de estados para os quais o método apresentado não funciona. Na seção 3.3, mostramos que os estados emaranhados pertencentes a diferentes famílias de Jordan pertencem a diferentes classes de estados emaranhados por SLOCC. Também apresentamos um critério necessário e suficiente para determinarmos se dois estados pertencentes à mesma família estão relacionados por uma operação SLOCC. No caso deles estarem, também mostramos como é possível obter a operação que os relaciona. Na seção 3.4, discutimos brevemente as dificuldades em generalizar o método descrito na seção 3.1.

\subsection{Decomposições de estados emaranhados com um qubit}

Nesta seção, mostraremos como obter as decomposições sub-Schmidt de um estado emaranhado tri-partido com um qubit. Para entendermos a idéia geométrica que segue, primeiramente vamos lembrar como Dür et al [17] obtiveram que, para três qubits, existem estados emaranhados que podem ser decompostos em 2 ou 3 estados produto. Eles obtiveram esse resultado utilizando o resultado anterior de Sanpera et al [46] sobre planos em espaços produtos $C^{2} \otimes C^{2}$. Sanpera et al [46] haviam demonstrado que um plano em um espaço $C^{2} \otimes C^{2}$, gerado por dois estados emaranhados, ou possui dois estados produto ou possui apenas um. Esse resultado é importante porque, para um estado emaranhado $|\psi\rangle$ de dimensionalidade $(2,2,2)$, o suporte local de um subsistema composto de dois qubits é um plano $\mathcal{P}_{|\psi\rangle}$ contido em $C^{2} \otimes C^{2}$. Isso significa que podemos decompor $|\psi\rangle$ utilizando apenas estados contidos em $\mathcal{P}_{|\psi\rangle}$. E, se pudermos utilizar estados produto contidos em $\mathcal{P}_{|\psi\rangle}$ para decompormos $|\psi\rangle$, obteremos uma decomposição de $|\psi\rangle$ com menos estados produto.

Dessa forma, um estado emaranhado $|\psi\rangle$ de dimensionalidade $(2,2,2)$, pode ter, no seu suporte local de um subsistema de dois qubits, $\mathcal{P}_{|\psi\rangle}$, um ou dois estados produtos. No caso em que existem dois estados produto, é possível usar estes dois estados para decompormos $|\psi\rangle$ e, portanto, podemos decompor $|\psi\rangle$ em dois estados produto. Neste caso, $|\psi\rangle$ pertence à classe GHZ. No caso em que existe apenas um estado produto em $\mathcal{P}_{|\psi\rangle}$, precisamos de três estados produto para decompor $|\psi\rangle$ e portanto ele pertence à classe $\mathrm{W}$.

Assim sendo, estudaremos estados emaranhado tri-partidos de dimensionalidade $(n, n, 2)$. O suporte local nos dois sistemas de $n$ níveis será um plano, e nós vamos mostrar como encontrar os estados neste plano com posto de Schmidt menor que $n$. Uma maneira fácil de obtermos esse plano é usarmos uma decomposição qualquer em estados relativos conforme vimos na seção 1.1.2. Explicitamente, seja $\{|k\rangle\}_{k=0,1}$ uma base ortonormal qualquer para $C_{c}^{2}$, então temos 


$$
|\psi\rangle=\sum_{k=0,1} c_{k}\left|r_{k}\right\rangle|k\rangle,
$$

onde $\left|r_{k}\right\rangle \in C_{a}^{n} \otimes C_{b}^{n}$ é o estado relativo de $|k\rangle$ e $c_{k}$ é o coeficiente complexo apropriado da expansão de $|\psi\rangle$. Um vez que a dimensionalidade de $|\psi\rangle$ é $(n, n, 2)$, temos que o suporte local de $|\psi\rangle$ em $C_{a}^{n} \otimes C_{b}^{n}$ tem dimensão 2. Assim, os dois estados $\left|r_{k}\right\rangle$ 's são linearmente independentes e geram um plano $\mathcal{P}_{|\psi\rangle} \subset C_{a}^{n} \otimes C_{b}^{n}$ correspondente a este suporte local.

Agora que temos o plano $\mathcal{P}_{|\psi\rangle}$, vamos procurar os estados contidos neste que têm posto de Schmidt menor que $n$, pois com esses estados poderemos encontrar decomposições de $|\psi\rangle$ com menos estados produto que a dada na eq. (3.1). Além disso, sabemos que o posto de Schmidt é invariante por SLOCC e também é uma medida do emaranhamento, então estes estados serão centrais para entendermos as classificação por SLOCC dos estados tri-partidos. Esses estados podem ser encontrados considerando que qualquer estado $|\phi\rangle$ pertencente a $\mathcal{P}_{|\psi\rangle}$ pode ser escrito como combinação linear dos estados $\left|r_{k}\right\rangle$,

$$
|\phi\rangle=\alpha_{0}\left|r_{0}\right\rangle+\alpha_{1}\left|r_{1}\right\rangle
$$

onde $\alpha_{0}$ e $\alpha_{1}$ são coeficientes complexos. Para que não tenhamos que carregar a normalização de $|\phi\rangle$, nós ignoraremos esse vínculo entre $\alpha_{0}$ e $\alpha_{1}$. Obviamente, uma vez encontrado o estado $|\phi\rangle$ desejado, é sempre possível normalizá-lo. Da seção 1.1.2, sabemos que cada estado $|\phi\rangle$ pode ser visto como uma aplicação linear de $C_{a}^{n *}$ em $C_{b}^{n}$ (onde $C_{a}^{n *}$ é o espaço dual de $C_{a}^{n}$ ) definida através do produto escalar parcial de um estado qualquer do subsistema $S_{a},\left|u_{a}\right\rangle$, com $|\phi\rangle$ resultando em $\left\langle u_{a} \mid \phi\right\rangle \in C_{b}^{n}$. O posto de Schmidt desta aplicação linear é o posto de Schmidt do estado $|\phi\rangle$. Assim, para encontramos os estados $|\phi\rangle$ com posto de Schmidt menor que $n$, devemos procurar $\alpha_{0}$ e $\alpha_{1}$ tais que a equação

$$
\left\langleu _ { a } \left|\left(\alpha_{0}\left|r_{0}\right\rangle+\alpha_{1}\left|r_{1}\right\rangle\right)=0\right.\right.
$$

tenha pelo menos uma solução não trivial $\left\langle u_{a}\right| \in C_{a}^{n *}$.

É importante ressaltarmos que a questão relevante para nós é: quais são os $\alpha_{0}$ e $\alpha_{1}$ tal que exista algum estado $\left|u_{a}\right\rangle$ não nulo satisfazendo (3.3)?; e não quais são os estados $\left|u_{a}\right\rangle$ ? Apesar disso, o estado $\left|u_{a}\right\rangle$ tem uma interpretação física interessante. Vamos supor que temos um $\left|u_{a}\right\rangle$ não nulo para determinados valores de $\alpha_{0}$ e $\alpha_{1}$. Vamos supor também que fizemos uma medida no subsistema $S_{a}$ e obtemos que o estado do subsistema é exatamente $\left|u_{a}\right\rangle$. Nesse caso, embora o estado do subsistema $S_{a b}$ antes da medida seja misto (em particular ele pode ser visto como uma mistura de $\left|r_{0}\right\rangle$ e $\left.\left|r_{1}\right\rangle\right)$ obtemos que o estado de $S_{b}$ é também puro. Isso acontece porque a validade da equação (3.3) implica que $\alpha_{0}\left\langle u_{a} \mid r_{0}\right\rangle=$ $-\alpha_{1}\left\langle u_{a} \mid r_{1}\right\rangle$, isto é, os vetores $\left\langle u_{a} \mid r_{0}\right\rangle$ e $\left\langle u_{a} \mid r_{1}\right\rangle$ pertencentes a $C_{b}^{n}$ diferem apenas por um coeficiente complexo e definem um mesmo estado de $S_{b}$. Em termos mais físicos, o estado 
relativo de $\left|u_{a}\right\rangle$ é o mesmo se o estado de $S_{a b}$ for $\left|r_{0}\right\rangle$ ou $\left|r_{1}\right\rangle$ ou ainda qualquer outro estado contido em $\mathcal{P}_{|\psi\rangle}$. Se obtivermos 2 ou mais $\left|u_{a}\right\rangle$ 's linearmente independentes para os mesmos $\alpha_{0}$ e $\alpha_{1}$, teremos um subespaço de $\left|u_{a}\right\rangle$ 's contido em $C_{a}^{n}$ com o mesmo estado relativo para qualquer estado de $S_{a b}$ em $\mathcal{P}_{|\psi\rangle}$. Além disso, esse subespaço é o núcleo da aplicação linear de $C_{a}^{n *}$ em $C_{b}^{n}$ definida por estas combinações lineares particulares de $\left|r_{0}\right\rangle$ e $\left|r_{1}\right\rangle$ tal que $\alpha_{0}\left\langle u_{a} \mid r_{0}\right\rangle=-\alpha_{1}\left\langle u_{a} \mid r_{1}\right\rangle$. Dessa forma, essas combinações lineares terão posto de Schmidt $n$ menos a dimensão deste subespaço de $\left|u_{a}\right\rangle$ 's. A interpretação desses estados ficará mais clara nos exemplos da seção 3.2.

Para encontrarmos a solução da equação (3.3) vamos escolher uma base $\{|i\rangle\}$ em $C_{a}^{n}$ e uma base $\{|j\rangle\}$ em $C_{b}^{n}$. Assim podemos reescrever (3.3) como

$$
\sum_{i}\left\langle u_{a} \mid i j\right\rangle\left[\alpha_{0}\left\langle i j \mid r_{0}\right\rangle+\alpha_{1}\left\langle i j \mid r_{1}\right\rangle\right]=0
$$

Definindo as matrizes $R_{0}$ e $R_{1}$ com as componentes $\left[R_{0}\right]_{j i}=\left\langle i j \mid r_{0}\right\rangle$ e $\left[R_{1}\right]_{j i}=\left\langle i j \mid r_{1}\right\rangle$ e o vetor $u_{a}^{*}$ com as componentes $u_{a_{j}}^{*}=\left\langle u_{a} \mid i\right\rangle$, podemos obter uma forma matricial para eq. $(3.3)$,

$$
\left(\alpha_{0} R_{0}+\alpha_{1} R_{1}\right) u_{a}^{*}=0 .
$$

É importante notarmos que o posto de Schmidt do estado $|\phi\rangle=\alpha_{0}\left|r_{0}\right\rangle+\alpha_{1}\left|r_{1}\right\rangle$ é o posto da matriz $\left(\alpha_{0} R_{0}+\alpha_{1} R_{1}\right)$.

Vamos assumir agora que o estado $\left|r_{1}\right\rangle$ tem posto de Schmidt $n$, pois, como demonstraremos a seguir, dado que existe um estado deste tipo em $\mathcal{P}_{|\psi\rangle}$, quase todos os estados $\mathcal{P}_{|\psi\rangle}$ tem posto de Schmidt $n$ e apenas um número finito tem posto menor que $n$. Portanto, é sempre fácil encontrarmos um estado com posto de Schmidt $n$ em $\mathcal{P}_{|\psi\rangle}{ }^{1}$. Além disso, o resultado do método descrito não dependerá de qual estado com posto $n$ escolhermos. Dessa forma, $R_{1}$ é inversível, podemos reescrever (3.4) na forma de uma equação de auto-valores

$$
\left(R_{1}^{-1} R_{0}-\lambda\right) u_{a}^{*}=0
$$

onde $\left(\lambda=-\alpha_{1} / \alpha_{0}\right)$. É importante observar que $\lambda$ em (3.5) depende da razão dos coeficientes $\alpha_{1}$ e $\alpha_{0}$, os quais dependem da escolha da base em (3.1) para o plano $\mathcal{P}_{|\psi\rangle}$. No entanto, sabemos que o número de estados com posto de Schmidt menor que $n$ em $\mathcal{P}_{|\psi\rangle}$ não pode depender da base escolhida para $\mathcal{P}_{|\psi\rangle}$, assim como os respectivos postos de Schmidt.

Dessa forma, podemos nos perguntar o que mudaria na eq. (3.5) se tivéssemos escolhido uma outra base para $\mathcal{P}_{|\psi\rangle}$ na eq. (3.2). Vamos então chamar esta outra base de $\left\{\left|\phi_{0}\right\rangle,\left|\phi_{1}\right\rangle\right\}$,

\footnotetext{
${ }^{1}$ Em alguns casos não é possível encontrar um estado com posto de Schmidt $n$ em $\mathcal{P}_{|\psi\rangle}$, e é exatamente para estes casos que o método apresentado não funciona. Na seção 3.2.4, apresentamos um exemplo de um estado deste tipo. É por isso também que o método apresentado não funciona para estados de dimensionalidade $(n, m, 2)$ com $n \neq m$ (veja a seção 3.2.4).
} 
com

$$
\begin{aligned}
& \left|\phi_{0}\right\rangle=a\left|r_{0}\right\rangle+b\left|r_{1}\right\rangle \\
& \left|\phi_{1}\right\rangle=c\left|r_{0}\right\rangle+d\left|r_{1}\right\rangle
\end{aligned}
$$

onde $a, b, c$ e $d$ são coeficientes complexos e $(a d-b c)=1$. Nesse caso, como em (3.2), qualquer estado $|\phi\rangle$ em $\mathcal{P}_{|\psi\rangle}$ pode ser escrito como

$$
|\phi\rangle=\beta_{0}\left|\phi_{0}\right\rangle+\beta_{1}\left|\phi_{1}\right\rangle
$$

Para cada um dos estados $\left|\phi_{0}\right\rangle$ e $\left|\phi_{2}\right\rangle$ podemos associar uma matriz com as componentes $\left[\Phi_{0}\right]_{j i}=\left\langle i j \mid \phi_{0}\right\rangle$ e $\left[\Phi_{1}\right]_{j i}=\left\langle i j \mid \phi_{1}\right\rangle$. Podemos também supor que $\Phi_{1}$ é inversível. Assim, ao invés da eq. (3.5), obteríamos

$$
\left(\Phi_{1}^{-1} \Phi_{0}-\mu\right) u_{a}^{*}=0
$$

onde $\mu=-\beta_{1} / \beta_{0}$. Podemos nos perguntar agora quais são os aspectos comuns entre as matrizes $R_{1}^{-1} R_{0}$ e $\Phi_{1}^{-1} \Phi_{0}$ e como seus respectivos auto-valores $\lambda_{l}$ e $\mu_{l}$ se relacionam. Respondendo esta questão poderemos identificar os aspectos da eq. (3.5) que não dependem da escolha da base para $\mathcal{P}_{|\psi\rangle}$ definida em (3.1) e, portanto, correspondentes a aspectos invariantes do estado $|\psi\rangle$. De fato, veremos que (Teorema 3.1) os blocos de Jordan [47] das matrizes $R_{1}^{-1} R_{0}$ e $\Phi_{1}^{-1} \Phi_{0}$ na sua forma canônica têm a mesma dimensão embora os autovalores possam ser diferentes. Para deixarmos clara essa idéia, é conveniente definirmos o conceito de família de Jordan.

Definição 3.2 (família de Jordan): Diremos que duas matrizes $n$ por $n, A$ e $B$, pertencem à mesma família de Jordan se, para cada autovalor $\lambda_{l}$ de A, existe um autovalor $\mu_{l}$ de $B$ tal que os postos das matrizes $\left(A-\lambda_{l}\right)^{k} e\left(B-\mu_{l}\right)^{k}$ são iguais para todo inteiro positivo $k$.

O Teorema 3.1 a seguir nos garante que as matrizes $R_{1}^{-1} R_{0}$ e $\Phi_{1}^{-1} \Phi_{0}$ pertencem à mesma família de Jordan. Assim, ele permitirá obter uma classificação das famílias de estados emaranhados de dimensionalidade $(n, n, 2)$ em termos da enumeração das diferentes famílias de Jordan das matrizes quadradas de dimensão $n$.

Teorema 3.1: Sejam $R_{0}$ e $R_{1}$ duas matrizes $n$ por $n$ e $R_{1}$ inversivel. Sejam também $\Phi_{0}=a R_{0}+b R_{1}$ e $\Phi_{1}=c R_{0}+d R_{1}$ duas combinaçôes lineares de $R_{0}$ e $R_{1}$ tal que $\Phi_{1}$ também é inversivel e $(a d-b c)=1$. Então as matrizes $R_{1}^{-1} R_{0}$ e $\Phi_{1}^{-1} \Phi_{0}$ pertencem a mesma família de Jordan. Além disso, a relação entre os autovalores $\lambda_{l}$ de $R_{1}^{-1} R_{0}$ e $\mu_{l}$ de $\Phi_{1}^{-1} \Phi_{0}$ tal que

$$
\operatorname{posto}\left(R_{1}^{-1} R_{0}-\lambda_{l}\right)^{k}=\operatorname{posto}\left(\Phi_{1}^{-1} \Phi_{0}-\mu_{l}\right)^{k}
$$


é dada por

$$
\mu_{l}=\frac{a \lambda_{l}+b}{c \lambda_{l}+d}
$$

Prova: Vamos considerar primeiro o caso em que $c=0$. Então, como $(a d-b c)=1$, temos $a \neq 0, d \neq 0$ e

$$
\begin{aligned}
\left(\Phi_{1}^{-1} \Phi_{0}-\mu_{l}\right)^{k} & =\left[\left(d R_{1}\right)^{-1}\left(a R_{0}+b R_{1}\right)-\frac{a \lambda_{l}+b}{d}\right]^{k} \\
& =\left[\frac{b}{d}+\frac{a}{d} R_{1}^{-1} R_{0}-\frac{a \lambda_{l}+b}{d}\right]^{k} \\
& =\frac{a^{k}}{d^{k}}\left(R_{1}^{-1} R_{0}-\lambda_{l}\right)^{k}
\end{aligned}
$$

Logo $\operatorname{posto}\left(R_{1}^{-1} R_{0}-\lambda_{l}\right)^{k}=\operatorname{posto}\left(\Phi_{1}^{-1} \Phi_{0}-\mu_{l}\right)^{k}$.

No caso em que $c \neq 0$, devemos ter também $c \lambda_{l}+d \neq 0$, pois $c \lambda_{l}+d$ é um autovalor de $\Phi_{1}$ que é inversível. Nesse caso devemos notar que

$$
\begin{aligned}
\Phi_{1}^{-1} \Phi_{0} & =\left(c R_{0}+d R_{1}\right)^{-1}\left(a R_{0}+b R_{1}\right) \\
& =\left[R_{1}\left(c R_{1}^{-1} R_{0}+d\right)\right]^{-1} R_{1}\left(a R_{1}^{-1} R_{0}+b\right) \\
& =\left(c R_{1}^{-1} R_{0}+d\right)^{-1}\left(a R_{1}^{-1} R_{0}+b\right) .
\end{aligned}
$$

Devemos notar também que dividindo o polinômio $a z+b$ por $c z+d$ obtemos dois valores complexos $\gamma$ e $\delta$ tais que

$$
a z+b=\gamma(c z+d)+\delta
$$

onde zé um número complexo arbitrário. Dessa forma, temos que

$$
a R_{1}^{-1} R_{0}+b=\gamma\left(c R_{1}^{-1} R_{0}+d\right)+\delta
$$

e, portanto,

$$
\begin{aligned}
\Phi_{1}^{-1} \Phi_{0} & =\left(c R_{1}^{-1} R_{0}+d\right)^{-1}\left[\gamma\left(c R_{1}^{-1} R_{0}+d\right)+\delta\right] \\
& =\gamma+\delta\left(c R_{1}^{-1} R_{0}+d\right)^{-1}
\end{aligned}
$$

Então,

$$
\begin{aligned}
\left(\Phi_{1}^{-1} \Phi_{0}-\mu_{l}\right)^{k} & =\left(\gamma+\delta\left(c R_{1}^{-1} R_{0}+d\right)^{-1}-\gamma-\frac{\delta}{c \lambda_{l}+d}\right)^{k} \\
& =\delta^{k} c^{k}\left(c \lambda_{l}+d\right)^{-k}\left(c R_{1}^{-1} R_{0}+d\right)^{-k}\left(\lambda_{l}-R_{1}^{-1} R_{0}\right)^{k}
\end{aligned}
$$


Como $\Phi_{0}$ e $\Phi_{1}$ são linearmente independentes, temos $\delta \neq 0$. Além disso, como $\Phi_{1}$ é inversível, temos $c \lambda_{l}+d \neq 0$, pois é um autovalor de $\Phi_{1}$. A matriz $\left(c R_{1}^{-1} R_{0}+d\right)$ também é inversível, pois é igual a $R_{1}^{-1} \Phi_{1}$. Logo segue que $\operatorname{posto}\left(R_{1}^{-1} R_{0}-\lambda_{l}\right)^{k}=\operatorname{posto}\left(\Phi_{1}^{-1} \Phi_{0}-\mu_{l}\right)^{k}$.

Dessa forma, para cada autovalor $\lambda_{l}$ encontrado usando uma base $\left\{\left|r_{0}\right\rangle,\left|r_{1}\right\rangle\right\}$, existirá um autovalor correspondente $\mu_{l}$ em outra base $\left\{\left|\phi_{0}\right\rangle,\left|\phi_{1}\right\rangle\right\}$. Além disso, o posto da matriz $\left(R_{1}^{-1} R_{0}-\lambda_{l}\right)^{k}$ é igual ao posto da matriz $\left(\Phi_{1}^{-1} \Phi_{0}-\mu_{l}\right)^{k}$. Para cada autovalor $\lambda_{l}$ ou $\mu_{l}$, podemos associar um estado $\left|\xi_{l}\right\rangle$ contido em $\mathcal{P}_{|\psi\rangle}$ definido por

$$
\left|\xi_{l}\right\rangle=\left|r_{0}\right\rangle-\lambda_{l}\left|r_{l}\right\rangle=\left|\phi_{0}\right\rangle-\mu_{l}\left|\phi_{1}\right\rangle
$$

Assim, o posto das matrizes $\left(R_{1}^{-1} R_{0}-\lambda_{l}\right)$ e $\left(\Phi_{1}^{-1} \Phi_{0}-\mu_{l}\right), k=1$, é simplesmente o posto de Schmidt do estado $\left|\xi_{l}\right\rangle$ e, portanto, é uma característica independente do plano $\mathcal{P}_{|\psi\rangle}$, no qual este está contido, ou do estado emaranhado tri-partido $|\psi\rangle$ do qual o obtemos. Porém, isso não é verdade para os postos das matrizes $\left(R_{1}^{-1} R_{0}-\lambda_{l}\right)^{k}$ e $\left(\Phi_{1}^{-1} \Phi_{0}-\mu_{l}\right)^{k}$ para $k \geq 2$. Nesse caso, estes postos podem ser diferentes para um mesmo estado $\left|\xi_{l}\right\rangle$ contido em planos distintos em $C_{a}^{n} \otimes C_{b}^{n}$. Dessa forma, devemos entender estes postos, para $k \geq 2$, como propriedades invariantes do estado $\left|\xi_{l}\right\rangle$ quando contido no plano $\mathcal{P}_{|\psi\rangle}$, isto é, como uma propriedade que só pode ser entendida considerando o estado tri-partido $|\psi\rangle$ do qual $\mathcal{P}_{|\psi\rangle}$ é obtido. A distinção entre estes postos para $k \geq 2$ é importante para a classificação das famílias de Jordan para $n \geq 4$. Na seção 3.2.3, através de exemplos, descrevemos essas diferenças para estados emaranhados de dimensionalidade $(4,4,2)$. Esses exemplos ilustrarão como relacionamos as diferentes famílias de estados emaranhados com as respectivas famílias de Jordan e como podemos interpretar a dimensão dos blocos de Jordan em termos de correlações quânticas entre os subsistemas da Alice e Bob.

Devemos observar também que esses resultados não dependem da ordem dos subsistemas. Se trocássemos o papel dos subsistemas no procedimento descrito até agora, teríamos que os estados $\left|r_{0}\right\rangle$ e $\left|r_{1}\right\rangle$ na eq. (3.3) deveriam ser entendidos como aplicações lineares de $C_{b}^{n *}$ em $C_{a}^{n}$. Ao invés das matrizes $R_{0}$ e $R_{1}$ na eq. (3.4), obteríamos suas respectivas matrizes transpostas $R_{0}^{T}$ e $R_{1}^{T}$. Portanto, ao invés da matriz $R_{1}^{-1} R_{0}$ na eq. (3.5), obteríamos a matriz $\left(R_{0} R_{1}^{-1}\right)^{T}$, a qual é similar a $R_{1}^{-1} R_{0}$ e então tem a mesma forma canônica de Jordan [47].

Para obtermos uma decomposição de $|\psi\rangle$ em termos de um número menor de estados produto, podemos agora utilizar a eq. (3.5) para obtermos os estados $\left|\xi_{l}\right\rangle$ com posto de Schmidt menor que $n$. Podemos encontrar um estado $\left|\xi_{l}\right\rangle$ para cada autovalor $\lambda_{l}$ de $R_{1}^{-1} R_{0}$ na eq. (3.5). Explicitamente, vamos supor que encontramos duas soluções da eq. (3.5) e, portanto, temos dois estados normalizados $\left|\xi_{1}\right\rangle$ e $\left|\xi_{2}\right\rangle$ pertencentes a $\mathcal{P}_{|\psi\rangle}$ com posto de Schmidt menor que $n$. Como os estados $\left|\xi_{1}\right\rangle$ e $\left|\xi_{2}\right\rangle$ geram $\mathcal{P}_{|\psi\rangle}$, temos que $|\psi\rangle$ está contido 
no subespaço de $C_{a}^{n} \otimes C_{b}^{n} \otimes C_{c}^{2}$ gerado pela base

$$
\left\{\left|\xi_{1}\right\rangle \otimes|0\rangle,\left|\xi_{1}\right\rangle \otimes|1\rangle,\left|\xi_{2}\right\rangle \otimes|0\rangle,\left|\xi_{2}\right\rangle \otimes|1\rangle\right\}
$$

É importante ressaltarmos que $\left|\xi_{1}\right\rangle$ e $\left|\xi_{2}\right\rangle$ geralmente não são ortogonais e, portanto, os elementos da base (3.7) não são todos ortogonais entre si. Expandindo $|\psi\rangle$ na base (3.7), temos

$$
\begin{aligned}
|\psi\rangle & =\alpha_{1}\left|\xi_{1}\right\rangle \otimes|0\rangle+\alpha_{2}\left|\xi_{1}\right\rangle \otimes|1\rangle+\alpha_{3}\left|\xi_{2}\right\rangle \otimes|0\rangle+\alpha_{4}\left|\xi_{2}\right\rangle \otimes|1\rangle \\
& =\left|\xi_{1}\right\rangle\left|c_{1}\right\rangle+\left|\xi_{2}\right\rangle\left|c_{2}\right\rangle,
\end{aligned}
$$

onde $\alpha_{i}, i=1,2,3,4$, são os respectivos coeficientes da expansão e

$$
\begin{aligned}
& \left|c_{1}\right\rangle=\alpha_{1}|0\rangle+\alpha_{2}|1\rangle \\
& \left|c_{2}\right\rangle=\alpha_{3}|0\rangle+\alpha_{4}|1\rangle
\end{aligned}
$$

É importante observarmos que os estados $\left|c_{1}\right\rangle$ e $\left|c_{2}\right\rangle$ geralmente também não são ortogonais e não estão normalizados. Da eq. (3.8), podemos ver que $|\psi\rangle$ pode ser decomposto em termos de um número de estados produto dado pela soma dos postos de Schmidt dos estados $\left|\xi_{1}\right\rangle$ e $\left|\xi_{2}\right\rangle$. Além disso, devemos lembrar que a eq. (3.5) pode ter de 1 a $n$ autovalores distintos. No caso de um único autovalor, temos apenas um estado com posto de Schmidt menor que $n$ e temos que escolher algum outro estado em $\mathcal{P}_{|\psi\rangle}$ com posto de Schmidt $n$. No caso em que a eq. (3.5) tem $m$ soluções, teremos $\left(\begin{array}{c}m \\ 2\end{array}\right)$ combinações distintas de dois estados $\left|\xi_{i}\right\rangle$ e $\left|\xi_{j}\right\rangle, i \neq j$ e $i, j=1,2, \ldots, m$, para escrevermos $|\psi\rangle$ na eq. (3.8). Evidentemente, é sempre possível escolhermos um estado com posto de Schmidt menor que $n$ e outro com posto de Schmidt $n$ e, também, cada $\left|\xi_{i}\right\rangle$ possui infinitas decomposições em estados relativos. Assim, sempre existem infinitas decomposições sub-Schmidt para $|\psi\rangle$ na eq. (3.8). Todas essas decomposições terão menos que $2 n$ estados produto que teríamos utilizando duas decomposições de Schmidt sucessivas.

Dessa forma, para cada uma das possíveis famílias de Jordan da matriz $R_{1}^{-1} R_{0}$, podemos associar uma família de estados emaranhados $|\psi\rangle$. Esses estados terão todos dimensionalidade $(n, n, 2)$. A única exceção é o caso das familias cuja matriz $R_{1}^{-1} R_{0}$ é proporcional à identidade, pois isso implica que os estados $\left|r_{0}\right\rangle$ e $\left|r_{1}\right\rangle$ são idênticos e portanto o qubit não está emaranhado com os outros dois subsistemas $n$-dimensionais. De fato, nesse caso temos um estado emaranhamento puro bi-partido no subsistema $S_{a b},\left|r_{0}\right\rangle=\left|r_{1}\right\rangle$, com posto de Schmidt $n$ e o qubit não está emaranhado com os outros subsistemas $n$-dimensionais.

Na próxima seção discutimos alguns exemplos de decomposições sub-Schmidt e suas respectivas famílias de Jordan. Também discutimos qual o significado dos postos das matrizes $\left(R_{1}^{-1} R_{0}-\lambda_{l}\right)^{k}$ nos exemplos de estados com dimensionalidade $(4,4,2)$, seção 
3.2.3. Na seção 3.3 demonstramos que todas essas propriedades são invariantes através de SLOCC.

\subsection{Exemplos}

\subsubsection{Exemplos: Três qubits.}

As propriedades dos estados emaranhados de três qubits foram estudadas primeiramente por Dür et al [17]. Nestes exemplos, reproduziremos esses resultados usando os métodos da seção 3.1. Na seção 3.3, também utilizamos nossos métodos para reproduzirmos o resultado de Dür et al [17] de que existem duas classes não equivalentes de estados emaranhados de três qubits por SLOCC.

Um estado $|\psi\rangle$ com dimensionalidade $(2,2,2)$ pode ser identificado com a família de Jordan de uma das seguintes matrizes

$$
W=\left(\begin{array}{cc}
\lambda_{1} & 1 \\
0 & \lambda_{1}
\end{array}\right) \quad G H Z=\left(\begin{array}{cc}
\lambda_{1} & 0 \\
0 & \lambda_{2}
\end{array}\right)
$$

onde $\lambda_{1} \neq \lambda_{2}$. Como veremos a seguir, as famílias de Jordan das matrizes $\mathrm{W}$ e GHZ correspondem as classes de estados emaranhados W e GHZ definidas por Dür et al [17].

\section{Família W}

Vamos supor que temos um estado $\left|\psi_{W}\right\rangle$ pertencente a família de Jordan da matriz W. Assim, o suporte local de $\left|\psi_{W}\right\rangle$ no subsistema $S_{a b}$, o plano $\mathcal{P}_{\left|\psi_{W}\right\rangle}$, contem apenas um estado

$$
\left|\xi_{\lambda_{1}}\right\rangle=\left|r_{0}\right\rangle-\lambda_{1}\left|r_{1}\right\rangle
$$

com posto de Schmidt 1. Ou seja, $\left|\xi_{\lambda_{1}}\right\rangle$ é o único estado não emaranhado no plano $\mathcal{P}_{\left|\psi_{W}\right\rangle}$. Neste caso, se quisermos gerar o plano $\mathcal{P}_{\left|\psi_{W}\right\rangle}$, precisamos escolher um outro estado $\left|\phi_{0}\right\rangle \in$ $\mathcal{P}_{\left|\psi_{W}\right\rangle}$ com posto de Schmidt 2 como $\left|\xi_{2}\right\rangle$ na eq. (3.8). Desta eq. (3.8), obtemos que qualquer estado desta família pode ser escrito como uma superposição de três estados produto. Como vimos na seção 2.2.3, os estados de três qubits que necessitam de uma superposição de três produtos são ditos pertencentes à classe W [17].

O exemplo característico desta classe é o estado

$$
|W\rangle=\frac{1}{\sqrt{3}}(|00\rangle|1\rangle+(|01\rangle+|10\rangle)|0\rangle) .
$$

Para este estado temos que $\left|\xi_{\lambda_{1}}\right\rangle=|00\rangle$ e $\left|\xi_{2}\right\rangle=(|01\rangle+|10\rangle)$. Neste caso, temos $\left\langle u_{a}\right|=\left\langle 1_{a}\right|$ na eq. (3.5) e $\left\langle 1_{a}\right|$ gera o núcleo da aplicação linear definida por $\left|\xi_{\lambda_{1}}\right\rangle$ de $C_{a}^{2 *}$ em $C_{b}^{2}$, pois $\left\langle 1_{a} \mid 00\right\rangle=0$. Dessa forma, se em uma observação encontrarmos o subsistema $S_{a}$ no estado 
$|1\rangle$, teremos que o estado do sistema composto $S_{a b c}$ reduz-se ao estado puro separável igual $|100\rangle$.

Para mostrarmos que o estado $|W\rangle$ pertence a família de Jordan da matriz $W$ e não possui nenhuma decomposição em apenas dois estados produtos, precisamos de dois estados que gerem o plano $P_{|W\rangle}$, tal que pelo menos um deles tem posto de Schmidt 2. Podemos usar os estados $\left|\xi_{\lambda_{1}}\right\rangle=|00\rangle$ e $\left|\xi_{2}\right\rangle=(|01\rangle+|10\rangle)$ que são facilmente obtidos de (3.9) e correspondem as matrizes

$$
\Xi_{\lambda_{1}}=\left(\begin{array}{cc}
1 & 0 \\
0 & 0
\end{array}\right) \quad \text { e } \quad \Xi_{2}=\left(\begin{array}{cc}
0 & 1 \\
1 & 0
\end{array}\right)
$$

em (3.4). Dessa forma, temos

$$
\Xi_{2}^{-1} \Xi_{\lambda_{1}}=\left(\begin{array}{ll}
0 & 1 \\
1 & 0
\end{array}\right)\left(\begin{array}{ll}
1 & 0 \\
0 & 0
\end{array}\right)=\left(\begin{array}{ll}
0 & 0 \\
1 & 0
\end{array}\right)
$$

que é similar a $W \operatorname{com} \lambda_{1}=0$.

\section{Família GHZ}

No caso de um estado pertencente à família GHZ, é possível encontrarmos dois estados não emaranhados no suporte local do subsistema $S_{a b}, \mathcal{P}_{G H Z}$. Para cada um dos dois autovalores $\lambda_{l}$, temos um estado

$$
\left|\xi_{\lambda_{l}}\right\rangle=\left|r_{0}\right\rangle-\lambda_{l}\left|r_{1}\right\rangle
$$

onde $l=1,2$. Dessa forma, podemos utilizar estes dois estados para gerar $\mathcal{P}_{G H Z}$ e podemos escrever qualquer estado desta família como uma superposição de dois estados produto. Estados que podem ser decompostos com dois estados produtos pertencem à classe GHZ [17].

O exemplo característico desta classe é o estado

$$
|G H Z\rangle=\frac{1}{\sqrt{2}}(|000\rangle+|111\rangle)
$$

Para este estado temos $\left|\xi_{\lambda_{1}}\right\rangle=|00\rangle$, para o qual temos o núcleo gerado por $\left\langle u_{a_{\lambda_{1}}}\right|=\left\langle 1_{a}\right|$. Também temos $\left|\xi_{\lambda_{2}}\right\rangle=|11\rangle$, para o qual temos o núcleo gerado por $\left\langle u_{a_{\lambda_{2}}}\right|=\left\langle 0_{a}\right|$.

Para mostrarmos que o estado $|G H Z\rangle$ pertence à família de Jordan da matriz GHZ, podemos escolher os estados $|00\rangle$ e $|11\rangle$ como geradores do plano $\mathcal{P}_{|G H Z\rangle}$. Esses dois estados correspondem às matrizes 


$$
\Xi_{\lambda_{1}}=\left(\begin{array}{cc}
1 & 0 \\
0 & 0
\end{array}\right) \quad \text { e } \quad \Xi_{\lambda_{2}}=\left(\begin{array}{cc}
0 & 0 \\
0 & 1
\end{array}\right),
$$

respectivamente. Como tanto $\Xi_{\lambda_{1}}$ quanto $\Xi_{\lambda_{2}}$ não são inversíveis, precisamos encontrar um combinação linear delas que seja inversível. Escolhemos

$$
\Phi_{1}=\Xi_{\lambda_{1}}+\Xi_{\lambda_{2}}=\left(\begin{array}{cc}
1 & 0 \\
0 & 1
\end{array}\right)
$$

e temos

$$
\Phi_{1}^{-1} \Xi_{\lambda_{1}}=\left(\begin{array}{cc}
1 & 0 \\
0 & 0
\end{array}\right)
$$

que é similar a matriz GHZ $\operatorname{com} \lambda_{1}=1$ e $\lambda_{2}=0$.

\section{Observação}

É importante salientarmos que nosso método fornece uma maneira prática, a qual é uma alternativa ao método proposto por Dür et al [17], de decidirmos se um dado estado pertence à classe W ou à classe GHZ. A dimensionalidade de um estado emaranhado $|\psi\rangle$ pode ser determinada facilmente através do determinante das matrizes densidade reduzidas. Uma vez determinado que a dimensionalidade do emaranhamento de $|\psi\rangle$ é $(2,2,2)$, basta verificarmos se a eq. (3.5) tem uma ou duas soluções. Com mais alguns cálculos simples é possível obtermos as decomposições sub-Schmidt do estado $|\psi\rangle$. Também veremos na seção 3.3 que é possível determinarmos construtivamente as operações do tipo SLOCC que transformariam este estado $|\psi\rangle$ nos estados $|W\rangle$ ou $|G H Z\rangle$, dependendo da classe à qual ele pertença. Todos os outros estados com dimensionalidade de emaranhamento menor que $(2,2,2)$ (onde menor significa que pelo menos um dos suportes locais é de menor dimensão e nenhum é maior) correspondem a estados emaranhados bi-partidos, nos quais, pelo menos um dos subsistemas não está emaranhado com os outros ou a um estado produto.

\subsubsection{Exemplos: dois sistemas de 3 níveis e um qubit}

Nesse exemplo, veremos novas famílias de estados emaranhados, as quais são simples de serem obtidas e exemplificam bem os métodos descritos na seção 3.1. No entanto, alguns aspectos dos estados emaranhados tri-partidos com um qubit ainda não aparecem em estados desta dimensionalidade. O significado da dimensão dos blocos de Jordan de cada família aparecerá nos exemplos da próxima seção 3.2.3.

Para estados emaranhados de dimensionalidade $(3,3,2)$, podemos listar cinco famílias 
de Jordan distintas correspondentes às matrizes

$$
\begin{array}{rlrl}
A=\left(\begin{array}{ccc}
\lambda_{1} & 1 & 0 \\
0 & \lambda_{1} & 1 \\
0 & 0 & \lambda_{1}
\end{array}\right) & B=\left(\begin{array}{ccc}
\lambda_{1} & 0 & 0 \\
0 & \lambda_{1} & 1 \\
0 & 0 & \lambda_{1}
\end{array}\right) & C=\left(\begin{array}{ccc}
\lambda_{1} & 0 & 0 \\
0 & \lambda_{2} & 1 \\
0 & 0 & \lambda_{2}
\end{array}\right) \\
D=\left(\begin{array}{ccc}
\lambda_{1} & 0 & 0 \\
0 & \lambda_{2} & 0 \\
0 & 0 & \lambda_{2}
\end{array}\right) & E=\left(\begin{array}{ccc}
\lambda_{1} & 0 & 0 \\
0 & \lambda_{2} & 0 \\
0 & 0 & \lambda_{3}
\end{array}\right)
\end{array}
$$

onde $\lambda_{l} \neq \lambda_{l^{\prime}}$ para $l \neq l^{\prime}$. Na seqüência, construiremos exemplos de estados pertencentes a cada uma dessas famílias. Para cada um dos estados, também mostramos um diagrama das correlações existentes entre os subsistemas $S_{a}$ e $S_{b}$. Esses diagramas são baseados nos estados $\left|\xi_{l}\right\rangle$ utilizados para escrever a decomposição sub-Schmidt do estado emaranhado tri-partido $|\psi\rangle$ e nos ajudam a ter uma idéia intuitiva das correlações quânticas e do significado da invariância das famílias de Jordan através de transformações SLOCC. No caso da Família A, exemplificamos em mais detalhes como construir o diagrama a partir da decomposição sub-Schmidt do estado $|\psi\rangle$.

\section{Família A}

A maneira mais fácil de obtermos estados emaranhados para cada família é escolhermos o suporte local no subsistema $S_{a b}$ como gerado por estados cujas matrizes sejam as desejadas.

Para construirmos um exemplo de um estado emaranhado $\left|\psi_{A}\right\rangle$ pertencente à família A, vamos escolher dois estados $\left|\phi_{0}\right\rangle$ e $\left|\phi_{1}\right\rangle$ tais que suas respectivas matrizes sejam

$$
\Phi_{0}=\left(\begin{array}{ccc}
\lambda_{1} & 1 & 0 \\
0 & \lambda_{1} & 1 \\
0 & 0 & \lambda_{1}
\end{array}\right) \quad \text { e } \quad \Phi_{1}=\left(\begin{array}{ccc}
1 & 0 & 0 \\
0 & 1 & 0 \\
0 & 0 & 1
\end{array}\right) .
$$

Assim, evidentemente temos $\Phi_{1}^{-1} \Phi_{0}=A$ e assumimos que $\left|\phi_{0}\right\rangle$ e $\left|\phi_{1}\right\rangle$ pertencem e determinam o suporte local do estado $\left|\psi_{A}\right\rangle$ no subsistema $S_{a b}, \mathcal{P}_{\left|\psi_{A}\right\rangle}$. Então, das eq. (3.3) e (3.4), obtemos que o estado

$$
\left|\xi_{1}\right\rangle=\left|\phi_{0}\right\rangle-\lambda_{1}\left|\phi_{1}\right\rangle=|10\rangle+|21\rangle
$$

possui posto de Schmidt 2. Como $\lambda_{1}$ é o único autovalor nesta família, da mesma forma que para um estado na classe $\mathrm{W},\left|\xi_{1}\right\rangle$ é o único estado em $\mathcal{P}_{\left|\psi_{A}\right\rangle}$ com posto de Schmidt menor que 3 e precisamos escolher um outro estado pertencente a $\mathcal{P}_{\left|\psi_{A}\right\rangle}$ com posto de Schmidt 3 como $\left|\xi_{2}\right\rangle$. Escolhemos $\left|\xi_{2}\right\rangle=\left|\phi_{1}\right\rangle$. Dessa forma, da eq. (3.8), obtemos que 


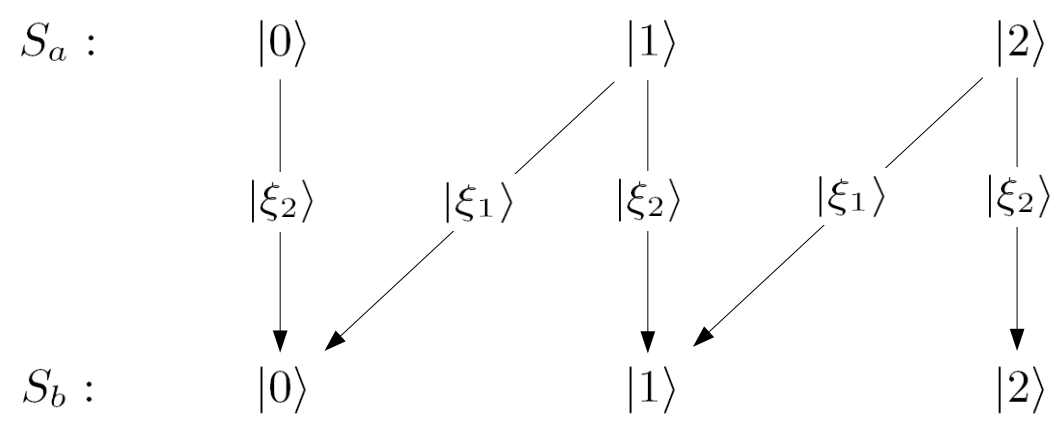

Figura 3.1: Diagrama de correlações quânticas dos estados $\left|\xi_{1}\right\rangle$ e $\left|\xi_{2}\right\rangle$ representados nas suas bases de Schmidt para o estado $\left|\psi_{A}\right\rangle$. Observando o diagrama, fica claro que apenas o estado $|0\rangle$ tem um correlação bem definida de $S_{a}$ para $S_{b}$. No entanto, qualquer estado do subespaço $\{|1\rangle,|2\rangle\}$ de $C_{a}^{3}$ não está correlacionado com um único estado em $C_{b}^{3}$, mas sim com um estado misto que corresponde a mistura das correlações de $\left|\xi_{1}\right\rangle$ e $\left|\xi_{2}\right\rangle$.

$\left|\psi_{A}\right\rangle$ pode ser escrito como uma superposição de cinco estados produto,

$$
\left|\psi_{A}\right\rangle=\frac{1}{\sqrt{5}}[(|10\rangle+|21\rangle)|0\rangle+(|00\rangle+|11\rangle+|22\rangle)|1\rangle] .
$$

Na Figura 3.1, podemos visualizar como as correlações entre os subsistemas $S_{a}$ e $S_{b}$, dados por $\left|\xi_{1}\right\rangle$ e $\left|\xi_{2}\right\rangle$, se relacionam de uma forma "cruzada". Para construirmos esse diagramas escolhemos as correlações estabelecidas pelas bases de Schmidt de $\left|\xi_{1}\right\rangle$ e $\left|\xi_{2}\right\rangle$. Assim é importante, ao interpretarmos a Figura 3.1, que estamos ilustrando as correlações em uma determinada base. Além disso, escolhemos $\left|\psi_{A}\right\rangle$ de forma tal que $\left|\xi_{1}\right\rangle$ e $\left|\xi_{2}\right\rangle$ tenha as mesmas bases de Schmidt. Essa é uma característica um tanto particular de $\left|\psi_{A}\right\rangle$ e não é verdadeira para qualquer estado desta e das outras famílias que apresentaremos. No entanto, o fato de que existe apenas um estado no subsistema $S_{a},|0\rangle$, tal que, no caso de Alice verificar que o subsistema $S_{a}$ encontra-se neste estado, o estado de $S_{b}$ resulta também em um estado puro é independente desta escolha da base. É, portanto, uma característica dos estados desta família.

Nesse sentido, esses diagrams que apresentaremos para os estados nos ajudarão a identificar essas características e a relaciona-las com as respectivas famílias de Jordan dos estados.

\section{Família B}

Similarmente ao exemplo anterior, vamos escolher dois estados que geram um plano $\mathcal{P}_{\left|\psi_{B}\right\rangle}$ que corresponda ao suporte local de um estado $\left|\psi_{B}\right\rangle$ pertencente a família da matriz B. Escolhemos $\left|\phi_{0}\right\rangle$ e $\left|\phi_{1}\right\rangle$ tais que estes correspondam respectivamente às seguintes 


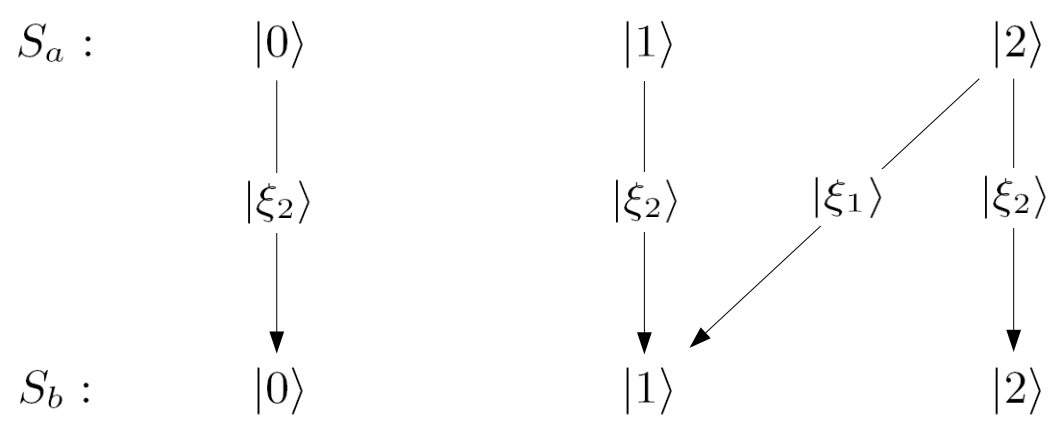

Figura 3.2: Diagrama de correlações do estado $\left|\psi_{B}\right\rangle$. O subespaço gerado por $\{|0\rangle,|1\rangle\}$ de $C_{a}^{3}$ tem uma correlação bem definida de $S_{a}$ para $S_{b}$ dada pelo estado $\left|\xi_{2}\right\rangle$.

matrizes

$$
\Phi_{0}=\left(\begin{array}{ccc}
\lambda_{1} & 0 & 0 \\
0 & \lambda_{1} & 1 \\
0 & 0 & \lambda_{1}
\end{array}\right) \quad \text { e } \quad \Phi_{1}=\left(\begin{array}{ccc}
1 & 0 & 0 \\
0 & 1 & 0 \\
0 & 0 & 1
\end{array}\right)
$$

Neste caso existe um estado $\left|\xi_{1}\right\rangle$ com posto de Schmidt 1 em $\mathcal{P}_{\left|\psi_{B}\right\rangle}$,

$$
\left|\xi_{1}\right\rangle=\left|r_{0}\right\rangle-\lambda_{1}\left|r_{1}\right\rangle=|21\rangle
$$

Assim, também precisamos escolher um estado pertencente a $\mathcal{P}_{\left|\psi_{B}\right\rangle}$ com posto de Schmidt 3 como $\left|\xi_{2}\right\rangle$, o qual tomaremos $\left|\xi_{2}\right\rangle=\left|\phi_{1}\right\rangle$. Da eq. (3.8), obtemos então que $\left|\psi_{B}\right\rangle$ pode ser escrito como uma superposição de quatro estados produto,

$$
\left|\psi_{B}\right\rangle=\frac{1}{2}[|21\rangle|0\rangle+(|00\rangle+|11\rangle+|22\rangle)|1\rangle]
$$

Note que este é o primeiro caso encontrado em que $\left|\xi_{1}\right\rangle$ tem como núcleo um subespaço bi-dimensional gerado pelos estados $\left\langle 0_{a}\right|$ e $\left\langle 1_{a}\right|$. Assim, se uma observação de Alice no subsistema $S_{a}$ resultar em qualquer estado deste subespaço, o estado $\left|\psi_{B}\right\rangle$ é reduzido a um estado produto. Esse aspecto pode ser melhor observado no diagrama da Figura 3.2.

\section{Família C}

Para esta família, é conveniente escolhermos diretamente os estados com posto de Schmidt menor que 3 em $\mathcal{P}_{\left|\psi_{C}\right\rangle}$ para depois mostrarmos que eles correspondem a um estado tri-partido $\left|\psi_{C}\right\rangle$ pertencente à família C. Se olharmos para a família de Jordan deste estado, podemos verificar que existem dois estados com posto de Schmidt 2 no suporte local de $\left|\psi_{C}\right\rangle$ no subsistema $S_{a b}, \mathcal{P}_{\left|\psi_{C}\right\rangle}$. Escolhemos então os estados

$$
\left|\xi_{1}\right\rangle=|11\rangle+|22\rangle
$$




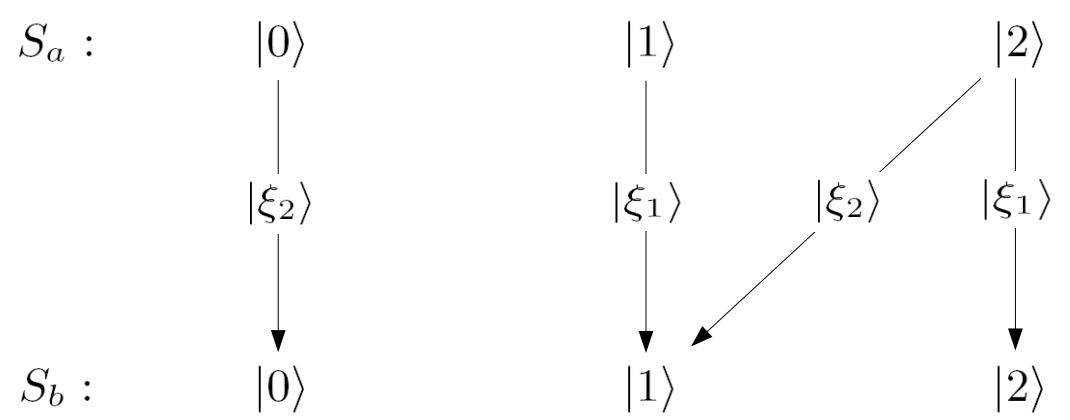

Figura 3.3: Diagrama de correlações do estado $\left|\psi_{c}\right\rangle$. Os estados $|0\rangle$ e $|1\rangle$ tem correlações bem definidas pelos estados $\left|\xi_{2}\right\rangle$ e $\left|\xi_{1}\right\rangle$, respectivamente, de $S_{a}$ para $S_{b}$.

e

$$
\left|\xi_{2}\right\rangle=|00\rangle+|21\rangle
$$

Utilizando esses dois estados, $\left|\psi_{C}\right\rangle$ pode ser escrito como uma superposição de quatro estados produto,

$$
\left|\psi_{c}\right\rangle=\frac{1}{2}[(|00\rangle+|21\rangle)|0\rangle+(|11\rangle+|22\rangle)|1\rangle]
$$

A Figura 3.3 mostra o diagrama de correlações para o estado $\left|\psi_{C}\right\rangle$.

Para demonstrarmos que $\left|\psi_{C}\right\rangle$ é um estado pertencente a família C, precisamos de um estado com posto de Schmidt $3 \mathrm{em} \mathcal{P}_{\left|\psi_{C}\right\rangle}$. As respectivas matrizes dos estados $\left|\xi_{1}\right\rangle$ e $\left|\xi_{2}\right\rangle$ são

$$
\Xi_{1}=\left(\begin{array}{ccc}
0 & 0 & 0 \\
0 & 1 & 0 \\
0 & 0 & 1
\end{array}\right) \quad \text { e } \quad \Xi_{2}=\left(\begin{array}{ccc}
1 & 0 & 0 \\
0 & 0 & 1 \\
0 & 0 & 0
\end{array}\right) .
$$

Então, a matriz

$$
\Phi_{1}=\Xi_{1}+\Xi_{2}=\left(\begin{array}{ccc}
1 & 0 & 0 \\
0 & 1 & 1 \\
0 & 0 & 1
\end{array}\right)
$$

é inversível e corresponde a um estado com posto de Schmidt 3 em $\mathcal{P}_{\left|\psi_{C}\right\rangle}$. Dessa forma, podemos tomar as matrizes $\Phi_{1}$ e $\Xi_{2}$ como as matrizes $R_{1}$ e $R_{0}$ na eq. (3.4) e

$$
\Phi_{1}^{-1} \Xi_{2}=\left(\begin{array}{ccc}
1 & 0 & 0 \\
0 & 1 & 0 \\
0 & -1 & 1
\end{array}\right)\left(\begin{array}{lll}
0 & 0 & 0 \\
0 & 1 & 0 \\
0 & 0 & 1
\end{array}\right)=\left(\begin{array}{ccc}
0 & 0 & 0 \\
0 & 1 & 0 \\
0 & -1 & 1
\end{array}\right),
$$

a qual é similar a matriz $C$, com $\lambda_{1}=0$ e $\lambda_{2}=1$, caracterizando que $\left|\psi_{C}\right\rangle$ pertence à família de Jordan da matriz C. 


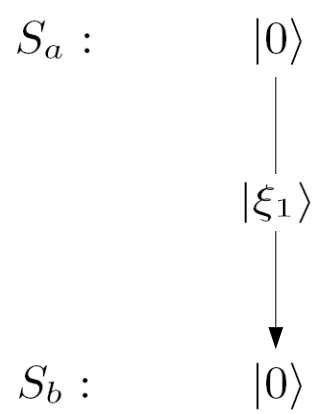

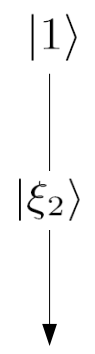

$|1\rangle$ $|2\rangle$
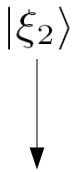

$|2\rangle$

Figura 3.4: Diagrama de correlações do estado $\left|\psi_{D}\right\rangle$. Temos que o estado $|0\rangle$ e o subespaço $\{|1\rangle,|2\rangle\}$ possuem correlações bem definidas pelos estados $\left|\xi_{1}\right\rangle$ e $\left|\xi_{2}\right\rangle$, respectivamente.

\section{Família D}

Para encontrarmos um estado $\left|\psi_{D}\right\rangle$ pertencente a esta família, vamos escolher dois estados $\left|\phi_{0}\right\rangle$ e $\left|\phi_{1}\right\rangle$ para gerar o suporte local de $\left|\psi_{D}\right\rangle$ no subsistema $S_{a b}, \mathcal{P}_{\left|\psi_{D}\right\rangle}$, tais que as respectivas matrizes sejam

$$
\Phi_{0}=\left(\begin{array}{ccc}
\lambda_{1} & 0 & 0 \\
0 & \lambda_{2} & 0 \\
0 & 0 & \lambda_{2}
\end{array}\right) \quad \text { e } \quad \Phi_{1}=\left(\begin{array}{ccc}
1 & 0 & 0 \\
0 & 1 & 0 \\
0 & 0 & 1
\end{array}\right)
$$

Dessa forma, existe um estado

$$
\left|\xi_{1}\right\rangle=\left|r_{0}\right\rangle-\lambda_{1}\left|r_{1}\right\rangle=\left(\lambda_{2}-\lambda_{1}\right)|11\rangle+|22\rangle
$$

com posto de Schmidt 2 e um estado

$$
\left|\xi_{2}\right\rangle=\left|r_{0}\right\rangle-\lambda_{2}\left|r_{1}\right\rangle=\left(\lambda_{1}-\lambda_{2}\right)|00\rangle
$$

com posto de Schmidt 1 em $\mathcal{P}_{\left|\psi_{D}\right\rangle}$. Dessa forma, podemos usar esses dois estados para decompormos $\left|\psi_{D}\right\rangle$ e, da eq. (3.8), obtemos que $\left|\psi_{D}\right\rangle$ pode ser escrito como uma superposição de três estados produto,

$$
\left|\psi_{D}\right\rangle=\frac{1}{\sqrt{3}}[|00\rangle|0\rangle+(|11\rangle+|22\rangle)|1\rangle]
$$

Note que, assim como na família B, também temos $\left|\xi_{1}\right\rangle=|00\rangle$ com um núcleo bidimensional gerado pelos estados $\left\langle 1_{a}\right|$ e $\left\langle 2_{a}\right|$. A Figura 3.4 mostra o diagrama de correlações para o estado $\left|\psi_{D}\right\rangle$ entre os subsistemas $S_{a}$ e $S_{b}$. 


\section{Família E}

Para encontramos um exemplo, $\left|\psi_{E}\right\rangle$, para esta família, também é conveniente escolhermos diretamente os estados com posto de Schmidt menor que 3 no suporte local de $\left|\psi_{E}\right\rangle$ no subsistema $S_{a b}, \mathcal{P}_{\left|\psi_{E}\right\rangle}$. Escolhemos então

$$
\left|\xi_{1}\right\rangle=|00\rangle+|11\rangle
$$

$\mathrm{e}$

$$
\left|\xi_{2}\right\rangle=|11\rangle+|22\rangle
$$

Esses dois estados são suficientes para gerar $\mathcal{P}_{\left|\psi_{E}\right\rangle}$. No entanto, como nesta família temos três auto-valores distintos, há também um terceiro estado, $\left|\xi_{3}\right\rangle$, em $\mathcal{P}_{\left|\psi_{E}\right\rangle}$ com posto de Schmidt 2. Esse estado pode ser facilmente obtido dos outros dois

$$
\left|\xi_{3}\right\rangle=\left|\xi_{1}\right\rangle-\left|\xi_{2}\right\rangle=|00\rangle-|22\rangle
$$

Também temos,

$$
\begin{aligned}
& \left|\xi_{1}\right\rangle=\left|\xi_{2}\right\rangle+\left|\xi_{3}\right\rangle \\
& \left|\xi_{2}\right\rangle=\left|\xi_{1}\right\rangle-\left|\xi_{3}\right\rangle
\end{aligned}
$$

Dessa forma, podemos escrever $\left|\psi_{E}\right\rangle$ como uma superposição de quatro estados produto de três maneiras distintas,

$$
\begin{aligned}
\left|\psi_{E}\right\rangle= & \frac{1}{2}\left[\left|\xi_{1}\right\rangle|0\rangle+\left|\xi_{2}\right\rangle|1\rangle\right] \\
& \frac{1}{2}[(|00\rangle+|11\rangle)|0\rangle+(|11\rangle+|22\rangle)|1\rangle],
\end{aligned}
$$

$\mathrm{ou}$

$$
\begin{aligned}
\left|\psi_{E}\right\rangle & =\frac{1}{2}\left[\left|\xi_{3}\right\rangle|0\rangle+\sqrt{2}\left|\xi_{2}\right\rangle|+\rangle\right] \\
& =\frac{1}{2}[(|00\rangle-|22\rangle)|0\rangle+\sqrt{2}(|11\rangle+|22\rangle|+\rangle)]
\end{aligned}
$$

ou ainda

$$
\begin{aligned}
\left|\psi_{E}\right\rangle & =\frac{1}{2}\left[\sqrt{2}\left|\xi_{1}\right\rangle|+\rangle-\left|\xi_{3}\right\rangle|1\rangle\right] \\
& =\frac{1}{2}[\sqrt{2}(|00\rangle+|11\rangle)|0\rangle-(|00\rangle-|22\rangle)|1\rangle]
\end{aligned}
$$

onde $|+\rangle=(|0\rangle+|1\rangle) / \sqrt{2}$.

A Figura 3.5 mostra o diagrama de correlações dos estados $\left|\xi_{1}\right\rangle,\left|\xi_{2}\right\rangle$ e $\left|\xi_{3}\right\rangle$. 

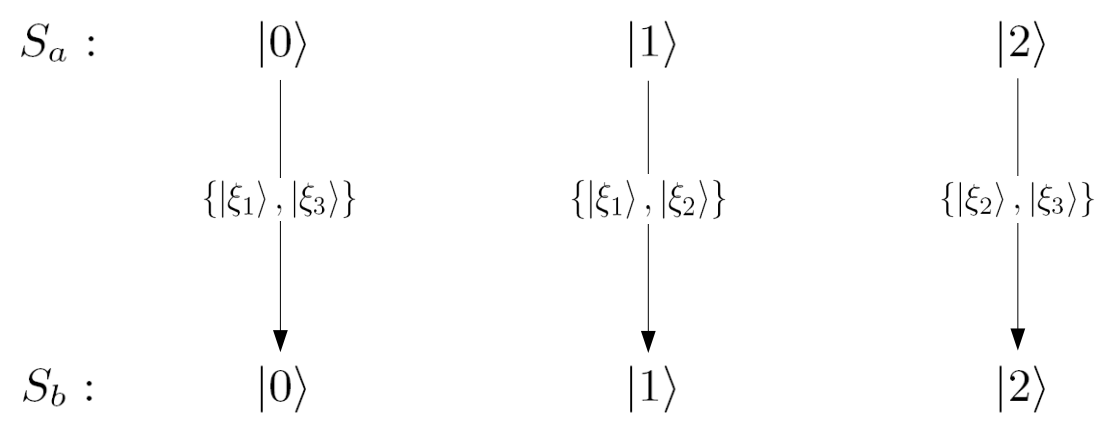

Figura 3.5: Diagrama de correlações do estado $\left|\psi_{E}\right\rangle$. Neste caso, temos os estados $|0\rangle,|1\rangle$ e $|2\rangle$ com correlações bem definidas de $S_{a}$ para $S_{b}$.

\section{Observações sobre estas famílias}

Dessa forma, encontramos cinco novas famílias para estados emaranhados de dimensionalidade $(3,3,2)$, as quais correspondem a cinco diferentes maneiras de decompormos $|\psi\rangle$ em superposições de três, quatro ou cinco estados produto. Além disso, podemos ver que existem três famílias com decomposições sub-Schmidt com quatro estados produto. Portanto, verificamos que não é apenas o posto de Schmidt que diferencia as classes de estados emaranhados. É necessário considerarmos também a natureza da decomposição e como as correlações quânticas se relacionam entre si (compare as famílias B, D e E, por exemplo). Como veremos na seção 3.3, cada uma dessas famílias corresponde a uma classe distinta de estados emaranhados com respeito a transformações do tipo SLOCC.

\subsubsection{Exemplos: dois subsistemas de quatro níveis e um qubit}

Neste caso, vamos considerar um estado emaranhado $|\psi\rangle$ de dimensionalidade $(4,4,2)$. Para estes estados, encontramos um total de treze famílias de estados emaranhados correspondentes a treze famílias de Jordan distintas.

\section{Família A}

Vamos definir a família A como os estados correspondentes à família de Jordan da matriz

$$
A=\left(\begin{array}{cccc}
\lambda_{1} & 1 & 0 & 0 \\
0 & \lambda_{1} & 1 & 0 \\
0 & 0 & \lambda_{1} & 1 \\
0 & 0 & 0 & \lambda_{1}
\end{array}\right)
$$

Para obtermos um estado $\left|\psi_{A}\right\rangle$ pertencente a esta família e sua decomposição subSchmidt, vamos escolher o suporte local de $\left|\psi_{A}\right\rangle$ no subsistema $S_{a b}, \mathcal{P}_{\left|\psi_{\mathcal{A}}\right\rangle}$, tal que este 


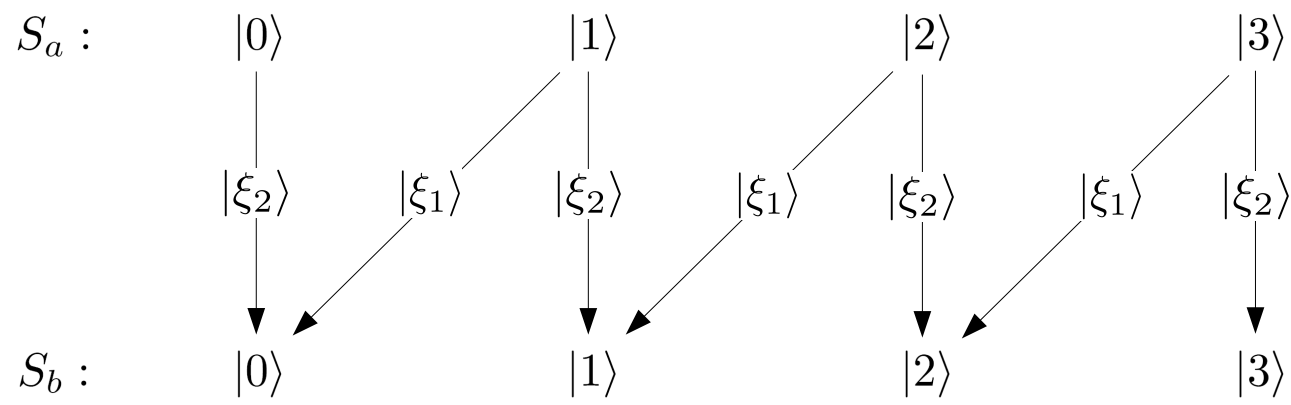

Figura 3.6: Diagrama de correlações do estado $\left|\psi_{A}\right\rangle$. Apenas o estado $|0\rangle$ possui uma correlação bem definida de $S_{a}$ para $S_{b}$ através do estado $\left|\xi_{2}\right\rangle$.

contenha os estados $\left|\phi_{0}\right\rangle$ e $\left|\phi_{1}\right\rangle$ correspondente às matrizes

$$
\Phi_{0}=\left(\begin{array}{cccc}
\lambda_{1} & 1 & 0 & 0 \\
0 & \lambda_{1} & 1 & 0 \\
0 & 0 & \lambda_{1} & 1 \\
0 & 0 & 0 & \lambda_{1}
\end{array}\right) \quad \text { e } \quad \Phi_{1}=\left(\begin{array}{cccc}
1 & 0 & 0 & 0 \\
0 & 1 & 0 & 0 \\
0 & 0 & 1 & 0 \\
0 & 0 & 0 & 1
\end{array}\right)
$$

respectivamente. Assim temos $\Phi_{1}^{-1} \Phi_{0}=A$ e o estado

$$
\left|\xi_{1}\right\rangle=\left|r_{0}\right\rangle-\lambda_{1}\left|r_{1}\right\rangle=|10\rangle+|21\rangle+|32\rangle
$$

é o único estado com posto de Schmidt menor que 4 em $\mathcal{P}_{\left|\psi_{A}\right\rangle}$ para esta família. Dessa forma, precisamos escolher um outro estado em $\mathcal{P}_{\left|\psi_{A}\right\rangle}$ como $\left|\xi_{2}\right\rangle$. Tomamos $\left|\xi_{2}\right\rangle=\left|\phi_{1}\right\rangle$. A decomposição de $\left|\psi_{A}\right\rangle$ fica

$$
\left|\psi_{A}\right\rangle=\frac{1}{\sqrt{7}}[(|10\rangle+|21\rangle+|32\rangle)|0\rangle+(|00\rangle+|11\rangle+|22\rangle+|33\rangle)|1\rangle] .
$$

Esta família pode ser considerada um análogo da família A do exemplo anterior e do estado $|W\rangle$. Particularmente, o diagrama de correlações da Figura 3.6 é bastante parecido com o da Figura 3.1.

\section{Família B}

Vamos definir a família B como os estados correspondentes à família de Jordan da matriz

$$
B=\left(\begin{array}{cccc}
\lambda_{1} & 1 & 0 & 0 \\
0 & \lambda_{1} & 0 & 0 \\
0 & 0 & \lambda_{1} & 1 \\
0 & 0 & 0 & \lambda_{1}
\end{array}\right)
$$




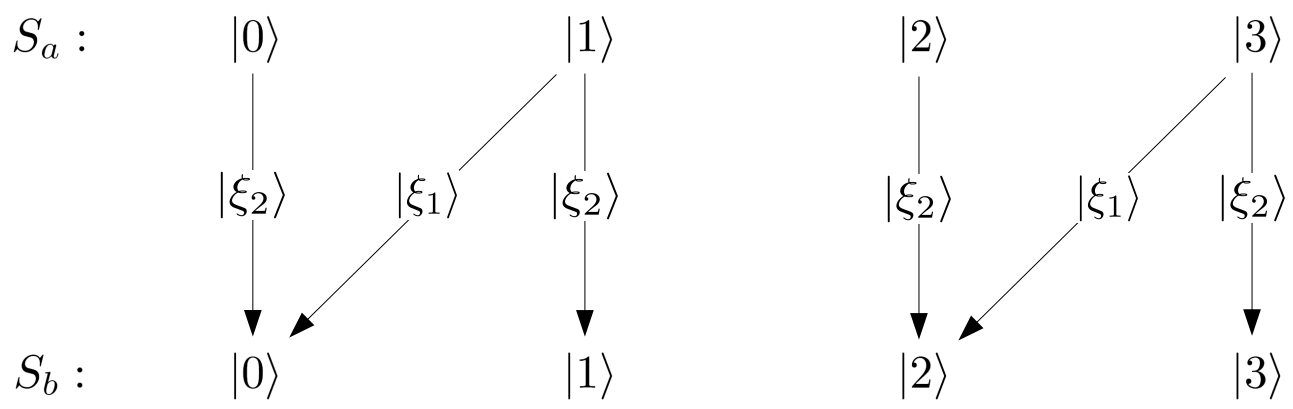

Figura 3.7: Diagrama de correlações do estado $\left|\psi_{B}\right\rangle$. O subespaço gerado por $\{|0\rangle,|2\rangle\}$ possui correlações bem definidas.

Similarmente ao exemplo anterior, para encontrarmos um exemplo $\left|\psi_{B}\right\rangle$ pertencente a esta família, vamos escolher o suporte local de $\left|\psi_{B}\right\rangle$ no subsistema $S_{a b}, \mathcal{P}_{\left|\psi_{B}\right\rangle}$, tal que este contenha os estados $\left|\phi_{0}\right\rangle$ e $\left|\phi_{1}\right\rangle$ correspondente às matrizes

$$
\Phi_{0}=\left(\begin{array}{cccc}
\lambda_{1} & 1 & 0 & 0 \\
0 & \lambda_{1} & 0 & 0 \\
0 & 0 & \lambda_{1} & 1 \\
0 & 0 & 0 & \lambda_{1}
\end{array}\right) \quad \text { e } \quad \Phi_{1}=\left(\begin{array}{cccc}
1 & 0 & 0 & 0 \\
0 & 1 & 0 & 0 \\
0 & 0 & 1 & 0 \\
0 & 0 & 0 & 1
\end{array}\right)
$$

respectivamente. Assim o estado

$$
\left|\xi_{1}\right\rangle=\left|r_{0}\right\rangle-\lambda_{1}\left|r_{1}\right\rangle=|10\rangle+|32\rangle
$$

é o único estado em $\mathcal{P}_{\left|\psi_{B}\right\rangle}$ com posto de Schmidt 2. Todos os outros estados em $\mathcal{P}_{\left|\psi_{B}\right\rangle}$ possuem posto de Schmidt 4. Assim, tomamos $\left|\xi_{2}\right\rangle=\left|\phi_{1}\right\rangle$ e podemos escrever $\left|\psi_{B}\right\rangle$ como um superposição de 6 estados produto,

$$
\left|\psi_{B}\right\rangle=\frac{1}{\sqrt{6}}[(|10\rangle+|32\rangle)|0\rangle+(|00\rangle+|11\rangle+|22\rangle+|33\rangle)|1\rangle] .
$$

A Figura 3.7 mostra o diagrama de correlações para $\left|\psi_{B}\right\rangle$.

É interessante observarmos que os estados dessa família são, na verdade, produtos tensoriais de um estado emaranhado de três qubits pertencente a classe $\mathrm{W}$ e um estado emaranhado bi-partido de dois qubits entre Alice e Bob. No caso do estado $\left|\psi_{B}\right\rangle$, por exemplo, podemos identificar o estado $\left|\psi_{B}\right\rangle$ com a situação em que Alice e Bob compartilham o estado emaranhado bi-partido de dois qubits

$$
\left|\phi^{+}\right\rangle=\frac{1}{\sqrt{2}}\left(\left|0_{a} 0_{b}\right\rangle+\left|1_{a} 1_{b}\right\rangle\right)
$$




\begin{tabular}{|c|c|c|}
\hline Alice $\left(S_{a}\right)$ & Bob $\left(S_{b}\right)$ & Carol $\left(S_{c}\right)$ \\
\hline \hline$|0\rangle \rightarrow|10\rangle$ & $|0\rangle \rightarrow|00\rangle$ & $|0\rangle \rightarrow|1\rangle$ \\
$|1\rangle \rightarrow|00\rangle$ & $|1\rangle \rightarrow|10\rangle$ & $|1\rangle \rightarrow|0\rangle$ \\
$|2\rangle \rightarrow|11\rangle$ & $|2\rangle \rightarrow|01\rangle$ & \\
$|3\rangle \rightarrow|01\rangle$ & $|3\rangle \rightarrow|11\rangle$ & \\
\hline
\end{tabular}

Tabela 3.1: Tabela da mudança de rótulos das bases dos subsistemas de Alice, Bob e Carol utilizada na identificação do estado $\left|\psi_{B}\right\rangle$ com o estado $|W\rangle \otimes\left|\phi^{+}\right\rangle$.

e também compartilham com Carol o estado emaranhado tri-partido de três qubits

$$
|W\rangle=\frac{1}{\sqrt{3}}\left(\left|0_{a} 0_{b} 1_{c}\right\rangle+\left|0_{a} 1_{b} 0_{c}\right\rangle+\left|1_{a} 0_{b} 0_{c}\right\rangle\right) .
$$

Não é difícil verificarmos que, considerando os cinco qubits, Alice, Bob e Carol de fato compartilham o estado $\left|\psi_{B}\right\rangle$. O produto tensorial resulta

$$
\begin{aligned}
& |W\rangle \otimes\left|\phi^{+}\right\rangle= \\
& \quad \frac{1}{\sqrt{6}}[(|00,00\rangle+|01,01\rangle)|1\rangle+(|00,10\rangle+|01,11\rangle+|10,00\rangle+|11,01\rangle)|0\rangle]
\end{aligned}
$$

temos que $|W\rangle \otimes|G H Z\rangle$ é idêntico a $\left|\psi_{B}\right\rangle$ a menos de uma mudança nos rótulos das bases, a qual pode ser vista na Tabela 3.1.

\section{Família C}

Vamos definir a família C como os estados pertencentes à família de Jordan da matriz

$$
C=\left(\begin{array}{cccc}
\lambda_{1} & 0 & 0 & 0 \\
0 & \lambda_{1} & 1 & 0 \\
0 & 0 & \lambda_{1} & 1 \\
0 & 0 & 0 & \lambda_{1}
\end{array}\right)
$$

Novamente, para encontramos um exemplo $\left|\psi_{C}\right\rangle$ pertencente a esta familia, vamos escolher o suporte local de $\left|\psi_{C}\right\rangle$ no subsistema $S_{a b}, \mathcal{P}_{\left|\psi_{C}\right\rangle}$, como o plano gerado pelos estados $\left|\phi_{0}\right\rangle$ e $\left|\phi_{1}\right\rangle$ correspondente às matrizes

$$
\Phi_{0}=\left(\begin{array}{cccc}
\lambda_{1} & 0 & 0 & 0 \\
0 & \lambda_{1} & 1 & 0 \\
0 & 0 & \lambda_{1} & 1 \\
0 & 0 & 0 & \lambda_{1}
\end{array}\right) \quad \text { e } \quad \Phi_{1}=\left(\begin{array}{cccc}
1 & 0 & 0 & 0 \\
0 & 1 & 0 & 0 \\
0 & 0 & 1 & 0 \\
0 & 0 & 0 & 1
\end{array}\right)
$$

respectivamente. Assim, temos $\Phi_{1}^{-1} \Phi_{0}=C$ e $\left|\psi_{C}\right\rangle$ pertence à família de Jordan de $C$. O 


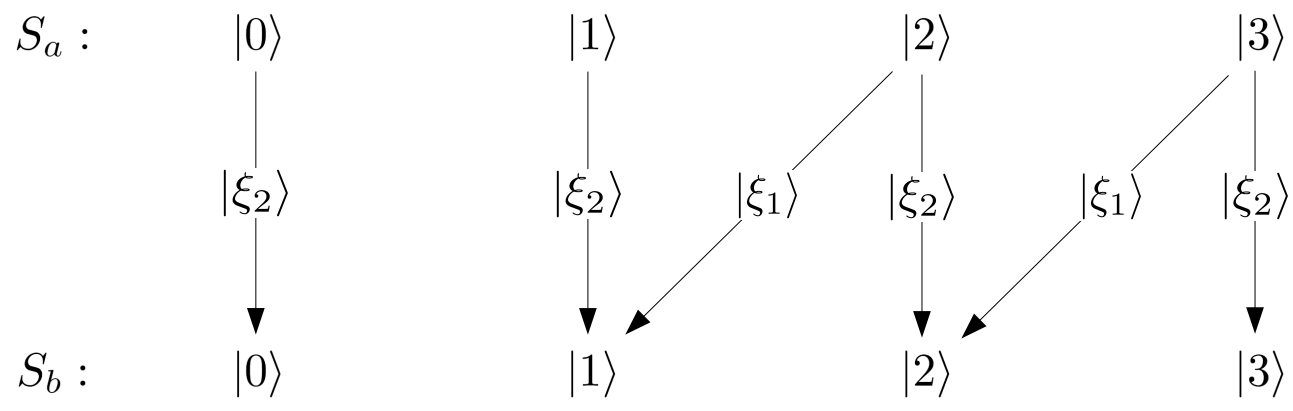

Figura 3.8: Diagrama de correlações do estado $\left|\psi_{C}\right\rangle$. Como na Figura 3.7, temos um subespaço bi-dimensional de correlações bem definidas gerado por $\{|0\rangle,|1\rangle\}$. Porém, agora temos um bloco tri-dimensional de correlações e um unidimensional, enquanto que na Figura 3.7 temos dois blocos bi-dimensionais.

estado

$$
\left|\xi_{1}\right\rangle=\left|r_{0}\right\rangle-\lambda_{1}\left|r_{1}\right\rangle=|21\rangle+|32\rangle
$$

possui posto de Schmidt 2 e é o único estado em $\mathcal{P}_{\left|\psi_{c}\right\rangle}$ com posto de Schmidt menor que 4. Tomando $\left|\xi_{2}\right\rangle=\left|\phi_{1}\right\rangle$, podemos escrever $\left|\psi_{C}\right\rangle$ como uma superposição de 6 estados produtos,

$$
\left|\psi_{C}\right\rangle=\frac{1}{\sqrt{6}}[(|21\rangle+|32\rangle)|0\rangle+(|00\rangle+|11\rangle+|22\rangle+|33\rangle)|1\rangle] .
$$

A Figura 3.8 mostra o diagrama a de correlações quânticas para o estado $\left|\psi_{C}\right\rangle$.

É interessantes compararmos os estados das famílias B e C. Os suportes locais em $C_{a}^{4} \otimes C_{b}^{4}$ dos estados pertencentes às famílias $\mathrm{B}$ e $\mathrm{C}$, isto é, os planos $\mathcal{P}_{\left|\psi_{B}\right\rangle}$ e $\mathcal{P}_{\left|\psi_{C}\right\rangle}$ obtidos dos estados $\left|\psi_{B}\right\rangle$ e $\left|\psi_{C}\right\rangle$, terão apenas um estado $\left|\xi_{1}\right\rangle$ com posto de Schmidt 2 e todos os outros estados em $\mathcal{P}_{\left|\psi_{B}\right\rangle}$ e $\mathcal{P}_{\left|\psi_{C}\right\rangle}$ terão posto de Schmidt 4. Assim qualquer estado pertencente às famílias $\mathrm{B}$ ou $\mathrm{C}$ terá uma decomposição sub-Schmidt com seis estados produto. Porém esses planos diferem em uma característica sutil. Note que no estado $\left|\psi_{B}\right\rangle$ existem dois subespaços bi-dimensionais de $C_{a}^{4}, S_{1}$ e $S_{2}$, gerados respectivamente pelas bases $\{|0\rangle,|1\rangle\}$ e $\{|2\rangle,|3\rangle\}$, nos quais podemos fazer uma medida parcial. Se o resultado da medida for $S_{1}$ ou $S_{2}$, então o estado do subsistema $S_{b}$ terá suporte local em um subespaço também bi-dimensional de $C_{b}^{4}$. Para o estado $\left|\psi_{C}\right\rangle$, esses subespaço também existem, mas nesse caso possuem dimensões 1 e 3 respectivamente. Dentro do formalismo da seção 3.1, essa diferença entre as famílias B e C aparece na dimensão dos blocos de Jordan: os postos das matrizes $\left(B-\lambda_{1}\right)^{k}$ e $\left(C-\lambda_{1}\right)^{k}$ diferem para $k=2$. 


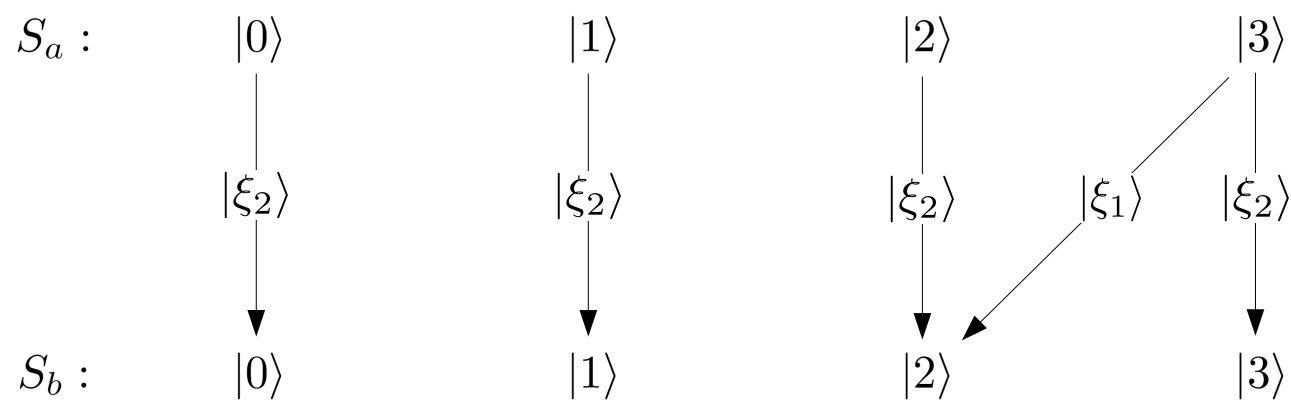

Figura 3.9: Diagrama de correlações do estado $\left|\psi_{D}\right\rangle$. Existe um subespaço tri-dimensional de correlações bem definidas gerado por $\{|0\rangle,|1\rangle,|2\rangle\}$ de $S_{a}$ para $S_{b}$.

\section{Família D}

Vamos definir a família D como os estados correspondentes a família de matrizes de Jordan do tipo da matriz

$$
D=\left(\begin{array}{cccc}
\lambda_{1} & 0 & 0 & 0 \\
0 & \lambda_{1} & 0 & 0 \\
0 & 0 & \lambda_{1} & 1 \\
0 & 0 & 0 & \lambda_{1}
\end{array}\right)
$$

Mais uma vez, para obtermos um estado desta família, $\left|\psi_{D}\right\rangle$, basta encolhermos suporte local de $\left|\psi_{D}\right\rangle$ no subsistema $S_{a b}, \mathcal{P}_{\left|\psi_{C}\right\rangle}$, tal que este possa ser gerado pelos estados $\left|\phi_{0}\right\rangle$ e $\left|\phi_{1}\right\rangle$ correspondentes as matrizes

$$
\Phi_{0}=\left(\begin{array}{cccc}
\lambda_{1} & 0 & 0 & 0 \\
0 & \lambda_{1} & 0 & 0 \\
0 & 0 & \lambda_{1} & 1 \\
0 & 0 & 0 & \lambda_{1}
\end{array}\right) \quad \text { e } \quad \Phi_{1}=\left(\begin{array}{cccc}
1 & 0 & 0 & 0 \\
0 & 1 & 0 & 0 \\
0 & 0 & 1 & 0 \\
0 & 0 & 0 & 1
\end{array}\right)
$$

respectivamente. Dessa forma, existe um estado com posto de Schmidt 1 em $\mathcal{P}_{\left|\psi_{D}\right\rangle}$, i. e., um estado produto,

$$
\left|\xi_{1}\right\rangle=\left|r_{0}\right\rangle-\lambda_{1}\left|r_{1}\right\rangle=|32\rangle .
$$

Como não existe outro estado com posto de Schmidt menor que 4 em $\mathcal{P}_{D}$, tomamos $\left|\xi_{2}\right\rangle=\left|\phi_{1}\right\rangle$. Dessa forma, podemos escrever $\left|\psi_{D}\right\rangle$ como uma superposição de cinco estados produto,

$$
\left|\psi_{D}\right\rangle=\frac{1}{\sqrt{5}}[|32\rangle|0\rangle+(|00\rangle+|11\rangle+|22\rangle+|33\rangle)|1\rangle]
$$

O diagrama de correlações do estado $\left|\psi_{D}\right\rangle$ pode ser visto na figura 3.2.3. 


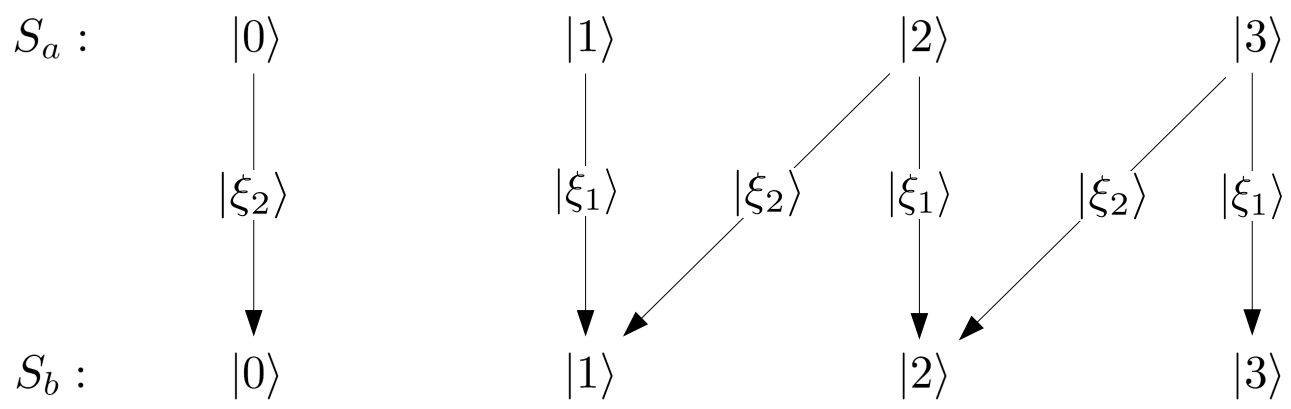

Figura 3.10: Diagrama de correlações do estado $\left|\psi_{E}\right\rangle$. Os estados $|0\rangle$ e $|1\rangle$ possuem correlações bem definidas de $S_{a}$ para $S_{b}$.

\section{Família E}

Com a família E, começamos com os exemplos das famílias com dois autovalores distintos. Vamos defini-la como os estados associados à família de Jordan da matriz

$$
E=\left(\begin{array}{cccc}
\lambda_{1} & 0 & 0 & 0 \\
0 & \lambda_{2} & 1 & 0 \\
0 & 0 & \lambda_{2} & 1 \\
0 & 0 & 0 & \lambda_{2}
\end{array}\right)
$$

onde $\lambda_{1} \neq \lambda_{2}$.

Para qualquer estado $\left|\psi_{E}\right\rangle$ nesta família, temos dois autovalores distintos correspondentes a dois estados com posto de Schmidt 3 no suporte local do subsistema $S_{a b}, \mathcal{P}_{\left|\psi_{E}\right\rangle}$. Para encontramos um exemplo de um estado $\left|\psi_{E}\right\rangle$, é conveniente escolhermos os estados $\left|\xi_{1}\right\rangle$ e $\left|\xi_{2}\right\rangle$ em $\mathcal{P}_{\left|\psi_{E}\right\rangle}$ com posto de Schmidt 3. Escolhemos

$$
\left|\xi_{1}\right\rangle=|11\rangle+|22\rangle+|33\rangle
$$

e

$$
\left|\xi_{2}\right\rangle=|00\rangle+|21\rangle+|32\rangle \text {. }
$$

Dessa forma, escrevemos $\left|\psi_{E}\right\rangle$ com uma superposição de 6 estados produto,

$$
\left|\psi_{E}\right\rangle=\frac{1}{\sqrt{6}}[(|11\rangle+|22\rangle+|33\rangle)|0\rangle+(|00\rangle+|21\rangle+|32\rangle)|1\rangle]
$$

A Figura 3.10 mostra o diagrama de correlações do estado $\left|\psi_{E}\right\rangle$.

Para mostrarmos que $\left|\psi_{E}\right\rangle$ pertence de fato a esta família, precisamos de um estado com posto de Schmidt 4 em $\mathcal{P}_{\left|\psi_{E}\right\rangle}$. Os estados $\left|\xi_{1}\right\rangle$ e $\left|\xi_{2}\right\rangle$ correspondem respectivamente 
às seguintes matrizes

$$
\Xi_{1}=\left(\begin{array}{cccc}
0 & 0 & 0 & 0 \\
0 & 1 & 0 & 0 \\
0 & 0 & 1 & 0 \\
0 & 0 & 0 & 1
\end{array}\right) \quad \text { e } \quad \Xi_{2}=\left(\begin{array}{cccc}
1 & 0 & 0 & 0 \\
0 & 0 & 1 & 0 \\
0 & 0 & 0 & 1 \\
0 & 0 & 0 & 0
\end{array}\right)
$$

Assim podemos definir a matriz

$$
\Phi_{1}=\Xi_{1}+\Xi_{2}=\left(\begin{array}{cccc}
1 & 0 & 0 & 0 \\
0 & 1 & 1 & 0 \\
0 & 0 & 1 & 1 \\
0 & 0 & 0 & 1
\end{array}\right)
$$

que é inversível e corresponde a um estado $\left|\phi_{1}\right\rangle$ com posto de Schmidt 4 em $\mathcal{P}_{\left|\psi_{E}\right\rangle}$. Temos também que

$$
\Phi_{1}^{-1} \Xi_{1}=\left(\begin{array}{cccc}
1 & 0 & 0 & 0 \\
0 & 1 & -1 & 1 \\
0 & 0 & 1 & -1 \\
0 & 0 & 0 & 1
\end{array}\right)\left(\begin{array}{cccc}
0 & 0 & 0 & 0 \\
0 & 1 & 0 & 0 \\
0 & 0 & 1 & 0 \\
0 & 0 & 0 & 1
\end{array}\right)=\left(\begin{array}{cccc}
0 & 0 & 0 & 0 \\
0 & 1 & -1 & 1 \\
0 & 0 & 1 & -1 \\
0 & 0 & 0 & 1
\end{array}\right)
$$

que é similar à matriz $E \operatorname{com} \lambda_{1}=0$ e $\lambda_{2}=1$ e portanto $\left|\psi_{E}\right\rangle$ pertence à família de Jordan da matriz $E$.

\section{Família F}

Vamos definir a família F como os estados correspondentes à família de Jordan da matriz

$$
F=\left(\begin{array}{cccc}
\lambda_{1} & 1 & 0 & 0 \\
0 & \lambda_{1} & 0 & 0 \\
0 & 0 & \lambda_{2} & 1 \\
0 & 0 & 0 & \lambda_{2}
\end{array}\right)
$$

onde $\lambda_{1} \neq \lambda_{2}$.

Novamente, a melhor maneira de construirmos um estado $\left|\psi_{F}\right\rangle$ pertencente a esta família é escolhendo os estados com posto de Schmidt menor que 4 no suporte local de $\left|\psi_{F}\right\rangle$ no subsistema $S_{a b}, \mathcal{P}_{\left|\psi_{F}\right\rangle}$. Pela matriz da família de Jordan $F$, sabemos que existem dois estados com posto de Schmidt $3 \mathrm{em} \mathcal{P}_{\left|\psi_{F}\right\rangle}$. Então, escolhemos

$$
\left|\xi_{1}\right\rangle=|10\rangle+|22\rangle+|33\rangle
$$




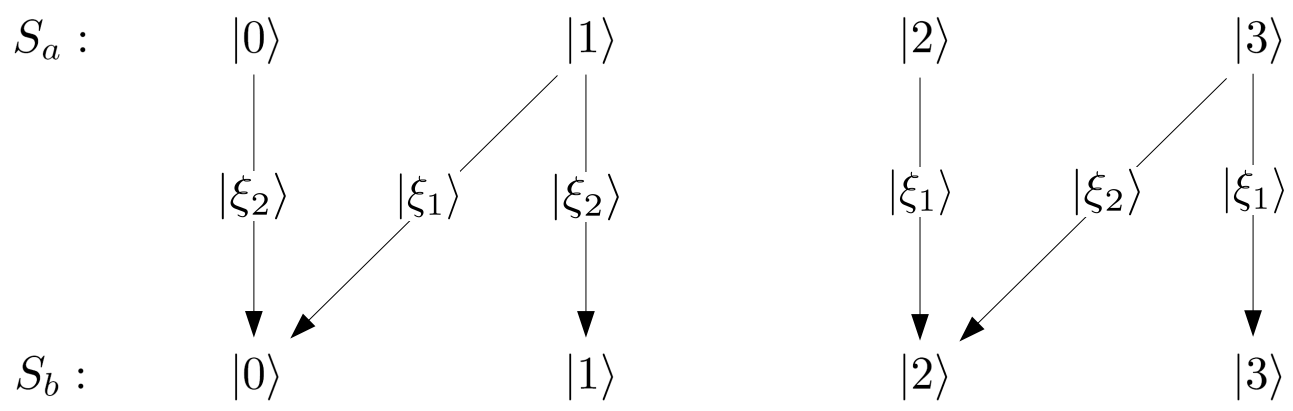

Figura 3.11: Diagrama de correlações do estado $\left|\psi_{F}\right\rangle$. Os estados $|0\rangle$ e $|2\rangle$ possuem correlações bem definidas de $S_{a}$ para $S_{b}$. Comparando com a Figura 3.10, também verificamos a diferença na dimensão dos blocos de correlações como entre as famílias B e C.

e

$$
\left|\xi_{2}\right\rangle=|00\rangle+|11\rangle+|32\rangle
$$

Assim, podemos escrever o estado tri-partido $\left|\psi_{F}\right\rangle$ como

$$
\left|\psi_{F}\right\rangle=\frac{1}{\sqrt{6}}[(|10\rangle+|22\rangle+|33\rangle)|0\rangle+(|00\rangle+|11\rangle+|32\rangle)|1\rangle] .
$$

O diagrama de correlações pode ser visto na figura 3.11 .

É interessante compararmos também as famílias E e F. Os postos das matrizes $\left(E-\lambda_{1}\right)^{k}$ e $\left(F-\lambda_{1}\right)^{k}$ são diferentes para $k \geq 2$, enquanto que os postos das matrizes $\left(E-\lambda_{2}\right)^{k}$ e $\left(F-\lambda_{2}\right)^{k}$ são diferentes para $k \geq 3$. Assim, tanto os estados pertencentes à família E quanto os pertencentes à família $\mathrm{F}$ resultam em planos, $P_{\left|\psi_{E}\right\rangle}$ e $\mathcal{P}_{\left|\psi_{F}\right\rangle}$ pertencentes a $C_{a}^{4} \otimes C_{b}^{4}$, com dois estados com posto de Schmidt 3. As decomposições sub-Schmidt dos estados dessas famílias envolvem seis estados produto e são bastante parecidas. No entanto, observando os diagramas de correlações, vemos que, para os estados da família E, existe um bloco de correlações relacionando subespaços tri-dimensional de $S_{a}$ e $S_{b}$ e um bloco relacionado subespaços unidimensional enquanto que, para os estados da família $\mathrm{F}$, existem dois blocos relacionando subespaços bi-dimensionais.

Por fim, vamos mostrar que $\left|\psi_{F}\right\rangle$ pertence a família F. As matrizes correspondentes aos estados $\left|\xi_{1}\right\rangle$ e $\left|\xi_{2}\right\rangle$ são

$$
\Xi_{1}=\left(\begin{array}{cccc}
0 & 1 & 0 & 0 \\
0 & 0 & 0 & 0 \\
0 & 0 & 1 & 0 \\
0 & 0 & 0 & 1
\end{array}\right) \quad \text { e } \quad \Xi_{2}=\left(\begin{array}{cccc}
1 & 0 & 0 & 0 \\
0 & 1 & 0 & 0 \\
0 & 0 & 0 & 1 \\
0 & 0 & 0 & 0
\end{array}\right)
$$

respectivamente. Essas matrizes possuem posto 3. Para obtermos uma matriz de posto 4 
em $\mathcal{P}_{\left|\psi_{F}\right\rangle}$, definimos

$$
\Phi_{1}=\Xi_{1}+\Xi_{2}=\left(\begin{array}{cccc}
1 & 1 & 0 & 0 \\
0 & 1 & 0 & 0 \\
0 & 0 & 1 & 1 \\
0 & 0 & 0 & 1
\end{array}\right)
$$

Assim temos

$$
\Phi_{1}^{-1} \Xi_{1}=\left(\begin{array}{cccc}
1 & -1 & 0 & 0 \\
0 & 1 & 0 & 0 \\
0 & 0 & 1 & -1 \\
0 & 0 & 0 & 1
\end{array}\right)\left(\begin{array}{cccc}
0 & 1 & 0 & 0 \\
0 & 0 & 0 & 0 \\
0 & 0 & 1 & 0 \\
0 & 0 & 0 & 1
\end{array}\right)=\left(\begin{array}{cccc}
0 & 1 & 0 & 0 \\
0 & 0 & 0 & 0 \\
0 & 0 & 1 & -1 \\
0 & 0 & 0 & 1
\end{array}\right)
$$

que é similar a $F \operatorname{com} \lambda_{1}=0$ e $\lambda_{2}=1$ e portanto $\left|\psi_{F}\right\rangle$ pertence à família de Jordan da matriz $F$.

\section{Família G}

Vamos definir a família G como a família de Jordan caracterizada pela matriz

$$
G=\left(\begin{array}{cccc}
\lambda_{1} & 0 & 0 & 0 \\
0 & \lambda_{2} & 0 & 0 \\
0 & 0 & \lambda_{2} & 1 \\
0 & 0 & 0 & \lambda_{2}
\end{array}\right)
$$

onde $\lambda_{1} \neq \lambda_{2}$.

Mais uma vez, para obtermos um exemplo de um estado, $\left|\psi_{G}\right\rangle$, pertencente a esta família, é conveniente escolhermos os estados no suporte local de $\left|\psi_{G}\right\rangle$ no subsistema $S_{a b}$, $\mathcal{P}_{\left|\psi_{G}\right\rangle}$, com posto de Schmidt menor que 4. Da matriz G, sabemos que existem um estado com posto de Schmidt 3 e outro com posto de Schmidt 2. Escolhemos

$$
\left|\xi_{1}\right\rangle=|11\rangle+|22\rangle+|33\rangle
$$

e

$$
\left|\xi_{2}\right\rangle=|00\rangle+|23\rangle
$$

Dessa forma, $\left|\psi_{G}\right\rangle$ pode ser decomposto em 5 estados produto,

$$
\left|\psi_{G}\right\rangle=\frac{1}{\sqrt{5}}[(|11\rangle+|22\rangle+|33\rangle)|0\rangle+(|00\rangle+|23\rangle)|1\rangle] .
$$

O diagrama de correlações para o estado $\left|\psi_{G}\right\rangle$ pode ser visto na figura 3.12.

Para mostrarmos que $\left|\psi_{G}\right\rangle$ pertence à família de Jordan correspondente a matriz $G$, 


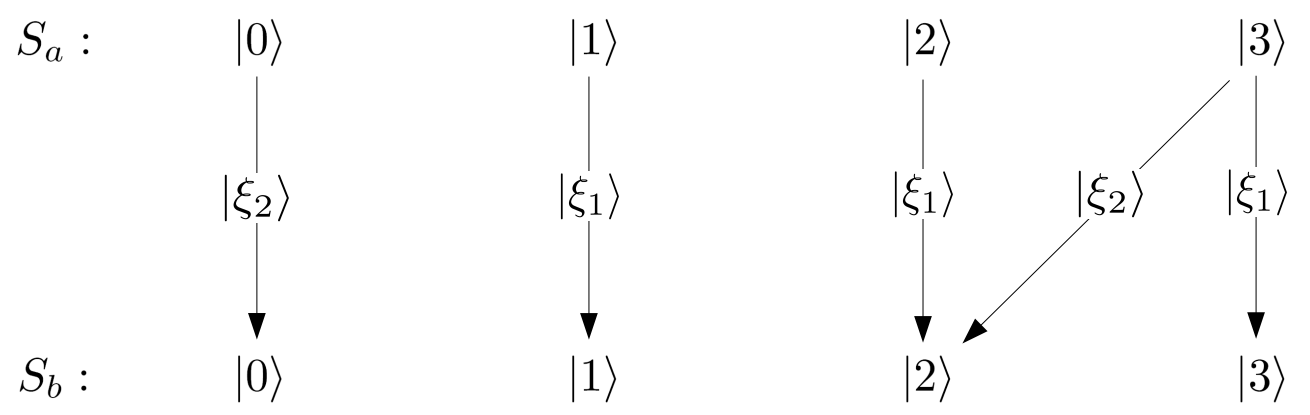

Figura 3.12: Diagrama de correlações do estado $\left|\psi_{G}\right\rangle$. O estado $|0\rangle$ e o subespaço gerado por $\{|1\rangle,|2\rangle\}$ possuem correlações bem definidas $S_{a}$ para $S_{b}$.

precisamos de um estado com posto de Schmidt 4 em $\mathcal{P}_{\left|\psi_{G}\right\rangle}$. As matriz correspondentes aos estados $\left|\xi_{1}\right\rangle$ e $\left|\xi_{2}\right\rangle$ são

$$
\Xi_{1}=\left(\begin{array}{cccc}
0 & 0 & 0 & 0 \\
0 & 1 & 0 & 0 \\
0 & 0 & 1 & 0 \\
0 & 0 & 0 & 1
\end{array}\right) \quad \text { e } \quad \Xi_{2}=\left(\begin{array}{cccc}
1 & 0 & 0 & 0 \\
0 & 0 & 0 & 0 \\
0 & 0 & 0 & 1 \\
0 & 0 & 0 & 0
\end{array}\right)
$$

respectivamente. Assim podemos definir a matriz

$$
\Phi_{1}=\Xi_{1}+\Xi_{2}=\left(\begin{array}{cccc}
1 & 0 & 0 & 0 \\
0 & 1 & 0 & 0 \\
0 & 0 & 1 & 1 \\
0 & 0 & 0 & 1
\end{array}\right)
$$

que corresponde a um estado com posto de Schmidt 4. Dessa forma, temos

$$
\Phi_{1}^{-1} \Xi_{1}=\left(\begin{array}{cccc}
1 & 0 & 0 & 0 \\
0 & 1 & 0 & 0 \\
0 & 0 & 1 & 0 \\
0 & 0 & -1 & 1
\end{array}\right)\left(\begin{array}{cccc}
0 & 0 & 0 & 0 \\
0 & 1 & 0 & 0 \\
0 & 0 & 1 & 0 \\
0 & 0 & 0 & 1
\end{array}\right)=\left(\begin{array}{cccc}
0 & 0 & 0 & 0 \\
0 & 1 & 0 & 0 \\
0 & 0 & 1 & 0 \\
0 & 0 & -1 & 1
\end{array}\right)
$$

que é similar a $G \operatorname{com} \lambda_{1}=0$ e $\lambda_{2}=1$ e portanto $\left|\psi_{G}\right\rangle$ pertence à família de Jordan da matriz $G$. 

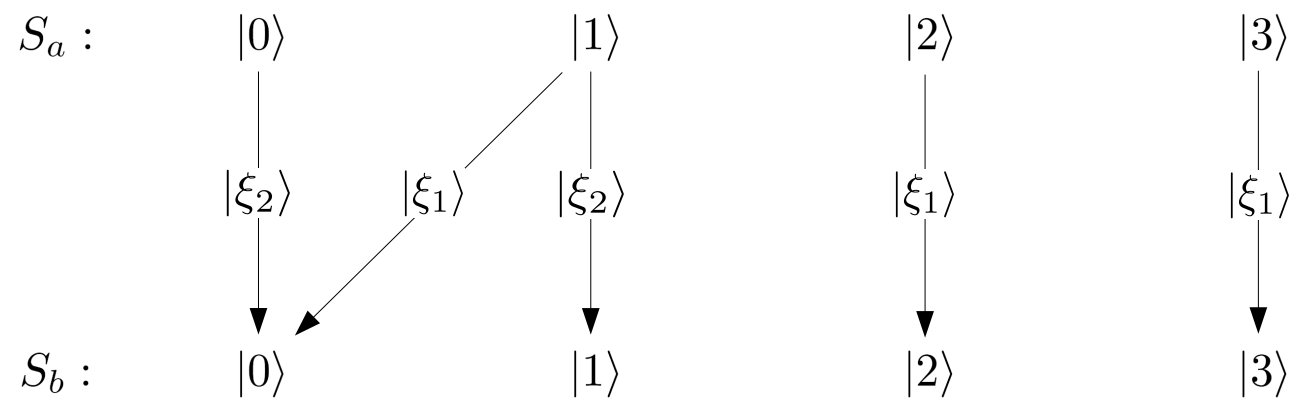

$|1\rangle$

$|2\rangle$

$|3\rangle$

Figura 3.13: Diagrama de correlações do estado $\left|\psi_{H}\right\rangle$. O estado $|0\rangle$ e o subespaço gerado por $\{|2\rangle,|3\rangle\}$ possuem correlações bem definidas de $S_{a}$ para $S_{b}$.

\section{Família H}

Vamos definir a família $\mathrm{H}$ como a família dos estados caracterizados pela matriz

$$
H=\left(\begin{array}{cccc}
\lambda_{1} & 1 & 0 & 0 \\
0 & \lambda_{1} & 0 & 0 \\
0 & 0 & \lambda_{2} & 0 \\
0 & 0 & 0 & \lambda_{2}
\end{array}\right)
$$

onde $\lambda_{1} \neq \lambda_{2}$.

Para encontrarmos um estado $\left|\psi_{H}\right\rangle$ pertencente esta família, é conveniente mais uma vez escolhermos os estado no suporte local de $\left|\psi_{H}\right\rangle, \mathcal{P}_{\left|\psi_{H}\right\rangle}$, com posto de Schmidt menor que 4. A partir da matriz $H$, temos que $\mathcal{P}_{\left|\psi_{H}\right\rangle}$ contém um estado com posto de Schmidt 2 e outro com posto 3. Escolhemos

$$
\left|\xi_{1}\right\rangle=|21\rangle+|22\rangle+|33\rangle
$$

e

$$
\left|\xi_{2}\right\rangle=|00\rangle+|11\rangle
$$

Dessa forma, $\left|\psi_{H}\right\rangle$ pode ser decomposto em 5 estados produto,

$$
\left|\psi_{H}\right\rangle=\frac{1}{\sqrt{5}}[(|22\rangle+|33\rangle+|10\rangle)|0\rangle+(|00\rangle+|11\rangle)|1\rangle] .
$$

O diagrama de correlações do estado $\left|\psi_{H}\right\rangle$ pode ser visto na figura 3.13 , o qual é ligeiramente diferente do diagrama de correlações 3.12 do estado $\left|\psi_{G}\right\rangle$ da família anterior . É importante salientarmos que, para diferenciarmos os estados das famílias $\mathrm{G}$ e H, também precisamos verificar os postos das matrizes $(F-\lambda)^{k}$ e $(G-\lambda)^{k}$ para $k \geq 2$ tanto para $\lambda=\lambda_{1}$ quanto $\lambda=\lambda_{2}$.

Para mostrarmos que $\left|\psi_{H}\right\rangle$ pertence à família $\mathrm{H}$, precisamos de um estado com posto 
de Schmidt $4 \mathrm{em} \mathcal{P}_{H}$. Esse estado pode ser obtido das matrizes correspondente aos estados $\left|\xi_{1}\right\rangle$ e $\left|\xi_{2}\right\rangle$

$$
\Xi_{1}=\left(\begin{array}{cccc}
0 & 1 & 0 & 0 \\
0 & 0 & 0 & 0 \\
0 & 0 & 1 & 0 \\
0 & 0 & 0 & 1
\end{array}\right) \quad \text { e } \quad \Xi_{2}=\left(\begin{array}{cccc}
1 & 0 & 0 & 0 \\
0 & 1 & 0 & 0 \\
0 & 0 & 0 & 0 \\
0 & 0 & 0 & 0
\end{array}\right)
$$

respectivamente. Definindo o estado $\left|\phi_{1}\right\rangle=\left|\xi_{1}\right\rangle+\left|\xi_{2}\right\rangle$, correspondente a matriz

$$
\Phi_{1}=\Xi_{1}+\Xi_{2}=\left(\begin{array}{cccc}
1 & 1 & 0 & 0 \\
0 & 1 & 0 & 0 \\
0 & 0 & 1 & 0 \\
0 & 0 & 0 & 1
\end{array}\right)
$$

obtemos

$$
\Phi_{1}^{-1} \Xi_{2}\left(\begin{array}{cccc}
1 & 0 & 0 & 0 \\
-1 & 1 & 0 & 0 \\
0 & 0 & 1 & 0 \\
0 & 0 & 0 & 1
\end{array}\right)\left(\begin{array}{cccc}
1 & 0 & 0 & 0 \\
0 & 1 & 0 & 0 \\
0 & 0 & 0 & 0 \\
0 & 0 & 0 & 0
\end{array}\right)=\left(\begin{array}{cccc}
1 & 0 & 0 & 0 \\
-1 & 1 & 0 & 0 \\
0 & 0 & 0 & 0 \\
0 & 0 & 0 & 0
\end{array}\right)
$$

que é similar a $H \operatorname{com} \lambda_{1}=1$ e $\lambda_{2}=0$ e portanto $\left|\psi_{H}\right\rangle$ pertence à família de Jordan da matriz $H$.

\section{Família I}

Vamos definir a família I como a família de estados correspondentes à matriz

$$
I=\left(\begin{array}{cccc}
\lambda_{1} & 0 & 0 & 0 \\
0 & \lambda_{2} & 0 & 0 \\
0 & 0 & \lambda_{2} & 0 \\
0 & 0 & 0 & \lambda_{2}
\end{array}\right)
$$

onde $\lambda_{1} \neq \lambda_{2}$.

Para construirmos um estado pertencente a esta família, $\left|\psi_{I}\right\rangle$, basta escolhermos o suporte local de $\left|\psi_{I}\right\rangle, \mathcal{P}_{\left|\psi_{I}\right\rangle}$, tal que este contenha os estados $\left|\phi_{0}\right\rangle$ e $\left|\phi_{1}\right\rangle$ correspondentes às matrizes

$$
\Phi_{0}=\left(\begin{array}{cccc}
\lambda_{1} & 0 & 0 & 0 \\
0 & \lambda_{2} & 0 & 0 \\
0 & 0 & \lambda_{2} & 0 \\
0 & 0 & 0 & \lambda_{2}
\end{array}\right) \quad \text { e } \quad \Phi_{1}=\left(\begin{array}{cccc}
1 & 0 & 0 & 0 \\
0 & 1 & 0 & 0 \\
0 & 0 & 1 & 0 \\
0 & 0 & 0 & 1
\end{array}\right)
$$




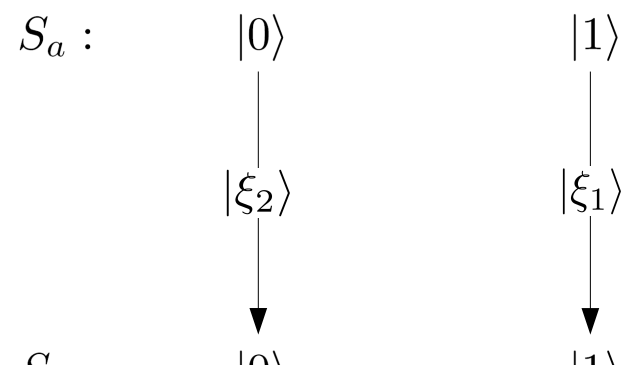

$1\rangle \quad|2\rangle$

$|3\rangle$

$S_{b}: \quad|0\rangle$

$|1\rangle$
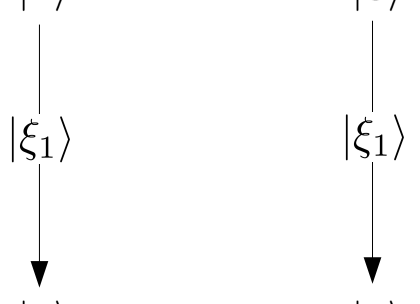

$|3\rangle$

Figura 3.14: Diagrama de correlações do estado $\left|\psi_{I}\right\rangle$. O estado $|0\rangle$ e o subespaço gerado por $\{|1\rangle,|2\rangle,|3\rangle\}$ possuem correlações bem definidas de $S_{a}$ para $S_{b}$.

respectivamente. Assim, temos que $\Phi_{1}^{-1} \Phi_{0}=I$ e existem dois estados com posto de Schmidt menor que 4: o estado

$$
\left|\xi_{1}\right\rangle=\left|\phi_{0}\right\rangle-\lambda_{1}\left|\phi_{1}\right\rangle=\left(\lambda_{2}-\lambda_{1}\right)(|11\rangle+|22\rangle+|33\rangle)
$$

com posto de Schmidt 3; e o estado

$$
\left|\xi_{2}\right\rangle=\left|\xi_{0}\right\rangle-\lambda_{2}\left|\xi_{1}\right\rangle=\left(\lambda_{1}-\lambda_{2}\right)|00\rangle
$$

que corresponde a um estado produto com posto de Schmidt 1. Dessa forma, temos que o estado emaranhado tripartido $\left|\psi_{I}\right\rangle$ pode ser decomposto em 4 estados produto,

$$
\left|\psi_{I}\right\rangle=\frac{1}{2}[(|11\rangle+|22\rangle+|33\rangle)|0\rangle+|00\rangle|1\rangle] .
$$

O diagrama de correlações do estado $\left|\psi_{I}\right\rangle$ pode ser visto na Figura 3.14.

\section{Família J}

Vamos definir a família $\mathbf{J}$ como os estados correspondentes à família Jordan da matriz

$$
J=\left(\begin{array}{cccc}
\lambda_{1} & 0 & 0 & 0 \\
0 & \lambda_{1} & 0 & 0 \\
0 & 0 & \lambda_{2} & 0 \\
0 & 0 & 0 & \lambda_{2}
\end{array}\right)
$$

onde $\lambda_{1} \neq \lambda_{2}$.

Para obtermos um estado $\left|\psi_{J}\right\rangle$, pertencente a esta família, vamos escolher o suporte local de $\left|\psi_{J}\right\rangle$ no subsistema $S_{a b}, \mathcal{P}_{\left|\psi_{J}\right\rangle}$, tal que este contenha os estados $\left|\phi_{0}\right\rangle$ e $\left|\phi_{1}\right\rangle$ corres- 


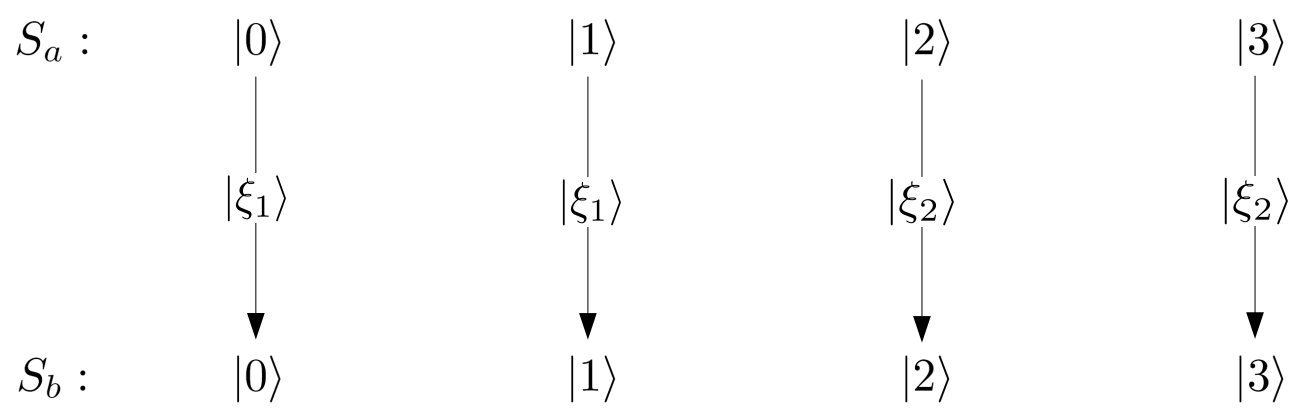

Figura 3.15: Diagrama de correlações do estado $\left|\psi_{J}\right\rangle$. Os subespaços gerados por $\{|0\rangle,|1\rangle\}$ e $\{|2\rangle,|3\rangle\}$ possuem correlações bem definidas de $S_{a}$ para $S_{b}$.

pondentes as matrizes

$$
\Phi_{0}=\left(\begin{array}{cccc}
\lambda_{1} & 0 & 0 & 0 \\
0 & \lambda_{1} & 0 & 0 \\
0 & 0 & \lambda_{2} & 0 \\
0 & 0 & 0 & \lambda_{2}
\end{array}\right) \quad \text { e } \quad \Phi_{1}=\left(\begin{array}{cccc}
1 & 0 & 0 & 0 \\
0 & 1 & 0 & 0 \\
0 & 0 & 1 & 0 \\
0 & 0 & 0 & 1
\end{array}\right)
$$

respectivamente. Dessa forma, $\mathcal{P}_{\left|\psi_{J}\right\rangle}$ contém dois estados com postos Schmidt 2,

$$
\begin{aligned}
& \left|\xi_{1}\right\rangle=\left|r_{0}\right\rangle-\lambda_{1}\left|r_{1}\right\rangle=\left(\lambda_{2}-\lambda_{1}\right)(|22\rangle+|33\rangle) \\
& \left|\xi_{2}\right\rangle=\left|r_{0}\right\rangle-\lambda_{2}\left|r_{1}\right\rangle=\left(\lambda_{1}-\lambda_{2}\right)(|00\rangle+|11\rangle),
\end{aligned}
$$

e todos os outros estados em $\mathcal{P}_{\left|\psi_{J}\right\rangle}$ possuem posto de Schmidt 4. Usando $\left|\xi_{1}\right\rangle$ e $\left|\xi_{2}\right\rangle$, podemos escrever $\left|\psi_{J}\right\rangle$ como um superposição de 4 estados produto

$$
\left|\psi_{J}\right\rangle=\frac{1}{2}[(|00\rangle+|11\rangle)|0\rangle+(|22\rangle+|33\rangle)|1\rangle] .
$$

O diagrama de correlações pode ser visto na Figura 3.15.

É interessante analisamos os estados desta família com um pouco mais de cuidado. Assim como a família B, estados desta família são produtos tensoriais de dois estados emaranhados: Um estado tri-partido da família GHZ, compartilhado entre Alice, Bob e Carol e um estado bi-partido compartilhado entre Alice e Bob. Particularmente, podemos verificar que $\left|\psi_{J}\right\rangle$ corresponde ao produto tensorial do estado emaranhado tripartido

$$
|G H Z\rangle=\frac{1}{\sqrt{2}}(|000\rangle+|111\rangle)
$$

com o estado de Bell

$$
\left|\phi^{+}\right\rangle=\frac{1}{\sqrt{2}}(|00\rangle+|11\rangle)
$$


Explicitamente, temos

$$
|G H Z\rangle \otimes\left|\phi^{+}\right\rangle=\frac{1}{2}[(|00,00\rangle+|01,01\rangle)|0\rangle+(|10,10\rangle+|11,11\rangle)|1\rangle] .
$$

Comparando (3.10) e (3.11) é fácil vermos que $\left|\psi_{J}\right\rangle$ e $|G H Z\rangle \otimes\left|\phi^{+}\right\rangle$diferem apenas pela rotulação das bases nos subsistemas de Alice e Bob.

\section{Família K}

Vamos definir a família K como os estados correspondentes a família de Jordan da matriz

$$
K=\left(\begin{array}{cccc}
\lambda_{1} & 0 & 0 & 0 \\
0 & \lambda_{2} & 0 & 0 \\
0 & 0 & \lambda_{3} & 1 \\
0 & 0 & 0 & \lambda_{3}
\end{array}\right)
$$

onde $\lambda_{1} \neq \lambda_{2} \neq \lambda_{3}$.

Para encontramos um exemplo $\left|\psi_{K}\right\rangle$ para essa família, é conveniente escolhermos os estados no suporte local de $\left|\psi_{K}\right\rangle$ no subsistema $S_{a b}, \mathcal{P}_{\left|\psi_{K}\right\rangle}$, com posto de Schmidt menor que $n$. Da matriz $K$, podemos ver que existem três estados com posto de Schmidt $3 \mathrm{em}$ $\mathcal{P}_{\left|\psi_{K}\right\rangle}$. Primeiro, vamos escolher os estados $\left|\xi_{3}\right\rangle$ e $\left|\xi_{2}\right\rangle$, correspondentes aos autovalores $\lambda_{3}$ e $\lambda_{2}$,

$$
\begin{aligned}
& \left|\xi_{3}\right\rangle=\frac{1}{\sqrt{6}}(|00\rangle+2|11\rangle+|32\rangle) \\
& \left|\xi_{2}\right\rangle=\frac{1}{\sqrt{5}}(|00\rangle-\sqrt{2}(|22\rangle+|33\rangle)) .
\end{aligned}
$$

O estado $\left|\xi_{1}\right\rangle$, correspondente ao autovalor $\lambda_{1}$, é uma combinação linear de $\left|\xi_{2}\right\rangle$ e $\left|\xi_{3}\right\rangle$,

$$
\left|\xi_{1}\right\rangle=\frac{\sqrt{6}}{3}\left(\left|\xi_{3}\right\rangle-\sqrt{\frac{5}{6}}\left|\xi_{2}\right\rangle\right)=\frac{1}{3}[2|11\rangle+|32\rangle+\sqrt{2}(|22\rangle+|33\rangle)],
$$

e temos também que

$$
\begin{aligned}
& \left|\xi_{2}\right\rangle=\frac{3}{\sqrt{5}}\left(\left|\xi_{1}\right\rangle-\frac{\sqrt{6}}{3}\left|\xi_{3}\right\rangle\right) \\
& \left|\xi_{3}\right\rangle=\frac{3}{\sqrt{6}}\left(\left|\xi_{1}\right\rangle+\frac{\sqrt{5}}{3}\left|\xi_{2}\right\rangle\right) .
\end{aligned}
$$


A decomposição de Schmidt do estado $\left|\xi_{1}\right\rangle$ fica

$$
\left|\xi_{1}\right\rangle=\frac{1}{3}\left(2|11\rangle+2\left|a_{1}\right\rangle\left|b_{1}\right\rangle+\left|a_{2}\right\rangle\left|b_{2}\right\rangle\right)
$$

onde

$$
\begin{array}{ll}
\left|a_{1}\right\rangle=-\sqrt{\frac{2}{3}}\left|2_{a}\right\rangle-\frac{1}{\sqrt{3}}\left|3_{a}\right\rangle & \left|b_{1}\right\rangle=-\frac{1}{\sqrt{3}}\left|2_{b}\right\rangle-\sqrt{\frac{2}{3}}\left|3_{b}\right\rangle \\
\left|a_{2}\right\rangle=-\frac{1}{\sqrt{3}}\left|2_{a}\right\rangle+\sqrt{\frac{2}{3}}\left|3_{a}\right\rangle & \left|b_{2}\right\rangle=-\sqrt{\frac{2}{3}}\left|2_{b}\right\rangle+\frac{1}{\sqrt{3}}\left|3_{b}\right\rangle .
\end{array}
$$

Dessa forma, $\left|\psi_{K}\right\rangle$ pode ser decomposto em seis estados produto de 3 maneiras diferentes,

$$
\begin{aligned}
\left|\psi_{K}\right\rangle & =\frac{1}{\sqrt{2}}\left(\left|\xi_{1}\right\rangle|0\rangle+\left|\xi_{2}\right\rangle|1\rangle\right) \\
& =\frac{1}{\sqrt{2}}\left[\frac{1}{3}\left(2|11\rangle+2\left|a_{1}\right\rangle\left|b_{1}\right\rangle+\left|a_{2}\right\rangle\left|b_{2}\right\rangle\right)|0\rangle+\frac{1}{\sqrt{5}}(|00\rangle-\sqrt{2}(|22\rangle+|33\rangle))|1\rangle\right]
\end{aligned}
$$

ou

$$
\begin{aligned}
\left|\psi_{K}\right\rangle & =\frac{1}{\sqrt{2}}\left[\left|\xi_{1}\right\rangle|0\rangle+\frac{3}{\sqrt{5}}\left(\left|\xi_{1}\right\rangle-\frac{\sqrt{6}}{3}\left|\xi_{3}\right\rangle\right)|1\rangle\right] \\
& =\frac{1}{\sqrt{2}}\left[\left(2|11\rangle+2\left|a_{1}\right\rangle\left|b_{1}\right\rangle+\left|a_{2}\right\rangle\left|b_{2}\right\rangle\right)\left(|0\rangle+\frac{3}{\sqrt{5}}|1\rangle\right)-\frac{1}{\sqrt{5}}(|00\rangle+2|11\rangle+|32\rangle)|1\rangle\right]
\end{aligned}
$$

ou ainda

$$
\begin{aligned}
\left|\psi_{K}\right\rangle & =\frac{1}{\sqrt{2}}\left[\frac{\sqrt{6}}{3}\left(\left|\xi_{3}\right\rangle-\sqrt{\frac{5}{6}}\left|\xi_{2}\right\rangle\right)|0\rangle+\left|\xi_{2}\right\rangle|1\rangle\right] \\
& =\frac{1}{\sqrt{2}}\left[\frac{\sqrt{6}}{3}\left|\xi_{3}\right\rangle|0\rangle+\left|\xi_{2}\right\rangle\left(|1\rangle-\frac{\sqrt{5}}{3}|0\rangle\right)\right] .
\end{aligned}
$$

Este exemplo demonstra as limitações da nossa visualização das correlações quânticas destes estados emaranhados em termos de diagramas. Neste caso não conseguimos encontrar um exemplo tal que todos os estados $\left|\xi_{i}\right\rangle$ possam ser escritos nas mesmas bases de Schmidt nos subsistemas de Alice e Bob. De certa forma, isso mostra as limitações de uma maneira um tanto clássica de tentarmos entender o emaranhamento, pois, quando desenhamos os diagramas, estamos explicitando as correlações existentes em uma determinada base. Assim, nos limitamos a comparar as correlações dos estados $\left|\xi_{2}\right\rangle$ e $\left|\xi_{3}\right\rangle$ no diagrama da Figura 3.16 .

Para mostrarmos que $\left|\psi_{K}\right\rangle$ pertence a família da matriz $K$, escolhemos o estado $\left|\phi_{1}\right\rangle=$ $\sqrt{6}\left|\xi_{1}\right\rangle+\sqrt{5}\left|\xi_{2}\right\rangle$ com posto de Schmidt 4. As matrizes correspondentes ao estado $\left|\phi_{1}\right\rangle$ e 


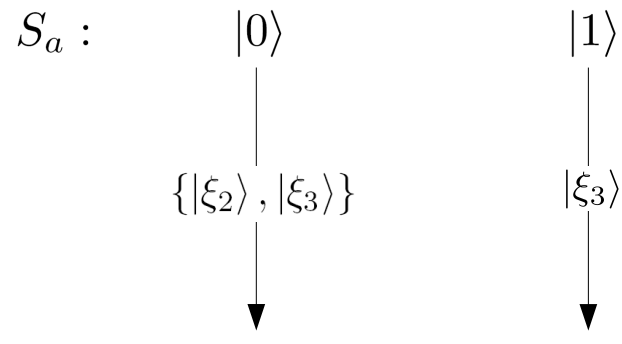

$S_{b}: \quad|0\rangle$ $|1\rangle$

$|1\rangle$ $|2\rangle$

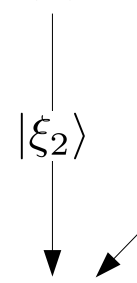

$|2\rangle$ $|3\rangle$

$\left|\xi_{3}\right\rangle$

$\left|\xi_{2}\right\rangle$

$|3\rangle$

Figura 3.16: Diagrama de correlações do estado $\left|\psi_{K}\right\rangle$. Neste caso, comparamos apenas os estados $\left|\xi_{2}\right\rangle$ e $\left|\xi_{3}\right\rangle$.

ao estado $\sqrt{5}\left|\xi_{2}\right\rangle$ são

$$
\Phi_{1}=\left(\begin{array}{cccc}
2 & 0 & 0 & 0 \\
0 & 2 & 0 & 0 \\
0 & 0 & -\sqrt{2} & 1 \\
0 & 0 & 0 & -\sqrt{2}
\end{array}\right) \quad \text { e } \quad \Xi_{2}=\left(\begin{array}{cccc}
1 & 0 & 0 & 0 \\
0 & 0 & 0 & 0 \\
0 & 0 & -\sqrt{2} & 0 \\
0 & 0 & 0 & -\sqrt{2}
\end{array}\right),
$$

respectivamente. Assim temos

$$
\Phi_{1}^{-1} \Xi_{2}=\left(\begin{array}{cccc}
2 & 0 & 0 & 0 \\
0 & 2 & 0 & 0 \\
0 & 0 & -\frac{1}{\sqrt{2}} & -\frac{1}{2} \\
0 & 0 & 0 & -\frac{1}{\sqrt{2}}
\end{array}\right)\left(\begin{array}{cccc}
1 & 0 & 0 & 0 \\
0 & 0 & 0 & 0 \\
0 & 0 & -\sqrt{2} & 0 \\
0 & 0 & 0 & -\sqrt{2}
\end{array}\right)=\left(\begin{array}{cccc}
2 & 0 & 0 & 0 \\
0 & 0 & 0 & 0 \\
0 & 0 & 1 & \frac{1}{\sqrt{2}} \\
0 & 0 & 0 & 1
\end{array}\right)
$$

que é similar a $K \operatorname{com} \lambda_{1}=2, \lambda_{2}=0$ e $\lambda_{3}=1$ e portanto $\left|\psi_{K}\right\rangle$ pertence à família da matriz $K$.

\section{Família L}

Vamos definir a família L como os estados pertencentes a família de Jordan da matriz

$$
L=\left(\begin{array}{cccc}
\lambda_{1} & 0 & 0 & 0 \\
0 & \lambda_{2} & 0 & 0 \\
0 & 0 & \lambda_{3} & 0 \\
0 & 0 & 0 & \lambda_{3}
\end{array}\right)
$$

onde $\lambda_{1} \neq \lambda_{2} \neq \lambda_{3}$.

Para obtermos um exemplo de um estado $\left|\psi_{L}\right\rangle$ pertencente a esta família, mais uma vez, é conveniente escolhermos os estados no suporte local de $\left|\psi_{L}\right\rangle$ no subsistema $S_{a b}$, $\mathcal{P}_{\left|\psi_{L}\right\rangle}$, com posto de Schmidt menor que $n$. Da matriz $L$, sabemos que $\mathcal{P}_{\left|\psi_{L}\right\rangle}$ contém três 
estados com posto de Schmidt menor que $n$. Vamos escolher dois deles,

$$
\begin{aligned}
& \left|\xi_{1}\right\rangle=|11\rangle+|22\rangle+|33\rangle \\
& \left|\xi_{2}\right\rangle=|00\rangle+|22\rangle+|33\rangle,
\end{aligned}
$$

os quais podem ser relacionados com $\lambda_{1}$ e $\lambda_{2}$, respectivamente. O terceiro estado pode ser obtido desses dois,

$$
\left|\xi_{3}\right\rangle=\left|\xi_{1}\right\rangle-\left|\xi_{2}\right\rangle=|00\rangle-|11\rangle
$$

Temos também

$$
\begin{aligned}
\left|\xi_{1}\right\rangle & =\left|\xi_{2}\right\rangle-\left|\xi_{3}\right\rangle \\
\left|\xi_{2}\right\rangle & =\left|\xi_{1}\right\rangle+\left|\xi_{3}\right\rangle
\end{aligned}
$$

Dessa forma, o estado $\left|\psi_{L}\right\rangle$ possui três decomposições sub-Schmidt, duas com cinco estados produto e uma com seis estados produto. São elas:

$$
\left|\psi_{L}\right\rangle=\frac{1}{\sqrt{6}}[(|00\rangle+|22\rangle+|33\rangle)|0\rangle+(|11\rangle+|22\rangle+|33\rangle)|1\rangle]
$$

ou

$$
\begin{aligned}
\left|\psi_{L}\right\rangle & =\frac{1}{\sqrt{6}}\left[\left(\left|\xi_{2}\right\rangle-\left|\xi_{3}\right\rangle\right)|0\rangle+\left|\xi_{2}\right\rangle|1\rangle\right] \\
& =\frac{1}{\sqrt{6}}\left[-\left|\xi_{3}\right\rangle|0\rangle+\left|\xi_{2}\right\rangle|+\rangle\right]
\end{aligned}
$$

onde $|+\rangle=(|0\rangle+|1\rangle)$, ou ainda

$$
\begin{aligned}
\left|\psi_{L}\right\rangle & =\frac{1}{\sqrt{6}}\left[\left|\xi_{1}\right\rangle|0\rangle+\left(\left|\xi_{1}\right\rangle+\left|\xi_{3}\right\rangle\right)|1\rangle\right] \\
& =\frac{1}{\sqrt{6}}\left[\left|\xi_{1}\right\rangle|+\rangle+\left|\xi_{3}\right\rangle|1\rangle\right] .
\end{aligned}
$$

O diagrama de correlações do estado $\left|\psi_{L}\right\rangle$ pode ser visto na Figura 3.17.

Para mostramos que $\left|\psi_{L}\right\rangle$ de fato pertence a família L, precisamos de um estado em $\mathcal{P}_{\left|\psi_{L}\right\rangle}$ com posto de Schmidt 4. Escolhemos $\left|\phi_{1}\right\rangle=\left|\xi_{1}\right\rangle+\left|\xi_{2}\right\rangle$ que corresponde a matriz

$$
\Phi_{1}=\left(\begin{array}{cccc}
1 & 0 & 0 & 0 \\
0 & 1 & 0 & 0 \\
0 & 0 & 2 & 0 \\
0 & 0 & 0 & 2
\end{array}\right) .
$$




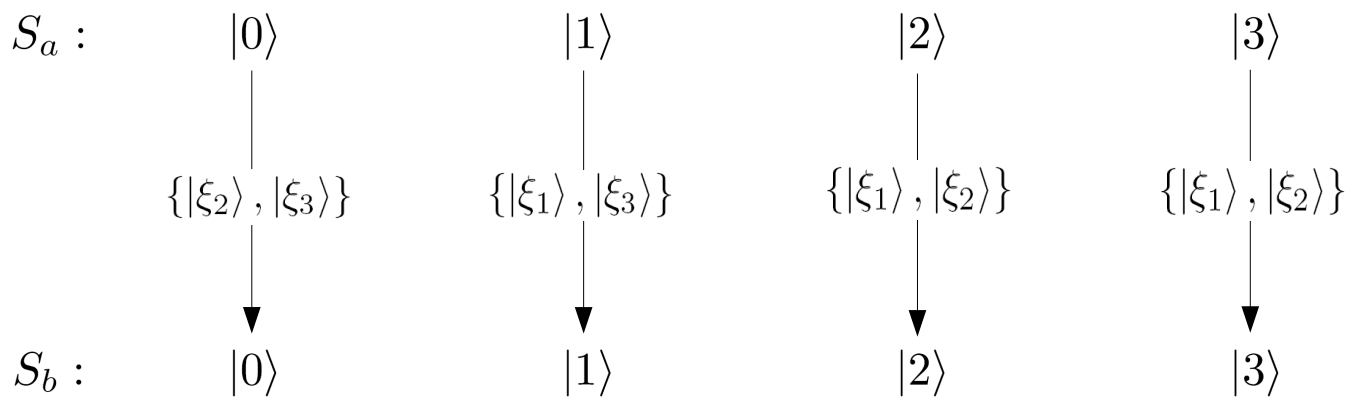

Figura 3.17: Diagrama de correlações do estado $\left|\psi_{L}\right\rangle$. Os estados $|0\rangle$ e $|1\rangle$ e o subespaço gerado por $\{|2\rangle,|3\rangle\}$ possuem uma correlação bem definida de $S_{a}$ para $S_{b}$.

Como a matriz correspondente de $\left|\xi_{1}\right\rangle$ é

$$
\Xi_{1}=\left(\begin{array}{cccc}
0 & 0 & 0 & 0 \\
0 & 1 & 0 & 0 \\
0 & 0 & 1 & 0 \\
0 & 0 & 0 & 1
\end{array}\right)
$$

temos

$$
\Phi_{1}^{-1} \Xi_{1}=\left(\begin{array}{cccc}
0 & 0 & 0 & 0 \\
0 & 1 & 0 & 0 \\
0 & 0 & \frac{1}{2} & 0 \\
0 & 0 & 0 & \frac{1}{2}
\end{array}\right)
$$

que é similar a $L \operatorname{com} \lambda_{1}=0, \lambda_{2}=1$ e $\lambda_{3}=1 / 2$ e portanto $\left|\psi_{L}\right\rangle$ pertence à família L.

\section{Família M}

Vamos definir como a família M os estados correspondentes a família de Jordan da matriz

$$
M=\left(\begin{array}{cccc}
\lambda_{1} & 0 & 0 & 0 \\
0 & \lambda_{2} & 0 & 0 \\
0 & 0 & \lambda_{3} & 0 \\
0 & 0 & 0 & \lambda_{4}
\end{array}\right)
$$

onde $\lambda_{1} \neq \lambda_{2} \neq \lambda_{3} \neq \lambda_{4}$.

Para obtermos um exemplo $\left|\psi_{M}\right\rangle$ para esta família, vamos escolher os estados com posto de Schmidt menor que $n$ no suporte local de $\left|\psi_{M}\right\rangle$ no subsistema $S_{a b}, \mathcal{P}_{\left|\psi_{M}\right\rangle}$. Da matriz $M$, sabemos que $\mathcal{P}_{\left|\psi_{H}\right\rangle}$ contém quatro estados com posto de Schmidt 3. Vamos 
escolher dois deles correspondentes aos autovalores $\lambda_{1}$ e $\lambda_{2}$,

$$
\begin{aligned}
& \left|\xi_{1}\right\rangle=|11\rangle+2|22\rangle+|33\rangle \\
& \left|\xi_{2}\right\rangle=|00\rangle+2|11\rangle+|22\rangle
\end{aligned}
$$

respectivamente. De $\left|\xi_{1}\right\rangle$ e $\left|\xi_{2}\right\rangle$ podemos obter os outros dois estados com posto de Schmidt $3 \mathrm{em} \mathcal{P}_{\left|\psi_{H}\right\rangle}$,

$$
\begin{aligned}
\left|\xi_{3}\right\rangle & =\left|\xi_{2}\right\rangle-\frac{1}{2}\left|\xi_{1}\right\rangle=|00\rangle+\frac{3}{2}|11\rangle-\frac{1}{2}|33\rangle \\
\left|\xi_{4}\right\rangle & =\left|\xi_{1}\right\rangle-\frac{1}{2}\left|\xi_{2}\right\rangle=-\frac{1}{2}|00\rangle+\frac{3}{2}|22\rangle+|33\rangle .
\end{aligned}
$$

Também temos que

$$
\left|\xi_{2}\right\rangle=\frac{4}{3}\left|\xi_{3}\right\rangle+\frac{2}{3}\left|\xi_{4}\right\rangle
$$

Dessa forma, existem quatro estados com posto de Schmidt 3 no plano $\mathcal{P}_{\left|\psi_{M}\right\rangle}$ e, portanto, existem seis diferentes decomposições sub-Schmidt para um estado pertencente a esta família, todas elas com seis estados produtos. São elas:

$$
\left|\psi_{H}\right\rangle=\frac{1}{2 \sqrt{3}}\left[\left|\xi_{1}\right\rangle|0\rangle+\left|\xi_{2}\right\rangle|1\rangle\right]
$$

$\mathrm{Ou}$

$$
\left|\psi_{H}\right\rangle=\frac{1}{2 \sqrt{3}}\left[\left|\xi_{1}\right\rangle|0\rangle+\left(\left|\xi_{3}\right\rangle+\frac{1}{2}\left|\xi_{1}\right\rangle\right)|1\rangle\right]=\frac{1}{2 \sqrt{3}}\left[\left|\xi_{1}\right\rangle\left(|0\rangle+\frac{1}{2}|1\rangle\right)+\left|\xi_{3}\right\rangle|1\rangle\right]
$$

$\mathrm{ou}$

$$
\left|\psi_{H}\right\rangle=\frac{1}{2 \sqrt{3}}\left[\left|\xi_{1}\right\rangle|0\rangle+\left(-2\left|\xi_{4}\right\rangle+2\left|\xi_{1}\right\rangle\right)|1\rangle\right]=\frac{1}{2 \sqrt{3}}\left[\left|\xi_{1}\right\rangle(|0\rangle+2|1\rangle)+-2\left|\xi_{4}\right\rangle|1\rangle\right]
$$

$\mathrm{ou}$

$$
\left|\psi_{H}\right\rangle=\frac{1}{2 \sqrt{3}}\left[\left(-2\left|\xi_{3}\right\rangle+2\left|\xi_{2}\right\rangle\right)|0\rangle+\left|\xi_{2}\right\rangle|1\rangle\right]=\frac{1}{2 \sqrt{3}}\left[\left|\xi_{2}\right\rangle(2|0\rangle+|1\rangle)-2\left|\xi_{3}\right\rangle|0\rangle\right]
$$

$\mathrm{ou}$

$$
\left|\psi_{H}\right\rangle=\frac{1}{2 \sqrt{3}}\left[\left(\left|\xi_{4}\right\rangle+\frac{1}{2}\left|\xi_{2}\right\rangle\right)|0\rangle+\left|\xi_{2}\right\rangle|1\rangle\right]=\frac{1}{2 \sqrt{3}}\left[\left|\xi_{2}\right\rangle\left(\frac{1}{2}|0\rangle+|1\rangle\right)+\left|\xi_{4}\right\rangle|0\rangle\right]
$$




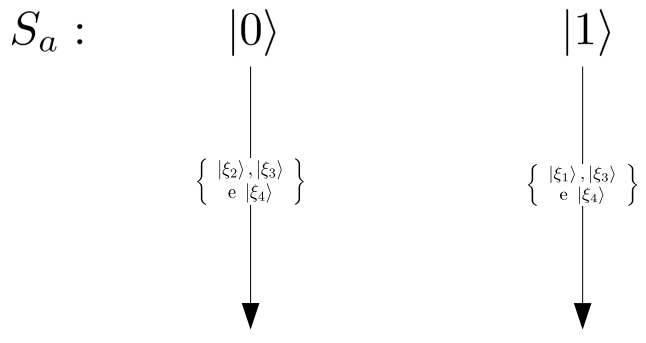

$S_{b}:$

$|0\rangle$ $|2\rangle$

$|3\rangle$

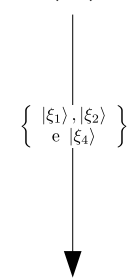

$|2\rangle$

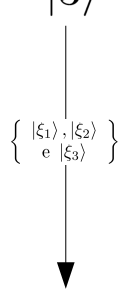

$|3\rangle$

Figura 3.18: Diagrama de correlações do estado $\left|\psi_{M}\right\rangle$. Os estados $|0\rangle,|1\rangle,|2\rangle$ e $|3\rangle$ possuem correlações bem definidas de $S_{a}$ para $S_{b}$.

ou finalmente

$$
\begin{aligned}
\left|\psi_{H}\right\rangle= & \frac{1}{2 \sqrt{3}}\left[\left(\left|\xi_{4}\right\rangle+\frac{1}{2}\left(\frac{4}{3}\left|\xi_{3}\right\rangle+\frac{2}{3}\left|\xi_{4}\right\rangle\right)\right)|0\rangle+\left(\frac{4}{3}\left|\xi_{3}\right\rangle+\frac{2}{3}\left|\xi_{4}\right\rangle\right)|1\rangle\right] \\
= & \frac{1}{2 \sqrt{3}}\left[\left|\xi_{3}\right\rangle\left(\frac{2}{3}|0\rangle+\frac{4}{3}|1\rangle\right)+\left|\xi_{4}\right\rangle\left(\frac{5}{3}|0\rangle+\frac{2}{3}|1\rangle\right)\right]
\end{aligned}
$$

O diagrama de correlações do estado $\left|\psi_{M}\right\rangle$ pode ser visto na Figura 3.18.

Como veremos na seção 3.3, esta família subdivide-se em uma infinidade de classes não equivalentes através de SLOCC. Nesta dimensionalidade, $(4,4,2)$, esta família é a única que não corresponde a uma única classe de estados sobre SLOCC, pois é a única com quatro autovalores distintos. Como veremos na 3.3, isso será uma propriedade de todas as famílias com mais do que três autovalores distintos. A existência de uma infinidade de classes SLOCC para estados emaranhado de dimensionalidade maior já havia sido observada por Dür et al [17] através de um argumento de contagem de parâmetros. Porém, este é o primeiro exemplo bem caracterizado de uma classe deste tipo.

Para mostrarmos que $\left|\psi_{H}\right\rangle$ de fato pertence a família M, vamos utilizar os estados $\left|\xi_{1}\right\rangle$ e $\left|\phi_{1}\right\rangle=\left|\xi_{1}\right\rangle+\left|\xi_{2}\right\rangle$ correspondentes às matrizes

$$
\Xi_{1}=\left(\begin{array}{cccc}
0 & 0 & 0 & 0 \\
0 & 1 & 0 & 0 \\
0 & 0 & 2 & 0 \\
0 & 0 & 0 & 1
\end{array}\right) \quad \text { e } \quad \Phi_{1}=\left(\begin{array}{cccc}
1 & 0 & 0 & 0 \\
0 & 3 & 0 & 0 \\
0 & 0 & 3 & 0 \\
0 & 0 & 0 & 1
\end{array}\right)
$$

respectivamente. Assim, temos

$$
\Phi_{1}^{-1} \Xi_{1}=\left(\begin{array}{cccc}
0 & 0 & 0 & 0 \\
0 & \frac{1}{3} & 0 & 0 \\
0 & 0 & \frac{2}{3} & 0 \\
0 & 0 & 0 & 1
\end{array}\right)
$$




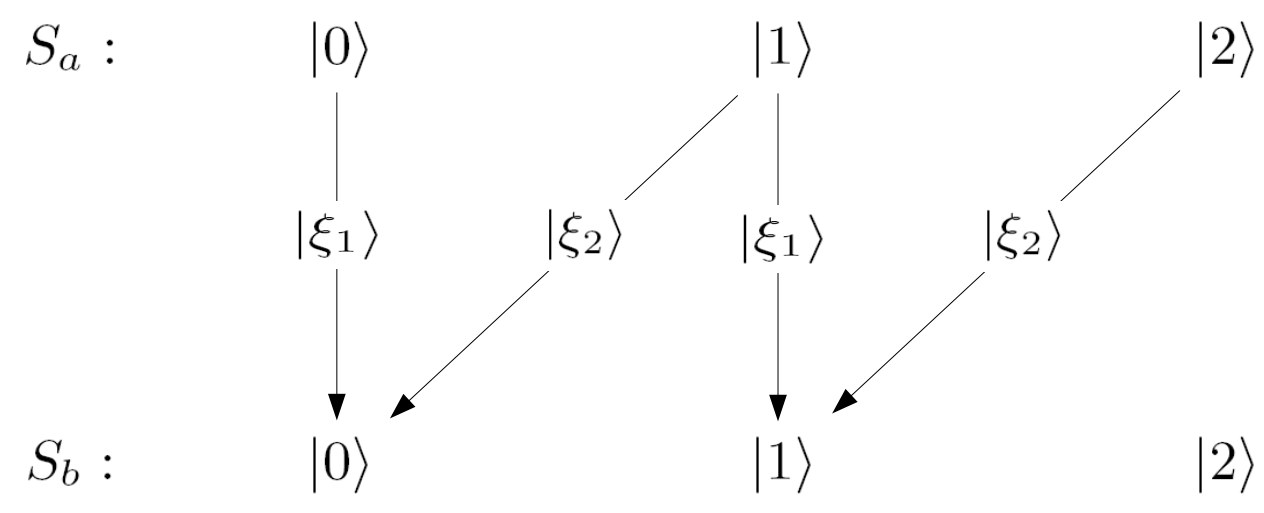

Figura 3.19: Diagrama de correlações do estado $|\psi\rangle$. A imagem de qualquer combinação linear de $\left|\xi_{1}\right\rangle$ e $\left|\xi_{2}\right\rangle$, isto é, qualquer estado em $\mathcal{P}_{|\psi\rangle}$, será o subespaço gerado por $\{|0\rangle,|1\rangle\}$.

que é similar à matriz $M$ com autovalores $\lambda_{1}=0, \lambda_{2}=1 / 3, \lambda_{3}=1 / 3$ e $\lambda_{4}=1$ e portanto $\left|\psi_{M}\right\rangle$ pertence a família $M$.

\subsubsection{Contra-exemplos}

\section{Contra-exemplo A}

O objetivo deste exemplo é mostrar porque o método descrito na seção 3.1 não funciona para estados emaranhados de dimensionalidade $(m, n, 2)$, com $m \neq n$. Vamos considerar o estado

$$
|\psi\rangle=\frac{1}{2}((|00\rangle+|11\rangle)|0\rangle+(|10\rangle+|21\rangle)|1\rangle)
$$

de dimensionalide $(3,2,2)$. A Figura 3.19 mostra o diagrama de correlações de $|\psi\rangle$.

O suporte local de $|\psi\rangle$ no subsistema $S_{a b}, \mathcal{P}_{|\psi\rangle}$, é gerado pelos estados

$$
\left|\xi_{1}\right\rangle=|00\rangle+|11\rangle
$$

e

$$
\left|\xi_{2}\right\rangle=|10\rangle+|21\rangle
$$

Esses dois estados correspondem às matrizes

$$
\Xi_{1}=\left(\begin{array}{ccc}
1 & 0 & 0 \\
0 & 1 & 0 \\
0 & 0 & 0
\end{array}\right) \quad \text { e } \quad \Xi_{2}=\left(\begin{array}{ccc}
0 & 1 & 0 \\
0 & 0 & 1 \\
0 & 0 & 0
\end{array}\right)
$$

Dessa forma, qualquer combinação linear de $\Xi_{1}$ e $\Xi_{2}$ resultará em uma matriz com a terceira linha identicamente nula e, portanto, não inversível. Assim, qualquer combinação linear dos estados $\left|\xi_{1}\right\rangle$ e $\left|\xi_{2}\right\rangle$ também terá posto de Schmidt 2. Isso acontece porque 


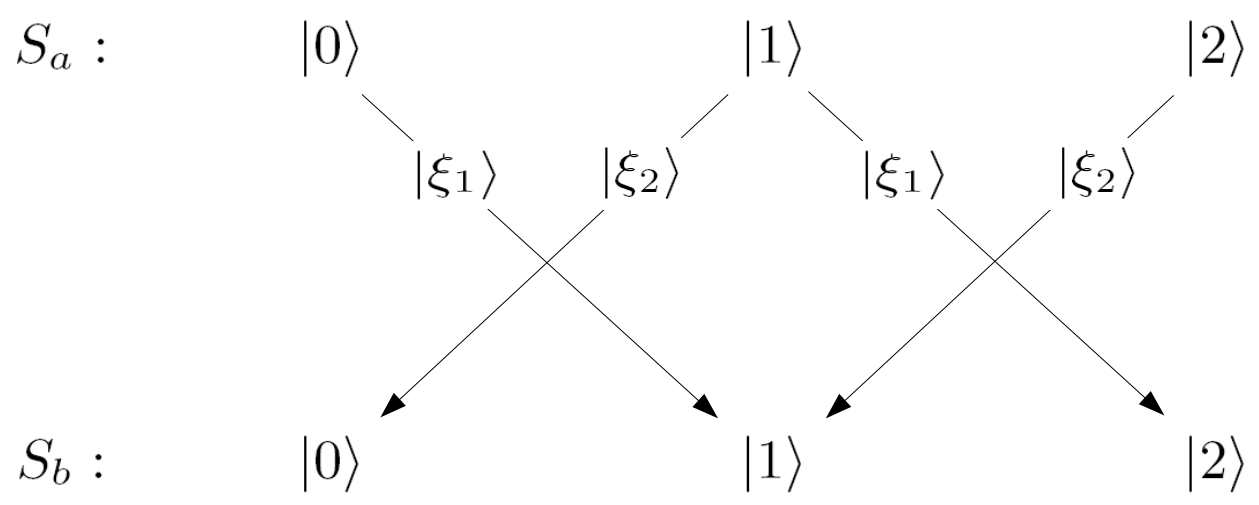

Figura 3.20: Diagrama de correlações do estado $|\psi\rangle$.

qualquer estado $|\phi\rangle$ em $\mathcal{P}_{|\psi\rangle}$, vistos como uma aplicação linear de $C_{a}^{3 *}$ em $C_{b}^{3}$, possui uma imagem bi-dimensional (veja a Figura 3.19) e portanto corresponde a uma matriz com posto 2 .

Para um estado arbitrário $|\psi\rangle$ de dimensionalidade $(m, n, 2)$, onde $m>n$ (podemos supor sem perda de generalidade que $m>n$ ), qualquer estado $|\phi\rangle$ no suporte local de $|\psi\rangle$ no subsistema $S_{a b}$ terá posto de Schmidt no máximo $n$ e suas respectivas matrizes $m \times m$ terão $m-n$ linhas identicamente nulas. Dessa forma, temos que o método das decomposições sub-Schmidt da seção 3.1 não pode ser aplicado para estados de dimensionalidade $(m, n, 2)$, para $m \neq n$.

\section{Contra-exemplo B}

Neste exemplo, vamos mostrar que também existem estados de dimensionalidade $(n, n, 2)$ para os quais não é possível aplicarmos o método das decomposições sub-Schmidt. Vamos considerar um estado $|\psi\rangle$ de dimensionalidade $(3,3,2)$, tais que o suporte local no subsistema $S_{a b}, \mathcal{P}_{|\psi\rangle}$, contenha apenas estados com posto de Schmidt 2.

Escolhemos $|\psi\rangle$ como

$$
|\psi\rangle=\frac{1}{2}[(|01\rangle+|12\rangle)|0\rangle+(|10\rangle+|21\rangle)|1\rangle] .
$$

A Figura 3.19 mostra o diagrama de correlações de $|\psi\rangle$. Nesse caso, $\mathcal{P}_{|\psi\rangle}$ é gerado pelos estados

$$
\left|\xi_{1}\right\rangle=|01\rangle+|12\rangle \quad \text { e } \quad\left|\xi_{2}\right\rangle=|10\rangle+|21\rangle,
$$

os quais correspondem às matrizes

$$
\Xi_{1}=\left(\begin{array}{ccc}
0 & 1 & 0 \\
0 & 0 & 1 \\
0 & 0 & 0
\end{array}\right) \quad \text { e } \quad \Xi_{2}=\left(\begin{array}{ccc}
0 & 0 & 0 \\
1 & 0 & 0 \\
0 & 1 & 0
\end{array}\right)
$$


Dessa forma, temos que qualquer combinação linear das matrizes $\Xi_{1}$ e $\Xi_{2}$ terá a primeira e a terceira linha linearmente dependentes e, portanto, corresponderá a um estado com posto de Schmidt 2.

Além disso, similarmente, podemos construir um estado $|\psi\rangle$ de dimensionalidade $(n, n, 2)$, tal que o suporte local de $|\psi\rangle$ no subsistema $S_{a b}$ contenha estados similares a $\left|\phi_{1}\right\rangle$ e $\left|\phi_{2}\right\rangle$, os quais corresponderiam às matrizes

$$
\Phi_{1}^{(n)}=\left(\begin{array}{cccccc}
0 & 0 & \cdots & 0 & 1 & 0 \\
0 & 0 & \cdots & \cdots & 0 & 1 \\
0 & 0 & 0 & & & 0 \\
\vdots & \vdots & & \ddots & & 0 \\
\vdots & \vdots & & & \ddots & \vdots \\
0 & 0 & \cdots & \cdots & \cdots & 0
\end{array}\right) \quad \text { e } \quad \Phi_{2}^{(n)}\left(\begin{array}{ccccc}
0 & & & & \\
1 & 0 & & & \\
& 1 & 0 & & \\
& & \ddots & \ddots & \\
& & & 1 & 0
\end{array}\right) .
$$

Dessa forma, também teremos que qualquer combinação linear de $\Phi_{1}^{(n)}$ e $\Phi_{2}^{(n)}$ terá a primeira e a última linha linearmente dependentes e portanto existem estados de dimensionalidade $(n, n, 2)$ para os quais não podemos aplicar o método das decomposições sub-Schmidt.

\subsection{Decomposições sub-Schmidt e operações do tipo SLOCC}

Nesta seção, vamos discutir a relação entre as decomposições sub-Schmidt desenvolvidas na seção 3.1 e as transformações dos estados emaranhados através de SLOCC. Vamos começar demonstrando o Lema 3.1, o qual relaciona as matrizes $R_{1}^{-1} R_{0}$ da eq. (3.5) derivadas de dois estados distintos pertencentes a uma mesma classe SLOCC. Depois, com uma analise da relação entre os autovalores dada pela eq. (3.6) no Teorema 3.1, obtemos um critério prático para verificarmos se dois estados, para os quais podemos aplicar o método das decomposições sub-Schmidt, pertencem a uma mesma classe de estados emaranhados por SLOCC. Esse é o principal resultado desta seção e é expresso pelo Teorema 3.2. Além disso, será possível identificarmos os operadores lineares inversíveis que relacionam esses dois estados, os quais permitem construir a operação do tipo SLOCC que relaciona estes estados como vimos na seção 2.2 .

Lema 3.1: dois estados emaranhados de dimensionalidade $(n, n, 2)$ para os quais podemos aplicar o método das decomposiçôes sub-Schmidt, $|\psi\rangle$ e $\left|\psi^{\prime}\right\rangle$, são interconversiveis por SLOCC se, e somente se, para qualquer base $\left\{\left|\phi_{0}\right\rangle,\left|\phi_{1}\right\rangle\right\},\left|\phi_{1}\right\rangle$ com posto de Schmidt $n$, para o suporte local de $|\psi\rangle$ no subsistema $S_{a b}$, existe também uma base $\left\{\left|\phi_{k}^{\prime}\right\rangle\right\}$ para o suporte local de $\left|\psi^{\prime}\right\rangle$ no subsistema $S_{a b}$ tal que as respectivas matrizes $\Phi_{1}^{-1} \Phi_{0}$ e $\Phi_{1}^{\prime-1} \Phi_{0}^{\prime}$ são similares.

Prova: $(\Rightarrow)$ Nós sabemos do Teorema 2.2 da seção 2.2 .3 que se $|\psi\rangle$ e $\left|\psi^{\prime}\right\rangle$ pertencem 
à mesma classe SLOCC, então existem operadores lineares inversíveis $A, B$ e $C$ tais que

$$
\left|\psi^{\prime}\right\rangle=A \otimes B \otimes C|\psi\rangle
$$

Podemos expandir $|\psi\rangle$ em uma base qualquer escolhendo dois estados $\left|\phi_{0}\right\rangle$ e $\left|\phi_{1}\right\rangle,\left|\phi_{1}\right\rangle$ com posto de Schmidt $n$, pertencentes ao suporte local de $|\psi\rangle$ no subsistema $S_{a b}, \mathcal{P}_{|\psi\rangle}$, e os respectivos estados $\left|c_{1}\right\rangle$ e $\left|c_{2}\right\rangle$ como fizemos para obter a decomposição sub-Schmidt na eq. (3.8) da seção 3.1. Assim temos

$$
\left|\psi^{\prime}\right\rangle=\sum_{k=0,1} A \otimes B\left|\phi_{k}\right\rangle C\left|c_{k}\right\rangle=\sum_{k=0,1}\left|\phi_{k}^{\prime}\right\rangle\left|c_{k}^{\prime}\right\rangle
$$

onde $\left|c_{k}^{\prime}\right\rangle=C\left|c_{k}\right\rangle$ e $\left|\phi_{k}^{\prime}\right\rangle=A \otimes B\left|\phi_{k}\right\rangle$. Uma vez que não normalizamos os operadores $A$, $B$ e $C$, os estados $\left|c_{k}^{\prime}\right\rangle$ e $\left|\phi_{k}^{\prime}\right\rangle$ e conseqüentemente $\left|\psi^{\prime}\right\rangle$ também não estão normalizados.

Primeiramente, devemos notar que sempre existe um operador inversível $C$ em um espaço linear complexo $C_{c}^{2}$ que mapeia quaisquer dois estados $\left|c_{0}\right\rangle$ e $\left|c_{1}\right\rangle$ distintos em outros dois quaisquer estados distintos $\left|c_{0}^{\prime}\right\rangle$ e $\left|c_{1}^{\prime}\right\rangle$. Então o operador $C$ não impõe nenhuma restrição aos estados $|\psi\rangle$ e $\left|\psi^{\prime}\right\rangle$.

Vamos então analisar a ação do operador $A \otimes B$ nos estados $\left|\phi_{0}\right\rangle$ e $\left|\phi_{1}\right\rangle$ e, portanto, em $\mathcal{P}_{|\psi\rangle}$. Para cada estado $|\phi\rangle$ pertencente a $\mathcal{P}_{|\psi\rangle}$, existe um único estado $\left|\phi^{\prime}\right\rangle$ pertencente a $\mathcal{P}_{|\psi\rangle}^{\prime}$ tal que $\left|\phi^{\prime}\right\rangle=A \otimes B|\phi\rangle$. Definindo uma base $\{|i\rangle\}$ para $C_{a}^{n}$ e uma base $\{|j\rangle\}$ para $C_{b}^{n}$, as matrizes dos operadores $A$ e $B$ ficam com as seguintes componentes

$$
A_{i^{\prime} i}=\left\langle i^{\prime}|A| i\right\rangle, \quad B_{j^{\prime} j}=\left\langle j^{\prime}|B| j\right\rangle
$$

respectivamente. Também temos as componentes

$$
\left[\Phi_{k}\right]_{j i}=\left\langle i j \mid \phi_{k}\right\rangle \quad \text { e } \quad\left[\Phi_{k}^{\prime}\right]_{j i}=\left\langle i j \mid \phi_{k}^{\prime}\right\rangle
$$

para as matrizes $\Phi_{k}$ e $\Phi_{k}^{\prime}, k=0,1$, correspondentes aos estados $\left|\phi_{k}\right\rangle$ e $\left|\phi_{k}^{\prime}\right\rangle$. Dessa forma a relação entre os estados

$$
\left|\phi_{k}^{\prime}\right\rangle=A \otimes B\left|\phi_{k}\right\rangle
$$

fica, em forma matricial,

$$
\Phi_{k}^{\prime}=B \Phi_{k} A^{T}
$$

Então, temos que as matrizes $\Phi_{1}^{-1} \Phi_{0}$ e $\Phi_{1}^{\prime-1} \Phi_{0}^{\prime}$ são relacionadas através da expressão

$$
\begin{aligned}
\Phi_{1}^{\prime-1} \Phi_{0}^{\prime} & =\left(B \Phi_{1} A^{T}\right)^{-1} B \Phi_{0} A^{T} \\
& =A^{T^{-1}} \Phi_{1}^{-1} \Phi_{0} A^{T}
\end{aligned}
$$


isto é, as matrizes $\Phi_{1}^{-1} \Phi_{0}$ e $\Phi_{1}^{\prime-1} \Phi_{0}^{\prime}$ são similares.

$(\Leftarrow)$ Uma questão natural neste ponto é: o que aconteceu com operador $B$ ? O fato de ele ter desaparecido na eq. (3.14) não pode significar que $B$ é irrelevante para a transformação. De fato, se invertermos o papel dos subsistemas de Alice e Bob, ao invés da eq. (3.14), vamos obter a equação

$$
\left(\Phi_{0}^{\prime} \Phi_{1}^{\prime-1}\right)^{T}=B^{T^{-1}}\left(\Phi_{0} \Phi_{1}^{-1}\right)^{T} B^{T}
$$

onde é o operador $A$ que desaparece. Como as matrizes $\Phi_{0}^{\prime} \Phi_{1}^{\prime-1}$ e $\Phi_{0} \Phi_{1}^{-1}$ são similares se, e somente se, as matrizes $\left(\Phi_{1}^{-1} \Phi_{0}\right)^{T}$ e $\left(\Phi_{1}^{\prime-1} \Phi_{0}^{\prime}\right)^{T}$ também são, temos que, se $\Phi_{1}^{-1} \Phi_{0}$ e $\Phi_{1}^{\prime-1} \Phi_{0}^{\prime}$ são similares, existe um operador linear inversível $A$ definido pela eq. (3.14), um operador linear inversível $B$ definido pela eq. (3.15) e um operador linear inversível $C$ mapeando os estados $\left|c_{0}\right\rangle$ e $\left|c_{1}\right\rangle$ nos estados $\left|c_{0}^{\prime}\right\rangle$ e $\left|c_{1}^{\prime}\right\rangle$, respectivamente tal que a eq. (3.12) é satisfeita e os estados $\left|\psi^{\prime}\right\rangle$ e $|\psi\rangle$ pertence a mesma classe SLOCC.

Do Teorema 3.1 e do Lema 3.1, temos que dois estados, $|\psi\rangle$ e $\left|\psi^{\prime}\right\rangle$, pertencem à mesma classe SLOCC somente se pertencem à mesma família de Jordan. No entanto, se $|\psi\rangle$ e $\left|\psi^{\prime}\right\rangle$ pertencem à mesma família de Jordan podemos utilizar o Lema 3.1 para verificarmos se eles também pertence à mesma classe SLOCC. Porém, o Lema 3.1 não nos fornece um método prático para encontrarmos as bases dos respectivos suportes locais, $\mathcal{P}_{|\psi\rangle}$ e $\mathcal{P}_{|\psi\rangle}^{\prime}$, tais que as matrizes $\Phi_{1}^{-1} \Phi_{0}$ e $\Phi_{1}^{\prime-1} \Phi_{0}^{\prime}$ sejam similares. Assim sendo, o Teorema 3.2 a seguir nos dá um critério simples para sabermos se dois estados $|\psi\rangle$ e $\left|\psi^{\prime}\right\rangle$ pertencem a mesma classe SLOCC. A demonstração do teorema é um método para encontrarmos essas bases tais que as matrizes $\Phi_{1}^{-1} \Phi_{0}$ e $\Phi_{1}^{\prime-1} \Phi_{0}^{\prime}$ são similares.

Teorema 3.2: Sejam $|\psi\rangle$ e $\left|\psi^{\prime}\right\rangle$ dois estados puros emaranhados, para os quais podemos aplicar o método das decomposiçôes sub-Schmidt, e $\left\{\left|\phi_{0}\right\rangle,\left|\phi_{1}\right\rangle\right\}$ e $\left\{\left|\phi_{0}^{\prime}\right\rangle,\left|\phi_{1}^{\prime}\right\rangle\right\}$ bases arbitrárias para os respectivos suportes locais de $|\psi\rangle$ e $\left|\psi^{\prime}\right\rangle$ no subsistema $S_{a b}$. Sejam também $\Phi_{0}, \Phi_{1}, \Phi_{0}^{\prime}$ e $\Phi_{1}^{\prime}$ as matrizes correspondentes aos estados $\left|\phi_{0}\right\rangle,\left|\phi_{1}\right\rangle,\left|\phi_{0}^{\prime}\right\rangle$ e $\left|\phi_{1}^{\prime}\right\rangle$, respectivamente. Então, $|\psi\rangle$ e $\left|\psi^{\prime}\right\rangle$ pertencem à mesma classe de estados emaranhados por SLOCC se, somente se, as matrizes $\Phi_{1}^{-1} \Phi_{0}$ e $\Phi_{1}^{\prime-1} \Phi_{0}^{\prime}$ pertencerem à mesma familia de Jordan e:

(a) $\Phi_{1}^{-1} \Phi_{0}$ tem três ou menos autovalores distintos; ou

(b) o sistema de equações lineares

$$
\lambda_{l} \lambda_{l}^{\prime} c+\lambda_{l} d-\lambda_{l}^{\prime} a-b=0,
$$

tem uma solução não-trivial $\{a, b, c, d\}$, onde $\lambda_{l}$ e $\lambda_{l}^{\prime}, l=1,2, \ldots, L$, são os $L$ autovalores das matrizes $\Phi_{1}^{-1} \Phi_{0}$ e $\Phi_{1}^{\prime-1} \Phi_{0}^{\prime}$, respectivamente, e a indexação l é tal que

$$
\text { posto }\left(\Phi_{1}^{-1} \Phi_{0}-\lambda_{l}\right)^{k}=\text { posto }\left(\Phi_{1}^{\prime-1} \Phi_{0}^{\prime}-\lambda_{l}^{\prime}\right)^{k}
$$

para todo $k \geq 1$. Se houver mais de uma forma de indexar os autovalores, basta que o 
sistema de equaçôes lineares (3.16) tenha solução para uma das possiveis indexações.

Prova: $(\Leftrightarrow)$ Pelo Lema 3.1, temos que $|\psi\rangle$ e $\left|\psi^{\prime}\right\rangle$ são equivalentes por SLOCC se, e somente se, pudermos encontrar duas matrizes $\Phi_{1}^{\prime \prime}$ e $\Phi_{0}^{\prime \prime}$, tais que: $\Phi_{1}^{\prime \prime}$ e $\Phi_{0}^{\prime \prime}$ são combinações lineares e independentes de $\Phi_{1}^{\prime}$ e $\Phi_{0}^{\prime}$, isto é,

$$
\begin{aligned}
& \Phi_{0}^{\prime \prime}=a \Phi_{0}^{\prime}+b \Phi_{1}^{\prime} \\
& \Phi_{1}^{\prime \prime}=c \Phi_{0}^{\prime}+d \Phi_{1}^{\prime}
\end{aligned}
$$

com $(a d-b c) \neq 0$; e $\Phi_{1}^{-1} \Phi_{0}$ e $\Phi_{1}^{\prime \prime-1} \Phi_{0}^{\prime \prime}$ são similares. Como $\Phi_{1}^{-1} \Phi_{0}$ e $\Phi_{1}^{\prime \prime-1} \Phi_{0}^{\prime \prime}$ pertencem à mesma família de Jordan, temos que $\Phi_{1}^{-1} \Phi_{0}$ e $\Phi_{1}^{\prime \prime-1} \Phi_{0}^{\prime \prime}$ serão similares se, e somente se, os autovalores de $\Phi_{1}^{\prime \prime-1} \Phi_{0}^{\prime \prime}, \lambda_{l}^{\prime \prime}$, foram idênticos aos de $\Phi_{1}^{-1} \Phi_{0}, \lambda_{l}$, com a indexação $l$ obedecendo à condição da eq. (3.17). Podem existir diversas indexações $l$ satisfazendo a eq. (3.17). Para cada uma delas o procedimento a seguir pode ser aplicado para verificarmos se $\Phi_{1}^{\prime \prime-1} \Phi_{0}^{\prime \prime}$ pode ter autovalores $\lambda_{l}^{\prime \prime}$ idênticos a $\lambda_{l}$ obedecendo (3.17).

Os autovalores $\lambda_{l}^{\prime \prime}$ estão relacionados com os autovalores $\lambda_{l}^{\prime}$ de $\Phi_{1}^{\prime-1} \Phi_{0}^{\prime}$ pela eq. (3.6) do Teorema 3.1. Assim, $\Phi_{1}^{-1} \Phi_{0}$ e $\Phi_{1}^{\prime \prime-1} \Phi_{0}^{\prime \prime}$ serão similares se, e somente se, existirem $a, b$, $c$ e $d$ satisfazendo

$$
\lambda_{l}=\lambda_{l}^{\prime \prime}=\frac{a \lambda_{l}^{\prime}+b}{c \lambda_{l}^{\prime}+d}
$$

Como $c \lambda_{l}^{\prime}+d \neq 0$, pois é um autovalor de $\Phi_{1}^{\prime \prime}$, podemos reescrever essa expressão como

$$
\lambda_{l} \lambda_{l}^{\prime} c+\lambda_{l} d-\lambda_{l}^{\prime} a-b=0
$$

Dessa forma, se $\Phi_{1}^{-1} \Phi_{0}$ tem $L$ autovalores, temos um sistema linear de $L$ equações para $a, b, c$ e $d$. Além disso, para que $\Phi_{0}^{\prime \prime}$ e $\Phi_{1}^{\prime \prime}$ sejam linearmente independentes em (3.18), devemos ter $(a d-b c) \neq 0$ e podemos desconsiderar imediatamente a solução trivial de (3.19).

Agora mostraremos que sempre que pudermos achar uma solução não-trivial, podemos achar uma solução tal que $(a d-b c) \neq 0$.

Primeiro, vamos considerar o caso $L=1$ em que a família de Jordan de $\Phi_{1}^{-1} \Phi_{0}$ tem apenas um autovalor. Neste caso, sempre existem soluções não-triviais da eq. (3.19) tais que $(a d-b c)=0$, as quais devemos desconsiderar, e soluções tais que $(a d-b c) \neq 0$, as quais podem ser facilmente obtidas fazendo $b=c=0 \mathrm{e}$

$$
\frac{\lambda_{1}}{\lambda_{1}^{\prime}}=\frac{a}{d}
$$

No caso $L \geq 2$, qualquer solução não-trivial é tal que $(a d-b c) \neq 0$. Para vermos isso, 
vamos escolher duas das $L$ equações em (3.19),

$$
\left\{\begin{array}{l}
\lambda_{1} \lambda_{1}^{\prime} c+\lambda_{1} d-\lambda_{1}^{\prime} a-b=0 \\
\lambda_{2} \lambda_{2}^{\prime} c+\lambda_{2} d-\lambda_{2}^{\prime} a-b=0
\end{array} .\right.
$$

Então, vamos supor por absurdo que exista uma solução não-trivial tal que $(a d-b c)=0$. Assim temos que $\left(a \lambda_{l}^{\prime}+b\right)$ e $\left(c \lambda_{l}^{\prime}+d\right)$ são linearmente dependentes e diferem por uma constante complexa multiplicativa $K$. Assim, essas duas equações ficam

$$
\left\{\begin{array}{l}
\lambda_{1} K\left(\lambda_{1}^{\prime} a+b\right)-\lambda_{1}^{\prime} a-b=0 \\
\lambda_{2} K\left(\lambda_{2}^{\prime} a+b\right)-\lambda_{2}^{\prime} a-b=0
\end{array}\right.
$$

Da primeira equação, obtemos que

$$
\lambda_{1}=\frac{1}{K}
$$

e da segunda, obtemos também que

$$
\lambda_{2}=\frac{1}{K}
$$

e portanto teríamos $\lambda_{1}=\lambda_{2}$, isto é uma contradição pois $\lambda_{1} \neq \lambda_{2}$. Portanto, qualquer solução não-trivial do sistema linear (3.19) com duas ou mais equações será tal que $(a d-b c) \neq 0$.

Dessa forma, sempre que existir um solução não-trivial do sistema linear de eq. (3.19), poderemos encontrar uma combinação linear (3.18) tal que os autovalores $\lambda_{l}^{\prime \prime}$ sejam idênti$\cos \lambda_{l}$ e as matrizes $\Phi_{1}^{-1} \Phi_{0}$ e $\Phi_{1}^{\prime \prime-1} \Phi_{0}^{\prime \prime}$ sejam similares e, portanto, pelo Lema 3.1, os estados $|\psi\rangle$ e $\left|\psi^{\prime}\right\rangle$ pertencerão à mesma classe SLOCC. Além disso, quando houver mais de uma indexação $l$ possível satisfazendo a condição (3.17), basta que o sistema linear (3.19) tenha solução para uma das indexações. Isso prova o ítem (b). O ítem (a) segue do fato de que o sistema sempre tem uma solução não trivial para $L \leq 3$.

Este teorema nós dá um critério fácil para verificarmos se dois estados são equivalentes por SLOCC. Além disso, as demonstrações do Lema 3.1 e do Teorema 3.2 são construtivas e nos permitem encontrar os operadores lineares lineares $A, B$ e $C$ que relacionam dois estados equivalentes por SLOCC. Assim, podemos encontrar também as operações do tipo SLOCC que os relacionam de maneira fácil.

\subsection{Discussão: Estados emaranhados tri-partidos mais gerais}

Nesta seção, discutiremos a dificuldade de generalizarmos o método das decomposições sub-Schmidt da seção 3.1 para estados emaranhados tri-partidos de maior dimensionalidade. Nesse caso, embora seja fácil obtermos uma equação similar à eq. (3.4), aparentemente é muito difícil resolvermos esta ou classificarmos as possíveis soluções. No caso 
em que um dos subsistemas era um qubit, tivemos a vantagem de podermos comparar a eq. (3.4) a um problema de autovalores. No caso geral, entretanto, somos levados a um sistema de equações polinomiais em muitas variáveis.

Para ilustrarmos essa dificuldade, consideraremos um estado emaranhado $|\psi\rangle$ de dimensionalidade $(n, n, n)$. Usando o raciocínio da seção 3.1, obtemos que a soma na eq. (3.1) tem agora $n$ termos, uma vez que o suporte local de $|\psi\rangle$ no subsistema $S_{a b}, \mathcal{P}_{|\psi\rangle}$, é um hiperplano $n$-dimensional contido em $C_{a}^{n} \otimes C_{b}^{n}$. Nós podemos obter facilmente uma base para $\mathcal{P}_{|\psi\rangle}$ utilizando uma decomposição em estados relativos como fizemos na eq. (3.1), isto é, para qualquer estado $|\phi\rangle$ pertencente a $\mathcal{P}_{|\psi\rangle}$,

$$
|\phi\rangle=\sum_{k} \alpha_{k}\left|r_{k}\right\rangle
$$

onde $\alpha_{k}$ são coeficientes complexos da expansão de $|\phi\rangle$ e $\left|r_{k}\right\rangle$ é o estado relativo de $|k\rangle$, $\{|k\rangle\}$ é uma base ortonormal para $C_{c}^{n}$. Escolhendo uma base $\{|i\rangle\}$ para $C_{a}^{n}$ e uma base $\{|j\rangle\}$ para $C_{b}^{n}$, podemos escrever uma equação matricial similar à eq. (3.4), mas com $n$ matrizes

$$
\left(\sum_{k} \alpha_{k} R_{k}\right) u_{a}^{*}=0
$$

onde as matrizes $R_{k}$ têm componentes $\left[R_{k}\right]_{j i}=\left\langle i j \mid r_{k}\right\rangle$ e o vetor $u_{a}^{*}$ tem componentes $u_{a_{i}}^{*}=\left\langle u_{a} \mid i\right\rangle$, onde $\left|u_{a}\right\rangle$ é um estado pertencente a $C_{a}^{n}$. Na seção 3.1, relacionamos a eq. (3.4) com um problema de autovalores para encontrarmos os estados com posto de Schmidt menor que $n$ em $\mathcal{P}_{|\psi\rangle}$. No caso da eq. (3.20), o melhor que podemos fazer é escrever

$$
\operatorname{det}\left[\sum_{k} \alpha_{k} R_{k}\right]=0
$$

Desta eq. (3.21), podemos deduzir que existe pelo menos uma superfície $(n-1)$ dimensional contida em $\mathcal{P}_{|\psi\rangle}$ constituída de estados com posto de Schmidt $(n-1)$ ou menor. Como o vínculo definido pela eq. (3.21) é não-linear, temos que essa superfície não é um plano e, portanto, existem $n$ estados linearmente independentes e com posto de Schmidt menor que $n$ que geram $\mathcal{P}_{|\psi\rangle}$. Isso significa que existem decomposições sub-Schmidt para qualquer estado emaranhado desta dimensionalidade com $n(n-1)$ estados produto.

Para verificarmos a existência de decomposições sub-Schmidt com menos estados produto, precisaríamos verificar a existência de um conjunto de $\alpha_{k}$ 's tais que todos os $(n-1)$ menores da matriz $\left(\sum_{k} \alpha_{k} R_{k}\right)$ se anulassem. Para o caso mais simples, com $n=3$, isso resulta em um sistema de nove polinômios em duas variáveis. Dessa forma, para sistemas mais gerais, é difícil escaparmos de um problema algébrico bastante complexo.

No entanto, sabemos que estados com decomposições sub-Schmidt com menos estados produto existem, pois podemos escrevê-los explicitamente. Como exemplo, podemos 
escrever um estado similar ao estado $|G H Z\rangle$ com dimensionalidade $(n, n, n)$

$$
\left|\psi_{G H Z}\right\rangle=\sum_{k} d_{k}\left|a_{k}\right\rangle\left|b_{k}\right\rangle\left|c_{k}\right\rangle
$$

onde $d_{k}$ são coeficientes complexos e $\left\{\left|a_{k}\right\rangle\right\},\left\{\left|b_{k}\right\rangle\right\}$ e $\left\{\left|c_{k}\right\rangle\right\}$ são estados linearmente independentes em $C_{a}^{n}, C_{b}^{n}$ e $C_{c}^{n}$, respectivamente. Particularmente, neste caso, podemos verificar que todos os estados que podem ser decompostos na forma (3.22) correspondem a uma classe SLOCC, pois sempre existem operadores lineares inversíveis, $A, B$ e $C$ em $n$ dimensões mapeando, respectivamente, $n$ vetores linearmente independentes $d_{k}\left|a_{k}\right\rangle,\left|b_{k}\right\rangle \mathrm{e}$ $\left|c_{k}\right\rangle$ em outros quaisquer $n$ vetores linearmente independentes $d_{k}^{\prime}\left|a_{k}^{\prime}\right\rangle,\left|b_{k}^{\prime}\right\rangle$ e $\left|c_{k}^{\prime}\right\rangle$. Portanto é sempre possível satisfazermos a condição (2.3) do Teorema 2.1 para dois estados que podem ser decompostos na forma (3.22). 


\section{Conclusão}

Nós descrevemos um método para obtermos decomposições de estados emaranhados tri-partidos com um qubit, as quais envolvem um número de termos menor do que o obtido por sucessivas decomposições de Schmidt. Essas decomposições foram chamadas de decomposições sub-Schmidt. Esse método é útil para classificarmos muitos estados de acordo com as suas decomposições e também para estudarmos as correlações quânticas entre os subsistemas.

Além disso, obtivemos que estados com diferentes decomposições sub-Schmidt pertencem a diferentes classes de estados emaranhados por SLOCC. Também estabelecemos condições necessárias e suficientes para decidirmos se dois estados deste tipo pertencem a mesma classe SLOCC. E, no caso de pertencerem, somos capazes de determinar a operação do tipo SLOCC que transforma um estado no outro.

Por fim, também discutimos as dificuldades de generalizarmos o método desenvolvido. Apesar dessas dificuldades, nossos resultados devem contribuir para um entendimento maior do emaranhamento multi-partido. A interpretação das diferentes classes de estados emaranhados obtidas na forma de diagramas de correlações fornece novas perspectivas para o estudo do emaranhamento. Além disso, existe a possibilidade de utilizarmos essas correlações como uma forma de deteç̧ão desses estados em uma realização experimental dos mesmos. 


\section{Referências Bibliográficas}

[1] C. H. Bennett e S. J. Wiesner, Phys. Rev. Lett. 69, 2881 (1992).

[2] C. H. Bennett e S. J. Wiesner, Phys. Rev. Lett. 69, 2881 (1992). C. H. Bennett, G. Brassard, C. Crépeau, R. Jozsa, A. Peres e W. Wootters, Phys. Rev. Lett. 70, 1895 (1993).

[3] A. K. Ekert, Phys. Rev. Lett. 67, 661 (1991).

[4] M. A. Nielsen e I. L. Chuang, Quantum Computation and Quantum Information, Cambridge University Press (2000).

[5] J. Preskill, Lecture Notes for Physics 229: Quantum Information and Computation http://www.theory.caltech.edu/ preskill/ph229/.

[6] E. Schmidt, Math. Annalen, 63, 433 (1907). Veja também [10, 4] e J. M. Jauch, Foundations of Quantum Mechanics, Addison-Wesley (1968).

[7] E. Schrödinger, Proc. Camb. Phil. Soc. 31, 555 (1935).

[8] E. Schrödinger, Proc. Camb. Phil. Soc. 32, 446 (1936).

[9] H. Everett, Rev. Mod. Phys. 29, 454 (1957).

[10] J. von Neumann, Mathematical Foundations of Quantum Mechanics, Princeton University Press (1955).

[11] A. Einstein, B. Podolsky e N. Rosen, Phys. Rev 47, 777, (1935).

[12] J. A. Wheeler e W. H. Zurek, Quantum Theory and Measurement, Princeton University Press (1984).

[13] M. Nielsen, Phys. Rev. Lett. 83, 436 (1999).

[14] C. H. Bennett, H. J. Berstein, S. Popescu e B. Schumacher, Phys. Rev. A 53, 2046 (1996). 
[15] R. Horodecki, P. Horodecki, M. Horodecki e K. Horodecki, http://arxiv.org/abs/quant-ph/0702225 (2007).

[16] M. H. Partovi, Phys. Rev. Lett. 92, 077904 (2004).

[17] W Dür, G. Vidal e J. I. Cirac, Phys. Rev. A 62, 062314 (2000).

[18] C. H. Bennett, S. Popescu, D. Rohrlich, J. A. Smolin, e A. V. Thapliyal, Phys. Rev. A 63, 012307 (2000).

[19] D. Bohm, Quantum Theory, Academic Press (1951).

[20] J. S. Bell, Physics 1, 195-200 (1964). Republicado em Speakable and unspeakable in quantum mechanics, Cambridge University Press (1987).

[21] A. F. R. de Toledo Piza, www.fma.if.usp.br/ piza/ (2007).

[22] C. Lunkes, C. Brukner e V. Vedral, Phys. Rev. Lett. 95, 030503 (2005).

[23] L. Fu e J. Liu, Phys. Rev. A 74, 063614 (2006).

[24] H. Häffner, W Hänsel, C. F. Roos, J. Benhelm, D. Chek-al-kar, M. Chwalla, T. Körber, U. D. Rapol, M. Riebe, P. O. Schmidt, C. Becher, O. Gühne, W. Dür e R. Blatt, Nature 438, 643 (2005).

[25] C. Lu, X. Zhou, O. Gühne, W. Gao, J. Zhang, Z. Yuan, A. Goebel, T. Yang e J. Pan, Nature Physics 3, 91 (2007).

[26] D. M. Greenberg, M. A. Horne e A. Zeilinger, "Going beyond Bell's theorem", Bell's Theorem, Quantum Theory and Conceptions of the Universe, editado por M. Kafatos, Kluwer Academic (1989). Republicado em http://arxiv.org/abs/0712.0921.

[27] D. M. Greenberg, M. A. Horne, A. Shimony e A. Zeilinger, Am. J. Phys. 58, 1131 (1990).

[28] R. Raussendorf e H.J. Briegel, Phys. Rev. Lett. 86, 5188 (2001).

[29] R. Raussendorf, D. Browne e H.J. Briegel, Phys. Rev. A 68, 022312 (2003).

[30] F. Verstraete, J. Dehaene, B. De Moor e H. Verschelde, Phys. Rev. A 65, 052112 (2002).

[31] A. Miyake e F. Verstraete, Phys. Rev. A 69, 012101 (2004).

[32] D. Leibfried, E. Knill, S. Seidelin, J. Britton, R. B. Blakestad, J. Chiaverini, D. B. Hume, W. M. Itano, J. D. Jost, C. Langer, R. Ozeri, R. Reichle e D. J. Wineland, Nature 438, 639 (2005). 
[33] P. Walther, K. J. Resch, T. Rudolph, E. Schenck, H. Weinfurter, V. Vedral1, M. Aspelmeyer e A. Zeilinger, Nature 434, 169 (2005).

[34] W. K. Wooters e W. Zurek, Nature 299, 802 (1982).

[35] D. Dieks, Phys. Lett. A 92, 271 (1982).

[36] K. Kraus, Operations: Fundamentals Notions of Quantum Theory, Lectures notes on physics vol. 190, Springer-Verlag, Berlim (1983).

[37] K.-E. Hellwig e K. Kraus, Commun. Math. Phys. 11, 214 (1969).

[38] K.-E. Hellwig e K. Kraus, Commun. Math. Phys. 16, 142 (1970).

[39] M. F. Cornelio e A. F. R. de Toledo Piza, Phys. Rev. A 73, 032314 (2006).

[40] A. Miyake, Phys. Rev. A 67, 012108 (2003).

[41] L. Chen e Y. Chen, Phys. Rev. A 73, 052310 (2006).

[42] L. Chen, Y. Chen e Y. Mei, Phys. Rev. A 74, 052331 (2006).

[43] L. Lamata, J. León, D. Salgado, e E. Solano, Phys. Rev. A 74, 052336 (2006).

[44] L. Lamata, J. León, D. Salgado, e E. Solano, Phys. Rev. A 75, 022318 (2007).

[45] D. Li, X. Li, H. Huang e X. Li, Phys. Rev. A 76, 052311 (2007).

[46] A. Sanpera, R. Tarrach e G. Vidal, Phys. Rev. A 58, 826 (1998).

[47] R. A. Horn e C. R. Johnson, Matrix Analysis, Cambridge University Press (1985). 